\title{
Human Machine Interfaces for Teleoperators and Virtual Environments
}

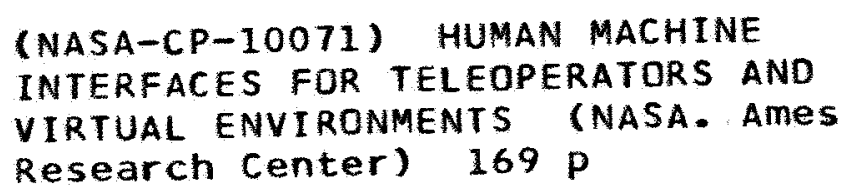

(NASA-CP-10071) HUMAN MACHINE INTERFACES FOR TELEOPERATORS AND Research (enter) $169 \mathrm{P}$

$63 / 54 \quad 0012870$

\author{
N95-14013 \\ --THRU-- \\ N95-14061 \\ Unclas
}

Summaries of papers from a conference held under the auspices of the Engineering Foundation and sponsored by NASA Ames Research Center, ONR, and IBM Corp., at. Santa Barbara, California, March 4-9, 1990 


\section{Human Machine Interfaces for Teleoperators and Virtual Environments}

Compiled by Nathaniel I. Durlach, Thomas B. Sheridan, and Stephen R. Ellis

Summaries of papers from a conference held under the auspices of the Engineering Foundation and sponsored by NASA Ames Research Center, ONR, and IBM Corp., at Santa Barbara, California, March 4-9, 1990

\section{NNSA}

National Aeronautics and Space Administration

Ames Research Center Moffett Field, California 94035-1000 



\section{PREFACE}

In the fall of 1989 we were approached by John Hollerbach, a member of the Engineering Conferences Advisory Committee of the Engineering Foundation, and asked if we would consider organizing a meeting around the general theme of teleoperation. After a number of discussions with a variety of people at MIT and elsewhere, we decided that it was time to bring together in an informal setting two research communities that are working on distinct but closely related types of systems: teleoperators and virtual environments.

Accordingly, we invited the best people available in these two emerging fields. We were extremely pleased that, with relatively few exceptions, all people contacted were enthusiastic about attending the proposed meeting.

We decided to have three kinds of sessions: invited-paper sessions, panel discussions, and poster sessions. The invited papers were divided into papers providing general background on sensorimotor performance and human-machine interfaces, papers on current problems and progress in teleoperation, and the same in virtual environments. Also, the panel discussions were subdivided into three problem areas: (1) telepresence and sensorimotor adaptation; (2) performance measurement and evaluation methods; and (3) design principles and predictive models. A mini seminar on teleoperators with micro slave robots was also included.

In order not to burden the people making presentations, we did not require preparation of written papers. Accordingly, this report in no sense constitutes a proceedings. It is, rather, a collection of conference-related fragments, highly non-uniform and totally unedited, that we hope will give people, particularly those who could not attend, a glimpse of the potential of these fields and how they interplay. We have included titles of presentations even when no written material was made available (either because no written material was submitted or because the presentation was withdrawn). Interested readers should contact the authors directly to obtain further information on these items. It is expected that a book on this topic, nourished by this conference, will be published in the not-too-distant future.

We are grateful to Dr. Melvin Montemerlo of NASA Headquarters, Dr. Michael Shafto of NASA Ames Research Center, Dr. Harold Hawkins of ONR, and Dr. Daniel Ling of IBM for their generous support of our meeting. We are also grateful to Dr. Stephen Ellis for his help in preparing this NASA publication.

Nat Durlach and Tom Sheridan

Conference Chairpersons

\section{PLimman PAGS marx NOT FHME}





\section{TABLE OF CONTENTS}

\section{Page}

PREFACE

iii

INTRODUCTION

$14 x$

BACKGROUND ON SENSORIMOTOR PERFORMANCE AND

HUMAN-MACHINE INTERFACES

3 ,

VISUAL INTERFACES: THE HUMAN PERCEIVER

5 Richard M. Held

AUDITORY INTERFACES: THE HUMAN PERCEIVER

$7-2$ H. Steven Colburn

TACTUAL INTERFACES: THE HUMAN PERCEIVER

9 M. A. Srinivasan

THE DISPLAY OF TACTILE INFORMATION

$11-4$

Carl E. Sherrick

NEURAL MECHANISMS OF KINESTHESIA

$13-5$

Francis J. Clark

ANALYSIS OF THE HUMAN OPERATOR SUBSYSTEMS

$15-6$ Lynette A. Jones and Ian W. Hunter

INTERSENSORY COORDINATION

17.7

J. R. Lackner

WATCH WHAT YOU SAY, YOUR COMPUTER MIGHT BE LISTENING:

A REVIEW OF AUTOMATED SPEECH RECOGNITION

$19-8$

Stephen De Gennaro

EYE MOVEMENT CONTROL AND TOP-DOWN SCANPATH VISION

AS A DESIGN METAPHOR FOR ROBOTIC VISION AND CONTROL Lawrence W. Stark

TELEOPERATORS

TELEROBOTIC REMOTE PRESENCE: ACHIEVEMENTS

AND CHALLENGES

Walter A. Aviles

ISSUES IN THE DESIGN OF HIGH DEXTERITY, FORCE REFLECTIVE TELEOPERATORS.

S. C. Jacobsen, E. K. Iversen, C. C. Davis, K. B. Biggers, and D. K. Backman 
TELE-EXISTENCE AND/OR CYBERNETIC INTERFACE

STUDIES IN JAPAN ..............................................................................................

Susumu Tachi

TELEROBOTICS IN THE DEEP OCEAN ................................................................ 36

Hagen Schempf and Dana R. Yoerger

MODELS OF REMOTE MANIPULATION IN SPACE .................................................. 37

Blake Hannaford 14

TELEOPERATING THE SPACE STATIONS............................................m 41 Marvin L. Minsky

SOME CRITERIA FOR TELEOPERATORS AND VIRTUAL ENVIRONMENTS

FROM EXPERIENCES WITH VEHICLE/OPERATOR SIMULATION

Henry R. Jex

MAN-MACHINE INTERFACES IN HEALTH CARE .................................................. 48

Steve Charles, M.D. and Roy E. Williams

TELEROBOTICS IN REHABILITATION: BARRIERS TO

A VIRTUAL EXISTENCE

Larry Leifer, Machiel Van Der Loos, and Stefan Michalowski

VIRTUAL ENVIRONMENTS

amil

CREATING BETTER VIRTUAL WORLDS

Thomas A. Furness, III

INTERACTING WITH VIRTUAL WORLDS David Zeltzer

VIRTUAL REALITY AT WORK

Frederick P. Brooks, Jr.

USING VIRTUAL REALITY AS A TOOL TO STUDY THE RELATIONSHIP

BETWEEN HUMAN BEINGS AND PHYSICAL REALITY ....................................

\section{J. Lanier}

THE AMES VIRTUAL ENVIRONMENT WORKSTATION:

IMPLEMENTATION ISSUES AND REQUIREMENTS

S. Fisher, R. Jacoby, S. Bryson, P. Stone, I. McDowall, M. Bolas,

D. Dasaro, E. Wenzel, C. Coler, and D. Kerr

VIRTUAL ACOUSTIC DISPLAYS Elizabeth M. Wenzel

VARIETIES OF VIRTUALIZATION .

Stephen R. Ellis 
THE EXPLORATION METAPHOR …................................................................... 81

Michael W. McGreevy 24

MEASUREMENT AND MODIFACTION OF THE EEG AND RELATED

BEHAVIOR

M. B. Sterman

THE "TELEPRASENZ” CONSORTIUM -STRUCTURE \& INTENTIONS ............... Jens Blauert

MACHINE PERCEPTION AND ITS ROLE IN VIRTUAL ENVIRONMENTS ......... 86 S. K. Ganapathy

SUMMARIES OF PANEL DISCUSSIONS.

TELEPRESENCE AND SENSORIMOTOR ADAPTATION

R. B. Welch, J. Loomis, R. Pepper, M. Kreuger, and T. Etter

PERFORMANCE MEASUREMENT AND EVALUATION METHODS $2 m$

Daniel T. Ling, Ruzena Bajcsy, Anatal Bejczy, Beth Marcus, and Misha Pavel

DESIGN PRINCIPLES AND PREDICTIVE MODELS H. L. Alexander, N. Hogan, J. M. Hollerback, I. Hunter, and W. Verplank

MINI SEMINAR: TELEOPERATORS WITH MICRO SLAVE ROBOTS

$$
\text { K. J. Gabriel, Chair }
$$

POSTER PRESENTATIONS.

THE HUMAN OPERATOR TRANSFER FUNCTION: IDENTIFICATION OF

THE LIMB MECHANICS SUBSYSTEM.

Lynette A. Jones and Ian W. Hunter

TACTILE DISCRIMINATION AND REPRESENTATIONS OF TEXTURE, SHAPE, AND SOFTNESS

M. A. Srinivasan and R. H. LaMotte

A HIGH PERFORMANCE TWO DEGREE-OF-FREEDOM KINESTHETIC INTERFACE

Bernard D. Adelstein and Michael J. Rosen

PERCEPTUAL AND PERFORMANCE CONSEQUENCES OF FLIGHT IN VIRTUAL WORLDS 
A DECADE OF TELEROBOTICS IN REHABILITATION: DEMONSTRATED UTILITY BLOCKED BY THE HIGH COST OF MANIPULATION AND THE COMPLEXITY OF THE USER INTERFACE

Larry Leifer, Stefan Michalowski, and Machiel Van Der Loos

A PHYSICAL CONTROL INTERFACE WITH PROPRIOCEPTIVE FEED-BACK AND MULTIPLE DEGREES OF FREEDOM.

G. H. Creasey, D. Gow, Y. Sloan, and B. Meadows

"ROTATIONAL WIND" INDICATOR ENHANCES CONTROL OF ROTATED

DISPLAYS

H. A. Cunningham and M. Pavel

OBJECTIVE EVALUATION OF SITUATION AWARENESS FOR DYNAMIC DECISION MAKERS IN TELEOPERATIONS Mica R. Endsley

DEVELOPMENT OF VISUAL 3D VIRTUAL ENVIRONMENT FOR CONTROL

SOFTWARE

Michitaka Hirose, Takeshi Myoi, Haruo Amari,

Kohei Inamura, and Lawrence Stark

HUMAN OPERATOR TRACKING PERFORMANCE WITH A VIBROTACTILE

DISPLAY

Gideon F. Inbar

IMPLEMENTATION ISSUES FOR A COMPACT 6 DEGREE OF FREEDOM FORCE REFLECTING HANDCONTROLLER WITH CUEING OF MODES

Heidi Jacobus, Alan J. Riggs, Charles Jacobus, and Yechiel Weinstein

ON THE ROLE OF EXCHANGE OF POWER AND INFORMATION

SIGNALS IN CONTROL AND STABILITY OF THE HUMAN-ROBOT

INTERACTION

H. KAZEROONI

A TWO DIMENSIONAL ARTIFICIAL REALITY

Myron W. Krueger

BINAURAL ROOM SIMULATION

ACTIVE LOCALIZATION OF VIRTUAL SOUNDS

HUMAN-MACHINE INTERFACE HARDWARE: THE NEXT DECADE 
COORDINATION OF HUMAN UPPER ARM AND FOREARM

MOTION IN RAPID EXTENSIONS

MANUAL DISCRIMINATION OF FORCE

X. D. Pang, H. Z. Tan, and N. I. Durlach

IMPLEMENTATION OF A THREE DEGREE OF FREEDOM, MOTOR/BRAKE HYBRID FORCE OUTPUT DEVICE FOR VIRTUAL ENVIRONMENT

CONTROL TASKS

Massimo Russo, Alfred Tadros, Professor Woodie Flowers, and

138

Professor David Zelter

HUMAN FACTORS REQUIREMENTS FOR TELEROBITIC COMMAND

AND CONTROL: THE EUROPEAN SPACE AGENCY EXPERIMENTAL

PROGRAMME

Robert J. Stone

VERDEX: A VIRTUAL ENVIRONMENT DEMONSTRATOR FOR

REMOTE DRIVING APPLICATIONS

Robert J. Stone

DESTABILIZING EFFECTS OF VISUAL ENVIRONMENT MOTIONS

SIMULATING EYE MOVEMENTS OR HEAD MOVEMENTS

K. D. White, D. Shuman, J. H. Krantz, C. B. Woods, and L. A. Kuntz

DESIGN WORK STATION FOR TELEOPERATION TASK ANALYSIS

K. Corker

SUPER AUDITORY LOCALIZATION FOR HUMAN MACHINE

INTERFACES

N. I. Durlach

CURRENT RESEARCH ON COCKPIT APPLICATIONS OF 3-D

AUDITORY DISPLAYS

K. O'Donnel and W. T. Melton 


\section{INTRODUCTION}

In a teleoperator system (see the accompanying figure), the human operator senses, moves within, and operates upon a remote or hazardous environment by means of a slave mechanism (a mechanism often referred to as a teleoperator). Signals in this environment are sensed by devices on the slave mechanism and communicated back to a human-machine interface and displayed to the human operator; the human operator's responses are sensed by devices at the interface and communicated back to the slave mechanism and used to control it. Teleoperators have been in use since the first nuclear laboratory experiments in the late 1940's. Teleoperation in the deep ocean, in space, in mines, and other remote, often hazardous, environments soon followed. Applications in medicine, involving exploration and operation within the human body, are now receiving increased attention. Early teleoperators were directly controlled by the operator in position (using isomorphic masterslave manipulators) or in rate (where joystick position determined manipulator or vehicle rate). More recently, computers have intervened, and the operator has become a supervisor, issuing subtask goal and procedural commands and letting the teleoperator's own computer then implement those instructions by closing a control loop through its own sensors and actuators. We term this more sophisticated teleoperator a telerobot and the mode of control supervisory control. General discussions of teleoperation are available in Johnsen and Corliss, 1971; Vertut and Coiffet, 1986; and Sheridan, 1989.

In a virtual-environment system (again see the accompanying figure), the interactive humanmachine interface is retained but the slave mechanism and its environment are replaced by a computer simulation. Video is replaced by computer graphics. The auditory and force sensations imparted to the human operator are similarly computer-generated. Since the rules governing the behavior of the simulation are under the control of the system designer, the operator in such systems can be presented with totally artificial worlds (e.g., having new physical laws). In contrast to a teleoperator system, where the purpose is to extend the operator's sensorimotor system in a manner that facilitates exploration and manipulation of the physical environment, in a virtual-environment system, the purpose is to train, inform, alter, or study the human operator or to modify the state of the computer and the information environment. A major application in which the human operator is the target (because the goal is training) is that of flight simulation. Although flight simulators have been around for more than a decade, they had little impact outside aviation presumably because the application was so specialized and so expensive. Clearly, what has created the recent flurry of interest in virtual environments is the great reduction in cost and increase in power of computer-graphic workstations, augmented, of course by miniaturization of sensors and display devices. Now some of the main applications are occurring in the entertainment industry (e.g., video games). Soon, we expect, there will be an increasing emphasis on designing virtual-environment systems specifically for scientific study of sensorimotor performance and for behavior modification by biofeedback. Applications directed more towards changing the information environment than the human operator involve use of the system for scientific visualization and theoretical modelling (as in work on molecular docking). Also, virtual environment systems are now being incorporated within teleoperator systems in cases for which planning modes or predictive model outputs are required. The idea in this case is for the operator to steer the teleoperator through simulated operations presented computergraphically just before performing the real operations. Alternatively, computer-graphic predictions of trajectory (bases on past and present control inputs) can be displayed automatically while the 
operator flies the vehicle or guides the manipulator. Such model-based predictions can then be superposed on the teleoperation video. General background on virtual-environments can be found in Sutherland, 1965; Krueger, 1983; Bolt, 1984; Haber, 1986; Foley, 1987; Fisher et al., 1988; Chung et al., 1989; Lanier, 1990; and Brooks et al., 1990.

In either a teleoperator or virtual-environment system, when the sensory feedback can be made sufficiently compelling (through the use of multimodality displays in which the output is influenced by the motor behavior of the human operator) to make the operator feel present at the remote or imaginary site, the system is said to have telepresence. The extent to which telepresence is important for achieving good performance is itself an interesting research topic.

In both teleoperator and virtual-environment systems, the human operator is effectively transformed into a new creature that is present in a new interactive environment. Also, in both cases the characteristics of the proposed transformation, as well as the sense of presence (or telepresence) in the new environment, are strongly influenced by the human-machine interface. Although there are some significant differences in the interface issues associated with the two types of systems (for example, in a teleoperator system it may be impossible to code certain targets independently because of inadequate resolution in the sensory system of the slave mechanism), there is clearly much in common. It is these common elements, namely, the transformed presence and the human-machine interface, that constitute the primary focus of this conference.

Nat Durlach and Tom Sheridan Conference Chairpersons

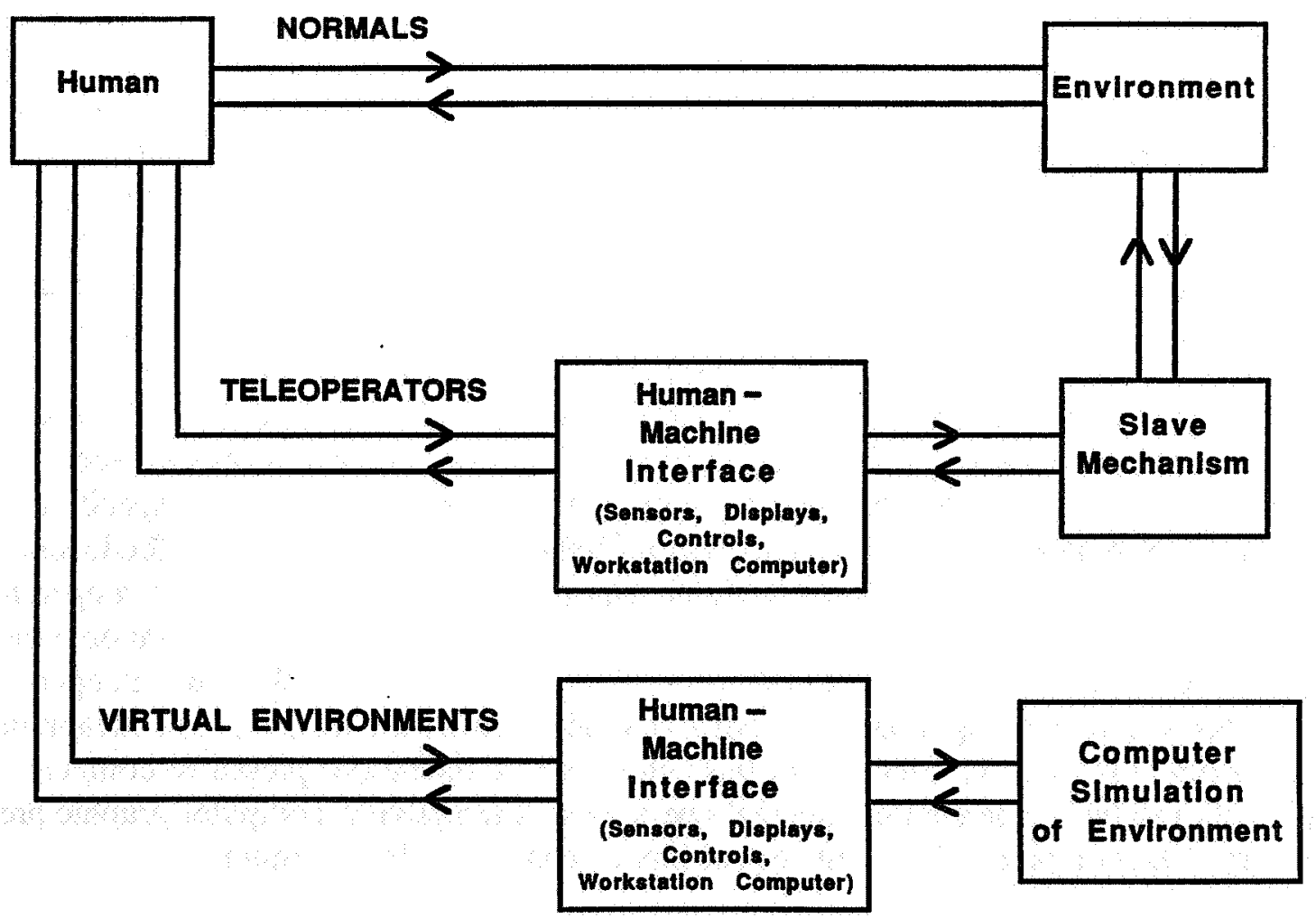




\section{BACKGROUND ON SENSORIMOTOR PERFORMANCE AND HUMAN-MACHINE INTERFACES}





\section{VISUAL INTERFACES: THE HUMAN PERCEIVER}

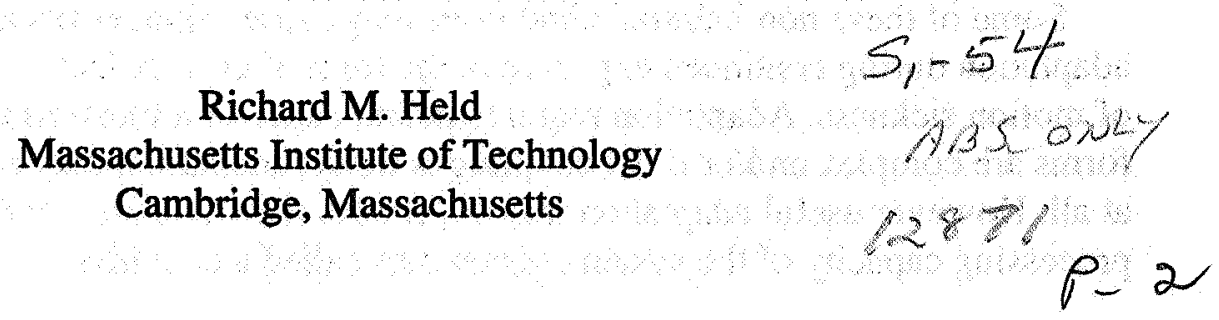

I was asked to speak about how knowledge of visual perception could be applied to achieve teleoperation and virtual worlds. After a little thought I decided that reversing subject and object makes the question more interesting and provocative. The practice of teleoperation and virtual world technology challenges our understanding of perception, and in the following I try to explain what I mean. But first let me say that vision science incorporates a vast amount of useful knowledge about the capabilities of the visual system. Fortunately, most of it is well-codified in the handbooks, of which the most recent and complete is Boff, Kaufman, and Thomas's Handbook of Visual Perception and Performance (1989). Unfortunately, this codified knowledge does not prepare us for the surprises attendant on the sensory and motor transformations effected by the new technologies. Instead, the surprises refer us to much less well understood areas. Hence the challenge to perceptual science from technology.

\section{World Regularities and the Habitual}

New technologies make it easy to transform spatially, temporally, and dynamically the relations among sensory inputs and motor outputs. For example, nothing could be simpler than making your hand appear to reach to the left when you intended it to reach to the right, to make you step up when you should step down, make the usually stable visible world appear to move as you move, and so on. The consequences can be errors in performance, disorientation, and a form of malaise like motion sickness. These examples make us realize to what extent our perceptions are dependent upon the regularities and habitual circumstances of our normal world and the dynamics of our actions in it. We are preadapted to these ordered states in multiple ways that are revealed by the effects of applying the new technologies to the human-machine interface. Compared to our knowledge of basic visual processes, our understanding of these orderings is limited but not insignificant. Some of it has been relegated to the fringes of the field as aberrant and bizarre forms of perception. The following table enumerates several types of transformation and examples.

\begin{tabular}{ll}
\multicolumn{1}{c}{ Type } & \multicolumn{1}{c}{ Examples } \\
Spatial Incongruity & $\begin{array}{l}\text { Japanese Illusion } \\
\text { Sensory Rearrangement }\end{array}$ \\
Temporal Asynchrony & $\begin{array}{l}\text { Phase Shift } \\
\text { Time Delay }\end{array}$ \\
& $\begin{array}{l}\text { Microgravity } \\
\text { Force Amplification }\end{array}$ \\
Altered Dynamics & Blank Visual Field \\
Deprivation & High Noise Level
\end{tabular}


Some of these non-habitual conditions may cause initial errors and disorientations. Some show adaptation during continued exposure in the form of error reduction, better orientation, and reduction of motion sickness. Adaptation requires maintenance of a transformed set of regularities. If the transforms are complex and/or if the coupling is noisy, adaptation may be either very slow or not possible at all. However useful adaptation may be, it does take time and represents an additional load on the processing capacity of the system, sometimes called a cognitive load.

\section{Telepresence and Presence}

A particularly intriguing problem, related to the above-mentioned considerations, is the creation of telepresence. It raises the question of what conditions specify the sense of presence - a question that seems at first blush so obvious as to require no thought. It is the first axiom of perceiving, namely, that the observer be there to perceive. It implies a here and now spatial juxtaposition (confrontation) of object and observer. We can begin to identify conditions for the sense of presence as:

1. Independent perception of an observer and an object external to the observer.

2. Covariation of sensed observer movement independent of the sensed object yields the sense of an object external to the observer.

3. Observer has access to the sensed object at will.

Achievement of these conditions is, in turn, related to the regularities and habitual circumstances discussed above. 


\title{
AUDITORY INTERFACES: THE HUMAN PERCEIVER
}

\author{
H. Steven Colburn \\ Boston University \\ Boston, Massachusetts
}

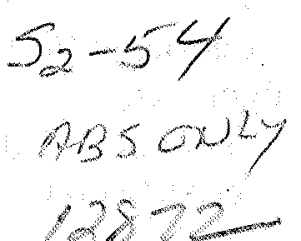

This talk is a brief introduction to the basic auditory abilities of the human perceiver with particular attention toward issues that may be important for the design of auditory interfaces. The importance of appropriate auditory inputs to observers with normal hearing is probably related to the role of hearing as an omnidirectional, early warning system and to its role as the primary vehicle for communication of strong personal feelings.

Several basic properties of the human auditory perceiver should be kept in mind when designing interfaces. The range and resolution of the perceiver is impressive in terms of frequency (three decades with sensitivity to changes of $0.2 \%$ ) and intensity (110 decibels with sensitivity to changes of a fraction of a decibel at almost all levels) so that the standards for clean sounds are quite high. On the other hand, our memory for absolute stimuli is much more limited, particularly with respect to intensity. In an identification paradigm, perceptual systems are generally limited to about $7 \pm 2$ intensity levels, so that the ability to identify is much more limited than the ability to differentiate. The notion of the critical bandwidth is also important in understanding auditory phenomena and abilities. In its crudest form, the critical band notion, which is consistent with a wide variety of data, is that the auditory system behaves as if it contained peripheral filters with bandwidths of about onetenth of an octave.

The issues that arise in the design of auditory interfaces are different according to whether the interface is an isomorphic representation of the acoustic environment with natural sounds and transformations or a non-isomorphic mapping from fundamentally different inputs. In the completely isomorphic case, the problem is conceptually straightforward but very difficult to realize. Although one simply has to recreate the appropriate acoustic stimuli (from a robot or from a simulation) at the ears of the human listener, there are substantial engineering challenges in reproducing or creating the appropriate sounds, In addition to the obvious requirements for excellent fidelity in the acoustic system, the echo and reverberation effects on each sound must be appropriate and, critically, the motion of the listener's head must be coupled to the robot or program. The complexity of the problem can be illustrated by thinking of the effect on the sounds received at the ears by several sound sources in a normal environment. In this case, as the head moves, the "head-related transfer function" of each source changes in a way that can only be specified with knowledge of the position of the source and the position and orientation of the head. The processing of the human perceiver imposes important constraints on the quality of the reproduced sound signal.

In the simplest non-isomorphic cases, such as a scaled robot or a normal-sized robot in an environment with acoustic properties different than air (e.g., a helium rich environment), there are different problems, including the translations of signals to signals appropriate for human listeners. For example, a very small robot would have very small interaural differences that could be essentially undetectable to the human perceiver with an associated loss of ability to judge the azimuth of a 
sound source. If these signals are processed to provide an appropriate stimulus on a human scale, the influence of the environment on the signals would have to be transformed and this would require significant knowledge of an environment that may be unfamiliar to the robot or its controller. The transformation of the received stimuli to provide appropriate acoustic inputs for a natural (human sized) situation requires a relatively full knowledge of the sound field.

In the case of more extreme deviations from a humanoid robot or simulation, the issues become conceptually difficult to think about. For example, if the robot has multiple ears so that a more sophisticated picture of the acoustic environment could be computed by the interface, the mapping of the signals or the information to the two ears of the human perceiver is difficult to design. Either some of the information will be neglected or we must learn how to increase the presentation of information to the human, possible by parsing the acoustic field and presenting multiple acoustic objects. This is a higher level recoding problem, and depends on techniques that have not yet been developed. Some of the attributes that result in the perception of separate acoustic objects are known and include separate spatial location, consistent modulation envelopes in frequency and amplitude, consistent harmonic structure, and others.

In some cases, if a stimulus is to be perceived as being generated by the listener, then there are special problems due to the fact that the sound is being received over two pathways, by the air-conduction pathway and by the bone-conduction pathway. The air-conducted sound can be monitored and transformed appropriately by the interface, but the bone-conduction pathway is difficult to cancel so that the stimulus perceived by the subject is a combination of the bone-conducted sound and the air-conducted sound provided by the interface. Learning would be a problem here if the new environment was trying to simulate an environment with light or heavy gases for example. In this case, the auditory stimulus would be a combination of the higher-pitched sound and the normalpitched sound.

There are many cases in which unnatural stimuli are more effective than naturally occurring stimuli. [The argument that the human sensori-motor system is optimized by evolution (often applied to speech signals) cannot be applied in many cases because the constraints of the system are so complex and even difficult to know. In addition to questions of acoustics, for example, one can ask how important is the eating function to the design of the mouth or the necessity of being born to the size of the head?] When there is a well-defined task to be performed, it is often advantageous to provide non-natural processing of the stimuli. For example, angle resolution near the midline can be improved significantly by presenting nonlinearly processed stimuli that would increase the interaural differences even though some distortion would be added that could be important for some stimuli. On the other hand, when the tasks are extremely varied or unpredictable, there are significant advantages to using natural stimuli and allowing the experience of the listener to be used to primary advantage with little training required for many tasks. In addition, there are situations in which an unnatural stimulus may be advantageous such as the design of hearing aids with expanded ranges of levels and frequencies or in the design of auditory displays for non-auditory information. 


\title{
TACTUAL INTERFACES: THE HUMAN PERCEIVER
}

\author{
M. A. Srinivasan \\ Massachusetts Institute of Technology \\ Cambridge, Massachusetts
}

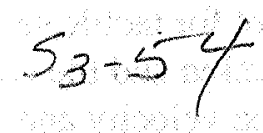

Increasingly complex human-machine interactions, such as in teleoperation or in virtual envi- $P-2$ ronments, have necessitated the optimal use of the human tactual channel for information transfer. This need leads to a demand for a basic understanding of how the human tactual system works, so that the tactual interface between the human and the machine can receive the command signals from the human, as well as display the information to the human, in a manner that appears natural to the human. The tactual information consists of two components: (1) contact information which specifies the nature of direct contact with the object; (2) kinesthetic information which refers to the position and motion of the limbs. In this talk, we shall mostly be concerned with contact information.

A wide variety of tactile perceptions that we distinguish can be thought of as combinations of two classes of "primitives": mode of contact (static indentation, relative tangential motion or slip, and vibration) and object properties (surface microtexture, shape, and compliance). It is plausible that by understanding the peripheral and central processing involved in each pairwise combination among the primitive classes, the processing of more complex natural stimuli can be unravelled. Our knowledge of cutaneous information processing is mostly based on human psychophysics and electrophysiological recordings from monkey afferent fibers. These fibers are of four types: Two types of slowly adapting fibers (SA I and II) that are associated with Merkel cells and Ruffini endings, and are responsive both when an object in contact is moving against the skin as well as under steady indentations; Two types of rapidly adapting fibers which respond only when the skin is moving, one type (RAs) terminating in Meissner corpuscles, and the other (PCs) in Pacinian corpuscles. When a probe indenting the skin is vibrated, lowest response threshold amplitudes for SAs are at frequencies of 0-10 Hz, for RAs at 20-50 Hz, and for PCs at 100-300 Hz. Summarized below are some of the known results on the detection of slip and microtexture, shape, and compliance.

Humans cannot detect the slip of a smooth glass plate on the skin; existence of detectable features on the surface is necessary. However, surprisingly small features (for example, 2 microns high dot causes RAs to respond and 0.06 microns high grating causes PCs to respond) on smooth surfaces are detected by humans and lead to the detection of slip of these surfaces, with the geometry of the microfeatures governing the associated neural codes. The division of labor among the different types of fiber populations in signaling the different events on the skin is clear-cut: SAs signal the direction of skin stretch and hence the direction of impending slip; RAs and PCs signal the occurrence of slip with spatiotemporal or intensive codes, depending on whether the microfeature is a local one on a smooth background, or is distributed on the surface, respectively. When the surface features are of sizes greater than the response thresholds of all the receptors, redundant spatiotemporal and intensive information from all three afferent fiber types is available for the detection of slip.

Among the different possible geometric representations of the shape of objects, the intrinsic description, i.e., the surface curvature as a function of the distance along the surface, seems to be 
relevant for tactile sensing. Recordings of afferent responses to diverse shapes show that the depth of indentation and the change in curvature of the skin surface are represented in SA responses; in addition, the velocity and the rate of change in skin surface curvature are represented in both SA and RA responses. The primary reason for such neural encoding is the form of the spatial variation of the pressure imposed by the object on the skin surface: Pressure peaks occur where the depths of indentation and/or changes in the skin surface curvature are high. The skin effectively acts as a low-pass filter in transmitting the mechanical signals, and the mechanoreceptors respond to the blurred versions of the surface pressure distribution, thus encoding the shape of the object in terms of its surface curvatures.

Compliant objects can be of two types: (1) those with a rigid surface (such as a piano key); (2) those with a deformable surface (such as cheese). By conducting psychophysical experiments under both active and passive touch, with or without local anesthesia that blocked the contact information, the following conclusions were reached. Humans are very good at discriminating softness of objects with deformable surfaces, and the discrimination is based on contact information. For compliant objects with rigid surfaces, discrimination of compliance is reliable only under active touch, when both contact and kinesthetic information is available, and the discriminability is poorer than that for objects with deformable surfaces. Preliminary neurophysiological data show that mostly SA and to a lesser extent RA responses provide the basis for our ability to discriminate compliance. 


\section{THE DISPLAY OF TACTILE INFORMATION}

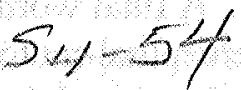

Carl E. Sherrick

Princeton University

Princeton, New Jersey

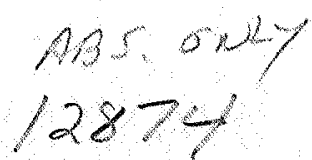

There are a number of examples of natural tactile displays that can give us some insights about the solid geometry of touch, and recent experimental work on the subject has extended our thinking considerably. The concern of this conference is, however, more with synthetic or artificial displays for the production of a virtual environment. The majority of the talk will dwell on the features of synthetic displays that have enjoyed some success in one of two enterprises: The study of the spatiotemporal dimensions of stimuli that afford accurate and rapid processing of environmental information, or the use of displays in the design of sensory aids for disabled persons.

Examples of synthetic displays are given, with some discussion of their application, success, and reasons for their failure, i.e., their current disuse. In the past, displays have been mainly of the type called diffuse, i.e., spread over a large area of the body. In more recent years, the improvements in electronic technology have made possible the production of more and more dense arrays of tactile stimulators, some of which, like the Optacon of Telesensory Systems, Inc., were developed for applied purposes, and now are used in basic research as well.

This conference is best served by my talk if I list some of the things that have been learned about the spatio-temporal properties of tactile displays that could be useful in the design of displays for teleoperator systems. One of these has to do with the site of the display, which is commonly of concern to sensory aids developers for reasons of comfort and appearance. In addition to such concerns, however, it has become clear that issues of efficiency of energy usage, related to the thresholds for feeling, and the needed dynamic range of such devices, are also important to the designer, and these parameters vary greatly with the site of stimulation. Of equal importance is spatial acuity, i.e., the discrimination of high spatial frequencies on the skin, which varies systematically with the location of the stimulus array.

There is a considerable literature dealing with such space-time phenomena as masking, real and apparent movement on the skin, temporal integration of both energy and coded information, saltation, and lateralization. These will be discussed in some detail as useful boundary conditions for device design.

Another class of phenomena exist that may appear at first to be too esoteric for applied consideration, but which, because they have only a short history of systematic research, deserve consideration for their potential in enriching the perceptual field of the tactile display. One of these is the interaction of touch with other modalities, and a good example is found in studies of the temperature sense. Another is the phenomenon of distal attribution, which involves the transference of the display properties from the veridical site of stimulation to the object being simulated by the display. 
A final word will be said about the importance of the learning process in acquiring a little-used perceptual-motor skill, which teleoperator systems must demand of the user when an unusual source such as touch is incorporated into the information stream. 


\title{
NEURAL MECHANISMS OF KINESTHESIA
}

\author{
Francis J. Clark \\ University of Nebraska \\ Omaha, Nebraska
}

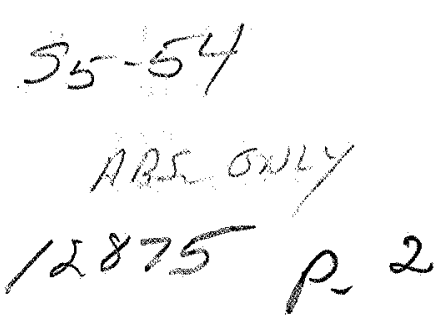

Our primary interest is in where the signals for position and movement of the limbs originate and how the information is encoded. Humans can independently sense the positions and movements of their limbs in the absence of vision, and these senses are derived from mechanoreceptors located in the limbs. Receptors in muscles, skin and joints are potential sources of position and movement signals, but how (and whether) each of these groups contributes to kinesthesia remains uncertain. Recent evidence indicates that a sense of static-position derives from length receptors in muscle, whereas receptors in the skin, and possible though not likely the joints, can signal movement of a limb but not its static position.

We distinguish this static-position sense from a movement sense on the basis of whether a subject's ability to sense a small displacement ( 3 to $5 \mathrm{deg}$ ) of a joint varies with the rate of joint displacement. Therefore, if subjects possess an awareness of the static-position of a joint, slow rates of rotation should not seriously degrade their ability to sense displacements. In the absence of a staticposition sense, subjects would rely on movement signals that do depend on the rate of rotation and their ability to detect displacements should decrement with decreasing rate. This is roughly analogous to distinguishing a low-pass filter (a static-position sense) from a high-pass filter ) a movement sense only) by their frequency response characteristics.

The interphalangeal (IP) joints of the fingers (and probably the toes) appear to lack a static-position sense. Subjects were unable to detect large displacements of the IP joints if the displacements were slow enough (our tests used rates less than $1 \mathrm{deg} / \mathrm{min}$ ). In contrast, small displacements of the metacarpophalangeal (MCP) joint of the finger could be detected at the slowest rates we used. This is a surprising finding given that people seem quite unaware they have this kinesthetic deficit at the fingers.

At present, we are examining the consequences of not having a static-position sense at the fingers apart from an inability to detect very slow displacements. Using a more demanding test, we have found no substantial difference in the accuracy of reproducing target positions passively imposed on the MCP joint and the proximal interphalangeal (PIP) joint of the index finger (the MCP has and the PIP lacks a static-position sense). Our working hypothesis is that subjects normally use movement sense mechanisms to detect displacements, probably because they are faster, but how (or whether) subjects manage to obtain an absolute position reference with only a movement sense available remains unknown and is an issue under investigation.

We have also shown that subjects can reproduce target positions of a joint from memory about as accurately as they can match the position of the joint to its corresponding joint on the opposite limb. Reproducing targets from memory remains remarkably accurate even after 24 hours. The existence of a stable, long-term memory for limb position is an important component of a model for motor 
control we are investigating. Irrespective of the mechanism, the accuracy of this memory complicates the study of position and movement senses. For example, one cannot study a static-position sense by moving a joint to some position and waiting until all movement signals have faded away. With a memory, one could not distinguish a true static-position mechanism from a mechanism that derived position from a velocity signal and stored the result in a memory. 


\title{
ANALYSIS OF THE HUMAN OPERATOR SUBSYSTEMS
}

$$
56-5 y
$$

\author{
Lynette A. Jones and Ian W. Hunter \\ McGill University \\ Montreal, Quebec, CANADA
}

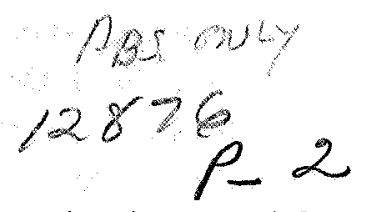

Except in low-bandwidth systems, knowledge of the human operator transfer function is essential for high-performance telerobotic systems.. This information has usually been derived from detailed analyses of tracking performance, in which the human operator is considered as a complete system rather than as a summation of a number of subsystems, each of which influences the operator's output. Limitations in analytic techniques and insufficient knowledge about the characteristics of the human operator have resulted in these "black box" depictions of tracking performance. One consequence of this approach is that it has been impossible to determine the source of change in the parameters of the transfer function under different conditions. Studies of one of these subsystems, the limb mechanics system, demonstrate that large parameter variations can occur (e.g., the elastic stiffness of a limb can change over a 50-fold range for different levels of muscle activation), that can have a profound effect on the stability of force-reflecting telerobot systems.

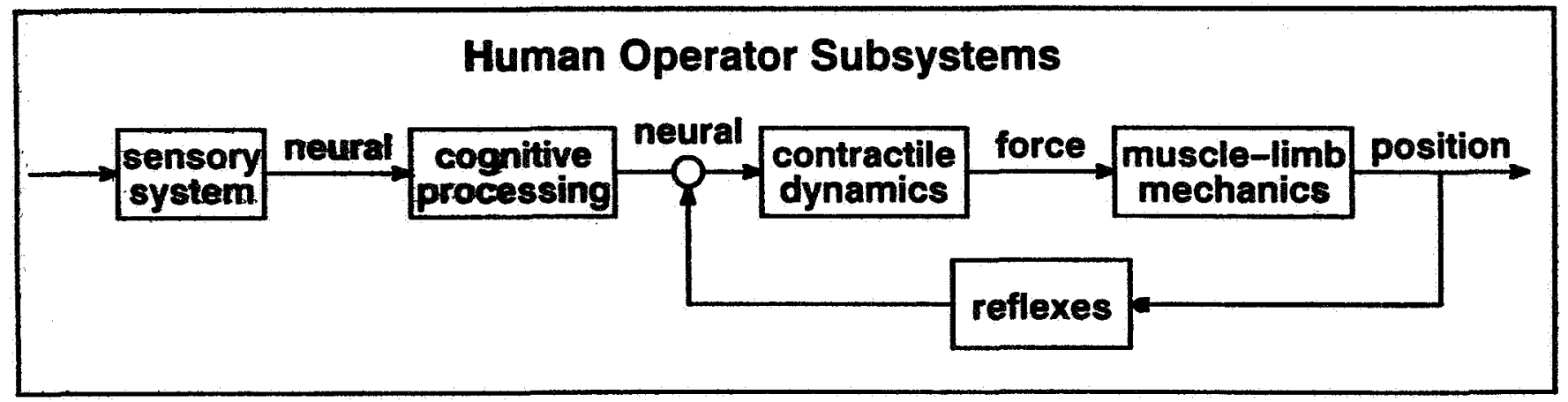

One of the objectives of our research is to decompose the performance of the human operator system (i.e., its damping, natural frequency, pure delay, static gain) in terms of the subsystems depicted above, in order to establish how the dynamics of each of these elements influence the operator's responses. For example, the delays associated with each subsystem are different (ranging from $15 \mathrm{~ms}$ for contractile dynamics to over $150 \mathrm{~ms}$ for cognitive processing), and for some subsystems the transformations are linear and time invariant, whereas for others they are nonlinear and time varying. Recent advances in nonlinear system identification make it possible to characterize the dynamics of the human operator subsystems in a single experimental session. Ideally the same equipment and analytic procedures should be used in all phases of this process. On the basis of the results from such an analysis, it should be possible to predict for any given operator the conditions (e.g., muscle-joint segment, type of controlled response, mechanical properties of the human interface) under which control is optimal. 
With this objective in mind, we have constructed an apparatus for studying the human neuromuscular system in terms of its neural (contractile dynamics), mechanical (limb mechanics), perceptual (sensory thresholds), and tracking (human interface) characteristics. The apparatus consists of two powerful computer-controlled linear actuators instrumented with displacement and force transducers. With this equipment the neuromuscular function of the muscles controlling the elbow can be determined by imposing small, wide-bandwidth, stochastic length perturbations to the forearm. In addition the mechanical, reflex, and neural excitation to force dynamics of the neuromuscular system controlling the elbow flexor/extensor muscles can be measured, sensory thresholds can be calculated, and finally the tracking dynamics of the human operator control system can be determined. To date, studies of these subsystems have been limited to single joints such as the elbow, and the effects on tracking performance of varying the response type and human-interface properties have been investigated.

The characteristics of the human operator in a pursuit-tracking task change as a function of the operator's response. When subjects track a visually presented target either by changing the position of their forearm or by modulating the forces generated by the elbow flexor and extensor muscles, force control is superior to position control in terms of response delay (100 ms less), but subjects are more accurate when position is the controlled variable. Position control is, however, influenced by the mechanical properties of the human interface, and in particular changes in manipulator stiffness affect both response delay (less at higher [up to $2000 \mathrm{~N} / \mathrm{m}$ ] stiffness amplitudes) and tracking accuracy (more accurate at lower $[<1500 \mathrm{~N} / \mathrm{m}]$ amplitudes). 


\section{INTERSENSORY COORDINATION}

\author{
J. R. Lackner \\ Brandeis University \\ Waltham, Massachusetts
}

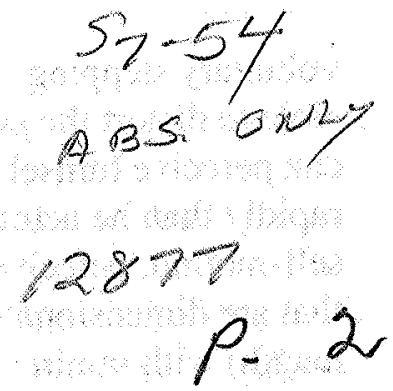

Perception of body orientation and configuration is dependent on multiple sources of afferent and efferent information about the spatial configuration of the body and its relationship to the support and force characteristics of the surroundings. Such information allows us under normal circumstances to preserve an accurate and stable distinction between those changes in receptor activity contingent on self-motion and those contingent on motion of or within the environment. Maintenance of this distinction is essential for the ongoing control of normal body movement and posture.

Because of this active interrelating of sensory and motor cues whenever there is an error in the registration of ongoing body orientation, auditory and visual mislocalizations of related magnitude and time course can occur. As a consequence, depending on the perceived spatial representation of the body, identical patterns of arrival time and intensity cues at the ears (and identical retinal and oculomotor signals) can give rise to the perception of sounds (and visual objects) in widely disparate spatial positions in relation to the head and to the external environment. The interaction between sensory localization and the representation of body orientation is actually bidirectional. For example, a rotating sound field can elicit illusory self-rotation and compensatory eye movements in a stationary listener.

The conscious appreciation of limb position is influenced by muscle afferent signals. When the body or tendon of a muscle is mechanically vibrated, for example at $120 \mathrm{~Hz}$, the muscle reflexly contracts. This contraction is.known as a tonic vibration reflex (TVR). If the motion of a limb moving under the action of a TVR is resisted, illusory motion of the (unseen) stationary limb will be experienced. For example, if the biceps brachii muscle is vibrated, the restrained forearm will be felt to move into extension. A target light attached to the stationary index finger of the hand, in an otherwise dark room, will be seen to move in keeping with the apparent motion of the arm even though the eyes maintain steady fixation. If the subject is allowed to see his index finger in relation to the surroundings, but not the rest of his forearm, he will see his finger in one spatial location and feel the rest of his arm to be in another spatially distinct location.

During body movement in a high force field, the relationship between muscle activity and muscle spindle feedback is altered, much as in the case of the vibration illusions just described. If an individual does deep knee bends during exposure to twice earth gravity, then he will misperceive the extent of his own voluntary motion and simultaneously experience visual motion of his surroundings by the amount of that misperception. This demonstrates that there is a dynamic relationship between intended movements and the actual movements executed, and the perception of those movements.

During normal terrestrial locomotion, deformation of the optic projection of the visual world, the intensity and timing of efferent signals and of muscle afferent signals, and the distribution of contact forces of support on the body co-vary and are mutually corroborative. If the relationship between 
voluntary stepping movements, body displacement, and visual flow are artificially altered, it is possible to distort the perception of voluntary actions so that an individual voluntarily stepping forward can perceive himself to be voluntarily walking backwards, or to be stepping more slowly or more rapidly than he actually is. Common to all the remappings elicited are combinations of perceived self-motion, perceived limb activity, seen motion of the visual world, and felt motion of the ground that are dimensionally consistent (even if not physically probable, such as changes in apparent leg length) with minimal departures from terrestrial constraints. 


\title{
WATCH WHAT YOU SAY, YOUR COMPUTER MIGHT BE LISTENING: A REVIEW OF AUTOMATED SPEECH RECOGNITION
}

\author{
Stephen De Gennaro \\ IBM Thomas J. Watson Research Center \\ Yorktown Heights, New York
}

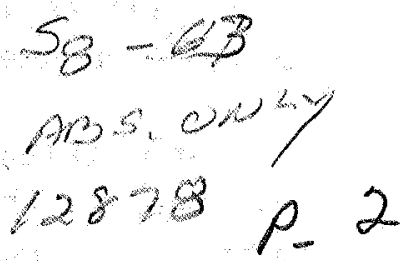

Spoken language is the most convenient and natural means by which people interact with each other and is, therefore, a promising candidate for human-machine interactions. Speech also offers an additional channel for hands-busy applications, complementing the use of motor output channels for control.

Current speech recognition systems vary considerably across a number of important characteristics, including vocabulary size, speaking mode, training requirements for new speakers, robustness to acoustic environments, and accuracy. Algorithmically, these systems range from rule-based techniques through more probabilistic or self-learning approaches such as Hidden Markov Modeling and Neural Networks.

This tutorial begins with a brief summary of the relevant features of current speech recognition systems and the strengths and weaknesses of the various algorithmic approaches.

Issues critical to the successful application of speech recognition in human-machine interactions will be discussed in more detail. These issues include: "raw" recognition vs. speech understanding, grammar-based, constrained dialog vs.. natural-language, free discourse and the imposition of appropriate constraints to make the task technologically feasible, with acceptable accuracy. The potential delay in recognition, due to algorithmic features or to interactive verification of recognized words for error correction, must also be considered. The need for verification is determined by both the expected error rate and the cost of each error. The cost function could be dynamic if errors will have significantly different impact given the state of the system.

As in any human-machine interface, not all of the constraints are due to the "-machine" half of the interaction; the "human-" partner also imposes a share of burdens. For example, people will not always say exactly what is expected of them, even when carefully prompted with a small set of possible choices. Stress, attention, and general physical state can have strong effects on system performance.

For applications that are not currently speech-driven, it might also be necessary to reformulate the basic human-machine interface. For example, moving a cursor over a document by voice in the most straightforward way (up-up-up-left-left- ...) is not as efficient (and probably not as acceptable) as moving the cursor by keys or mouse. Recasting the basic problem as "go to the word HELLO is the second paragraph" provides a better match between the task and speech control. Navigating a robot by voice instead of by joystick might require similar reformulation. Of course, while "go to the word ..." is more natural, it imposes new requirements to understand the command and map it into the appropriate action. 
Given these characteristics, it is not yet reasonable to expect that an off-the-shelf speech recognizer can be used as a transparent replacement for a keyboard or joystick as input to any arbitrary application. The highest performance levels will be achieved when the application and recognition processes are more tightly coupled.

Using existing speech recognition technology, it is certainly possible to build successful virtual environments. This is particularly true for systems that mimic environments in which speech is already the primary communication mode, and where the control "protocols" have been well defined. For example, Air Traffic Control training consoles have been demonstrated using continuous speech, but the task is constrained to deal only with objects (i.e., specific flights) on the trainee's console to provide reasonable accuracy and response delay. A general problem with coupling, however, is that it leaves the system vulnerable to unanticipated scenarios, with concomitant risk that the system will not respond gracefully to "exceptional" interactions.

This tutorial will conclude with some pointers to conferences and publications that reflect the current state of commercially available speech recognizers, and the state of research in this field. 


\section{EYE MOVEMENT CONTROL AND TOP-DOWN SCANPATH VISION AS A DESIGN METAPHOR FOR ROBOTIC VISION AND CONTROL}

\section{Eye Movement Control}

\author{
Lawrence W. Stark \\ University of California \\ Berkeley, California
}

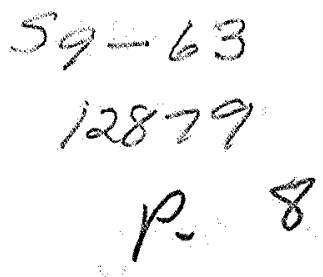

\section{Kinetics: Time Optimal Control of Saccades}

Eye movements are elegant biological examples for application of time-optimal engineering control theory. The muscles that drive eyeball rotations are very long and strong and fast. The eyeball has low rotational inertia so that the internal viscosity of active muscle dominates plant dynamics; thus we may think of the system as a visco-elastic load. What controller signal shapes would produce a time-optimal movement? First, reciprocal innervation, proposed by Descartes (\#149) and experimentally found by Sherrington, should be observed (Figure 1A). Next, a set of multipulse-step control signals first described by Clark and Stark (\#137) can be justified on theoretical grounds by Bellman-Pontriagin bang-bang theory.

\section{Dual Mode Control: Sampled Data Operator}

Another beautiful example is the sampled data control model of Young and Stark (\#27) that was experimentally verified by study of transient responses in normal subjects. A further exciting demonstration was found in the explanation of "macro-saccadic oscillation" found in patients with cerebellar disease that effectively removed the continual adaptive gain adjustment and left the patient with high gain instability oscillation (\#156). (Figure 1B).

\section{Higher Level EM Control and Scanpath Theory}

\section{Active Looking and the Kantian Theory of Perception}

A more metaphoric theory, the Scanpath Theory has been developed by Noton and Stark $(\# 110)$ and Stark and Ellis (\#211) to account for the repetitive sequence of saccades that enable the eye and its fovea centralis to traverse over important sub-features of a picture or scene. This has been taken to mean than an internal cognitive model drives active looking in a top-down manner. The evidence to support this theory has to do with statistical proof that there is indeed repetition of scanpath sequences. Further evidence comes from studies of ambiguous figures wherein the same subject looking at the same picture switches both mental states of perception and patterns of repetitive saccadic sequences. Most recently, Brandt and Stark (in preparation) have used visualization tasks. A subject recalling a previously seen diagram demonstrates scanpath movements similar to those looking at the earlier viewed diagram; here only the internal model can be responsible for the scanpath sequence and, indeed, string editing distances support this finding quantitatively. (Figure 2A). 
The philosopher, Kant, reasoned that internal representations (Platonic ideals) controlled active perception in a top-down procedure that could then organize sensation (without time and without space) received from the chaotic work of appearances. In this view, the virtual model within a human brain is more real than the external world of the naive realistic! (Figure 2B).

Acknowledgment to: NASA Ames Research Center Cooperative Agreement NCC-286, Dr. Stephen Ellis, Technical Monitor, and to California Institute of Technology, Jet Propulsion Laboratories, Dr. Anatal Bejczy, JPL Technical Manager, for their support. 


\section{Top-Down Robotic Vision}

\section{Models of Robots and ROIs}

For our use of visual feedback, for use by autonomous algorithms, for use by manual operators, and for use by supervisory controllers. We have taken this top-down scheme from human vision and introduced it as a basic design principle in our Telerobotic Laboratory. Instead of searching for important features of the TRWE (telerobotic working environment) with bottom-up image processing algorithms, we instead use a top-down approach. We already know where a particular robotic link is located in 3D space, we know where the cameras are pointed, we know what important

parameters we need to measure. Thus, we use our knowledge in the form of forward-loop control model not only to control the robots and the cameras, but also to direct the image processing (\#312 and A304). We construct ROIs (regions-of-interest) for each camera frame so that the image processing can be done rapidly and robustly (Figure $3 \mathrm{~A}$ ).

Within the ROIs, local thresholding and centroid calculations enable us to compute a feedback model, indicated by crosses that represent the visual measurement of the location of key on-thescene-visual-enhancements. This, of course, is done dynamically so that rapid feedback permits closed-loop control of the robotic movement, with the human operator acting as a supervisory controller only (Figure 3B).

\section{Over-All Robotic Control Scheme}

\section{Model of RWE}

The overall model (Figure 4A) permits one to appreciate the communication functions of our top-down image processing scheme. Communication is restricted to parameters of the visual models, an enormous bandwidth reduction compared to sending video pictures of a number of views of the TRWE. The supervisory controller looks not at the camera view of the scene, but rather at a model of the TRWE! Is this going to be satisfactory? (\#T411, \#313).

\section{Evaluation of Displays and HMD}

Several evaluation studies of visual display requirements have been carried out in our laboratory (\#301, \#A313). Dr. Won Soo Kim and colleagues have established that, although for some purposes enhanced 2D displays enable the operator to perform satisfactorily, virtual 3D displays are robust to various deficiencies and enable performance at least as good as the best $2 \mathrm{D}$ displays. Also, models alone or models superimposed onto video pictures are better than video pictures for shortterm robotic control tasks (Figure 4C, \#A291). In another study, Dr. Greg Tharp and colleagues demonstrated that 5 to $15 \mathrm{~Hz}$ update rates for a stereo display were fast enough to permit the subject to slew his head freely without suffering a penalty in performance (delays were less than 1 frame interval). (Figure 4B). 


\section{Summary}

Telerobotics with virtual environments is an exciting and worthwhile field of research. It offers challenges to engineering design of displays, of control, and of communication schemes. It relates to human performance since the human operator is a component either as a direct manual controller or as a supervisory controller. It will teach us more about human capacities and we can use knowledge of human plans and schemas to produce initial designs. We are also learning more about ourselves. Did we thing about "presence" before we considered telepresence? 


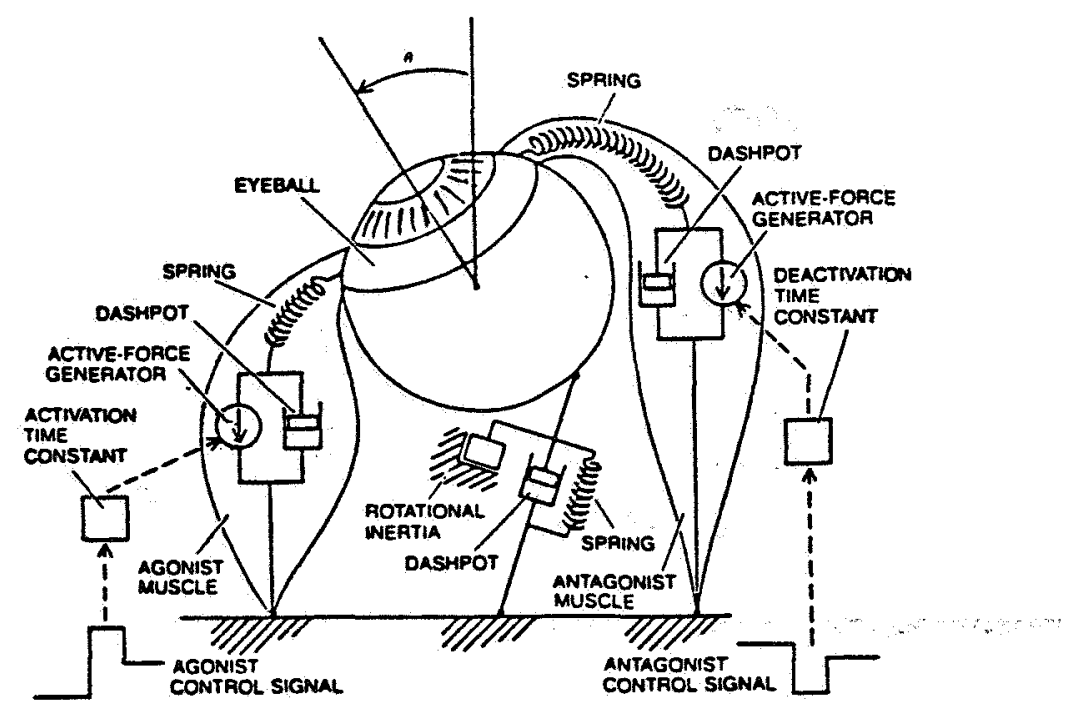

Figure 1A. Descartes model.

\#149 Kenneth J. Ciuffreda and Lawrence Stark, “Descartes' Law of Reciprocal Innervation," American Journal of Optometry and Physiological Optics 52: 663-673 (1975)
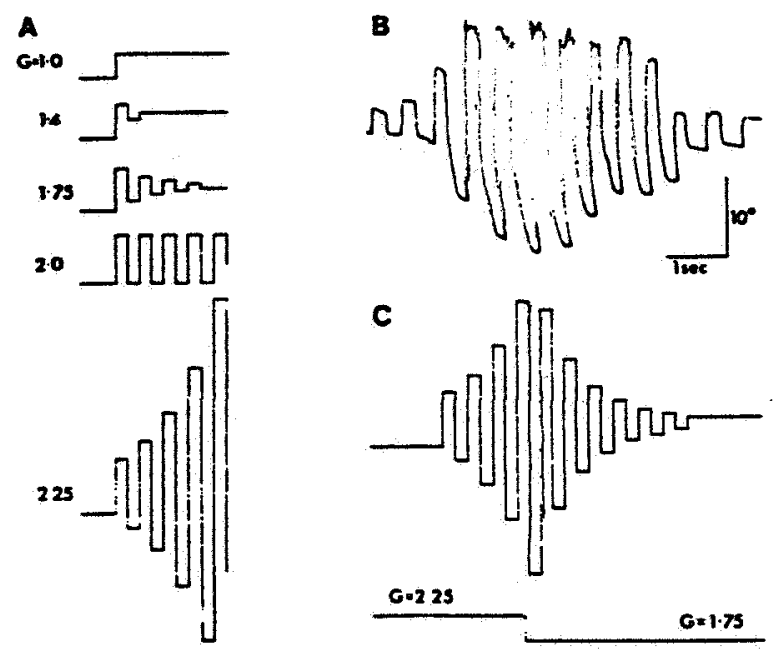

Figure 1B. SD clinical simulation.

\#27 Laurance R. Young and Lawrence Stark, "Variable Feedback Experiments Testing a Sampled Data Model for Eye Tracking Movements." IEEE Transactions on Human Factors in Electronics HFE-4: 38-51 (1963)

\#156 John B. Selhourst, Lawrence Stark, Alfred L. Ochs and William F. Hoyt, "Disorders in Cerebellar Ocular Motor Control. II. Macrosaccadic Oscillation: An Oculugraph, Control System and Clinico-Anatomical Analysis.” Brain 99: 509-522 (1976). 


\section{SCANPATH THEORY}
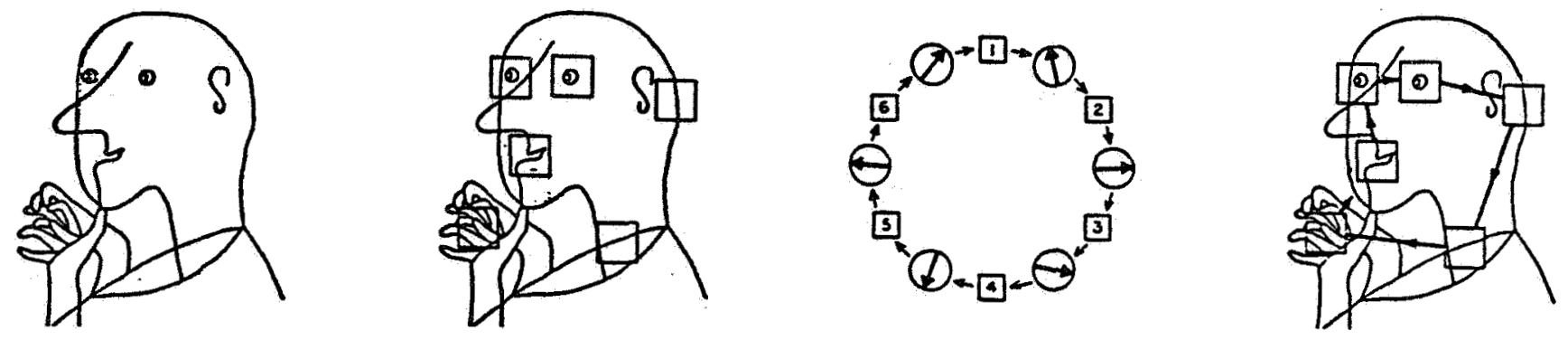

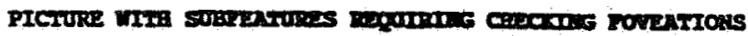

COGIIIVE MODEL CONrROLIING ACIIVE LOOKING

Figure 2A. Scanpath theory.

\#1 10 David Noton and Lawrence Stark, "Eye Movements and Visual Perception." Scientific American 224: 334-43 (1971).

\#211 Lawrence Stark and Stephen Ellis, "Scanpaths Revisited: Cognitive Models Direct Active Looking." In: Eye Movements, Cognition and Visual Perception, ed. Fisher, Monty and Senders, New Jersey: Eribaum Press, 193-226 (1981).

\section{KANTIAN DEFINITIONS}

1
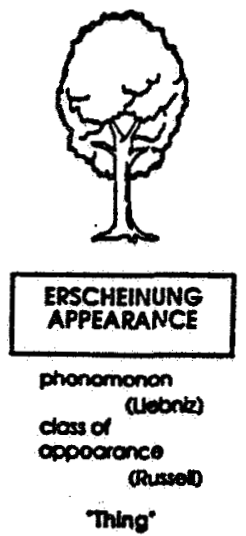

2
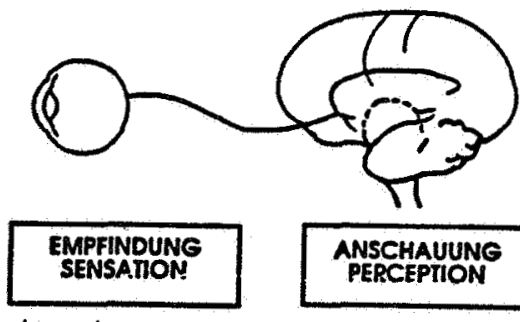

impresuion

doctrine of spectis nerve ending

"physlologr whout
3

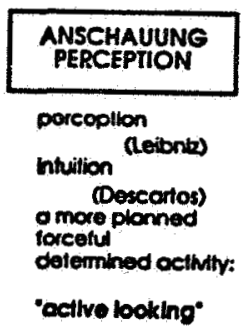

4
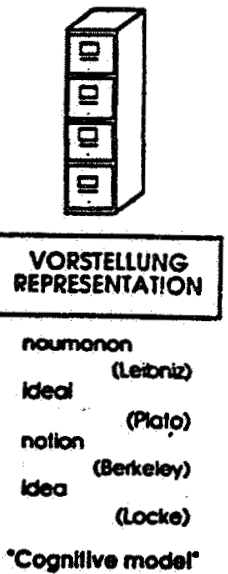

Figure 2B.

\#287 L. Stark, W.H. Zangmeister, B. Hannaford and K. Kunze, "Use of Models of Brainstem Reflexes for Clinical Research." In: Clinical Problems of Brainstem Disorders; Thieme Publishers, New York: 172-184 (1986). 

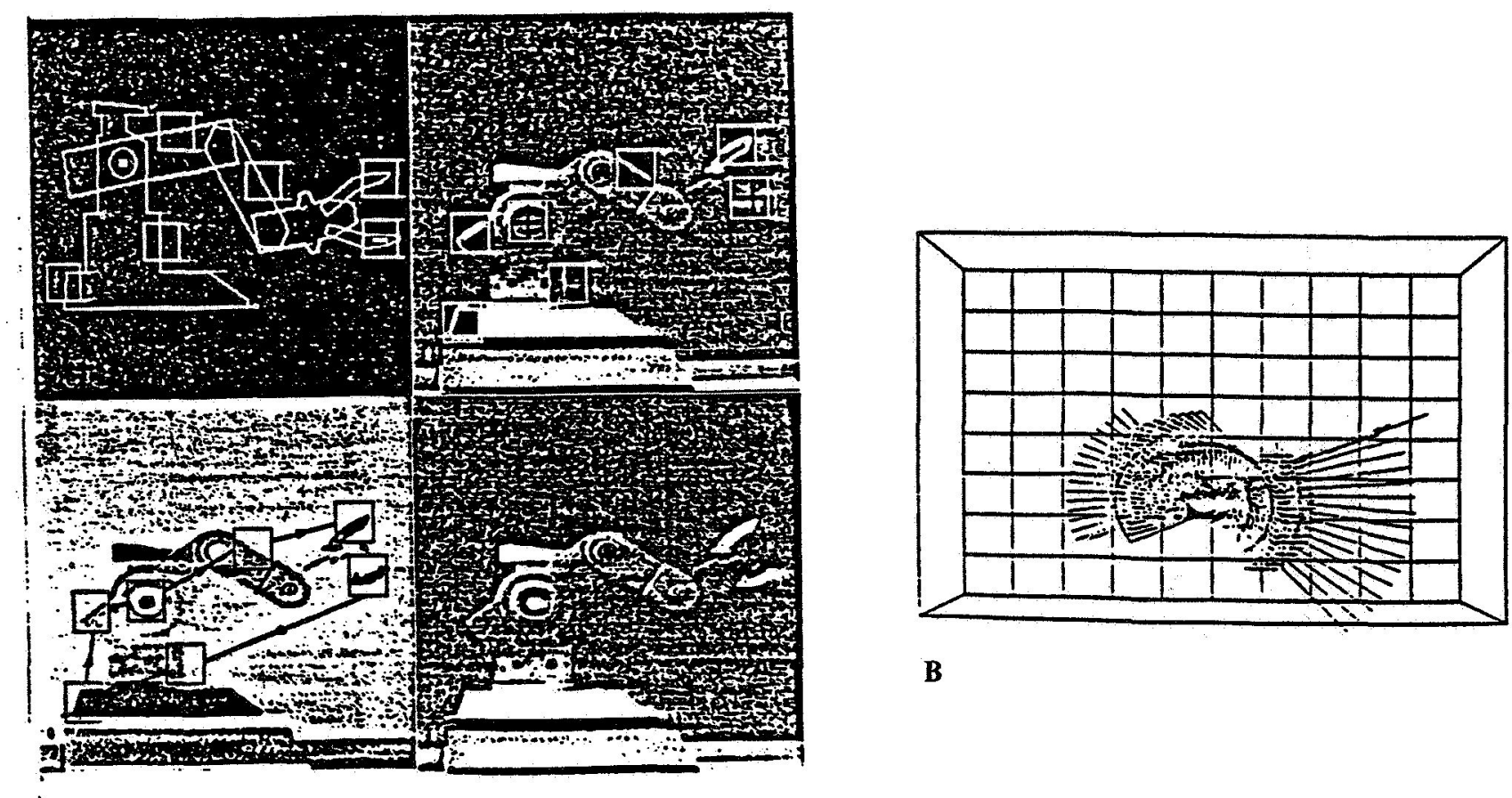

B

\section{A}

Figure $3 \mathrm{~A}$ and $3 \mathrm{~B}$.

\#312 Lawrence Stark, Barbara Mills, An Nguyen, Huy X. Ngo, "Instrumentation and Robotic Image Processing Using Top-Down Model Control." Robotics and Manufacturing, Jamshidi et al., eds., ASME, NY (1988): 675-682).

\#A304 An Nguyen and Lawrence Stark, "3D Model Control of Image Processing." NASA Conference of Space Telerobotics, Pasadena (1989).

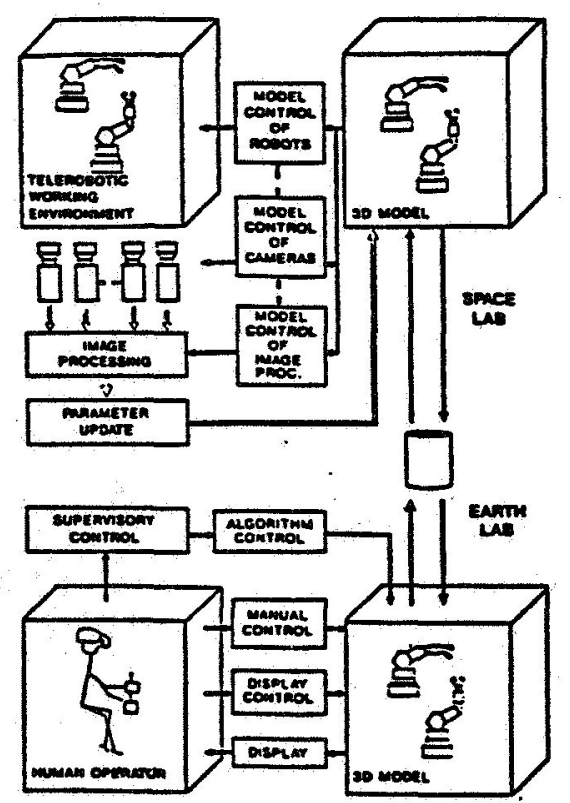

Figure 4A.

\#313 Lawrence Stark, et al., "Telerobotics: Problems and Research Needs." IEEE Transactions, Aerospace and Electrical Systems 24: 542-551 (1988).

\#T411 Lawrence Stark, “Biological Redundancy,” in progress. 

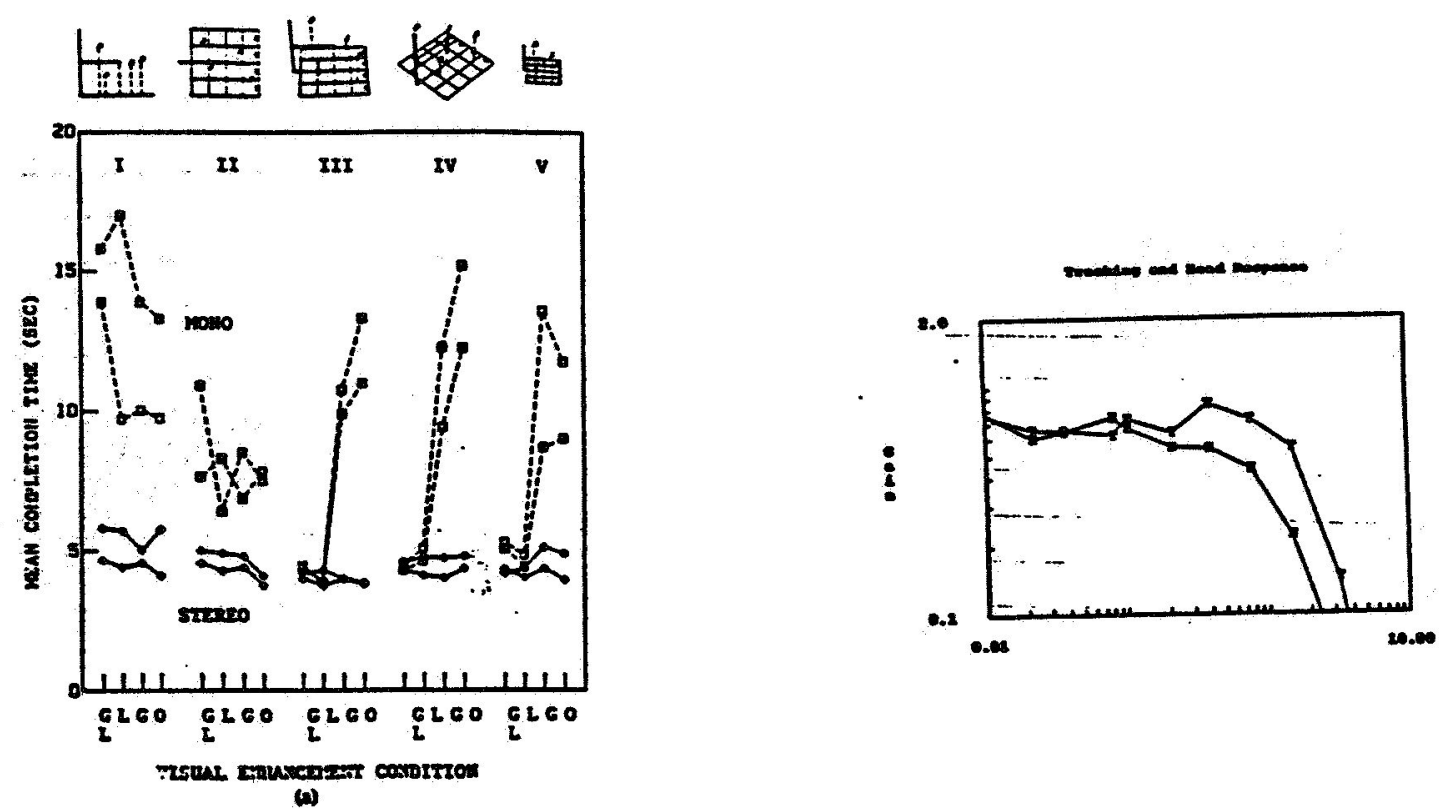

Figure 4B.

\#301 Won Soo Kim, Frank Tendrick, Stephen Ellis and Lawrence Stark, "Visual Enhancement in Pick-and-Place Tasks: Human Operators Controlling a Simulated Cylindrical Manipulator." IEEE Journal of Robotics and Automation RA-3: 426-436 (1987).

\#A313 Gregory Tharp, Andrew Liu, Hitomi Yamashita, Lawrence Stark, Brenda Wong and Jurgen Dee, "A Helmet Mounted Display to Adapt the Telerobotic Environment to Human Vision." Johnson Space Center, Texas (1989).
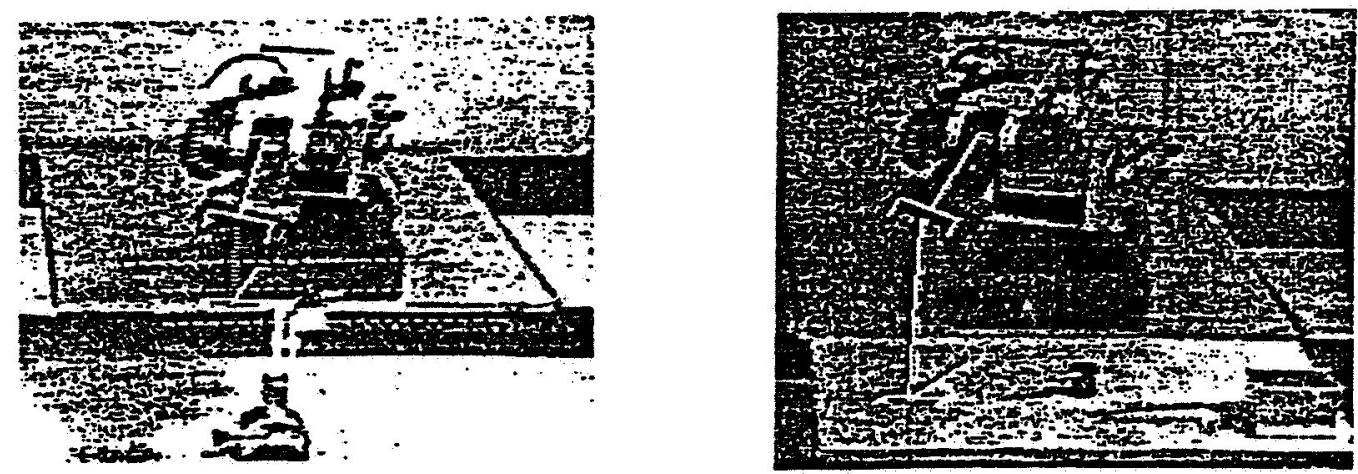

Figure 4C.

\#A291 Kim, W. S., Takeda, M. and Stark, L., “On-the-screen Visual Enhancements for a Telerobotics Vision System." Proceedings IEEE International Conference, Systems, Man \& Cybernetics, Beijing (1988). 


\section{TELEOPERATORS}





\title{
TELEROBOTIC REMOTE PRESENCE: ACHIEVEMENTS AND
}

\section{CHALLENGES}

\author{
$5,0-37$ \\ Walter A. Aviles \\ Naval Ocean Systems Center \\ Kailua, Hawaii

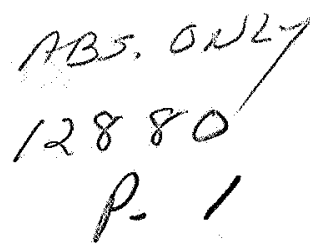

The Marine Sciences and Technology Department of the Naval Ocean Systems Center has as one of its particular thrusts the development of telerobotic work systems which blend the capabilities of a human with those afforded by automation technologies. The goal of these systems is to give a human operator the subjective experience of being at a remote work site when he is in fact at a location well removed from the (potentially hazardous) work area. Systems of this type are said to exhibit "telepresence". Telepresence is accomplished through the use of spatially correspondent controls and high-fidelity sensory feedback systems which utilize the human operator's cognitive and sensory capabilities in a manner that is natural to him. A major advantage of the telepresence approach is that the human operator does not have to concentrate on how to use unfamiliar or unnatural displays and controls but instead uses his own cognitive and manipulative abilities to perform the task. As a consequence, the telepresence approach minimizes the time required to perform work as well as minimizing the amount of training required to use sophisticated undersea work systems.

Although the goals of a telerobotic human-machine interface exhibiting telepresence are intuitively straightforward to grasp, the quantification of terms such as "correspondent controls" and "high-fidelity sensory feedback" in a manner useful for development remains a major challenge to the field. In addition, the proper blending of automated assistance, remote work site sensory feedback, and artificially generated sensory feedback, is both a fascinating and challenging endeavor. This talk will attempt to outline the progress in human-machine interfaces from the perspective of telerobotic remote presence and to suggest avenues for future research. 


\title{
ISSUES IN THE DESIGN OF HIGH DEXTERITY, FORCE REFLECTIVE TELEOPERATORS
}

\author{
S. C. Jacobsen, E. K. Iversen, C. C. Davis, K. B. Biggers, and D. K. Backman \\ University of Utah \\ Salt Lake City, Utah
}

The Center for Engineering Design at the University of Utah is developing an anthropomorphic, hydraulically actuated, teleoperated arm. The system includes a sixteen degree-of-freedom slave manipulator controlled by a kinematically identical, sixteen degree-of-freedom force-reflective, exoskeletal master. The project has focused on four areas: (a) formulating a realistic set of design objectives which balance, against technical realities, the desire for performance, reliability and economy, (b) understanding control issues, (c) designing and fabricating new subsystems (presently unavailable) necessary for the construction of a successful machine and (d) integrating subsystems, through a series of prototype stages, into an operational teleoperation system.

Generating a comprehensive set of design objectives required the consideration of fundamental questions such as: (1) what should be the kinematic configuration of master and slave, hand and arm, (2) how many degrees of freedom will be necessary for the level of dexterity desired, (3) should the master be exoskeletal, or some other configuration, (4) what is the importance of force reflection and how can its fidelity be improved beyond current systems, (5) what are the realistic actuation alternatives considering active and passive performance requirements, (6) what performance is required of sensor systems, (7) when necessary, can tendons be successfully used to remotize actuators.

Our work in the control area focused on: (1) developing stable position and torque feedback approaches which result in desirable inter-system impedance (frequency dependent gain, stiffness and damping), (2) generating acceptable joint-torque compensation which removes undesirable effects of gravity, mass and viscous drag, (3) increasing system bandwidth to improve operator awareness of loads imposed on the slave.

Extensive efforts were expended in the design and fabrication of presently unavailable subsystems such as: (1) rotary and linear hydraulic actuators along with required servo valves, (2) jointtorque sensors capable of remote-axis-sensing, high stiffness, low stiction and minimal sensing of loads on other axes, (3) position sensors with acceptable accuracy, (4) computation systems which include digital and analog electronics for implementation of servo, intermediate and higher level control functions, (5) systems for long range communication between the master and slave systems. Designing subsystems such that they "package" into the available shaped volumes as well as satisfying other constraints was a problem of substantial magnitude. Packaging remains an underestimated problem which in fact dominates many design decisions.

Finally, work is proceeding towards integrating subsystems into a working machine which actually achieves its objectives with respect to performance, reliability and economy. The system must: (1) be capable of operating in sea environments which includes corrosion and substantial 
pressure, (2) survive substantial mechanical abuse and misuse, (3) include mechanical machineoperator interfaces which insure comfortable, nonfatiguing operation over long periods of time.

The presentation will address the issues mentioned above as well as include a video demonstration of the system executing selected dextrous tasks. 


\title{
TELE-EXISTENCE AND/OR CYBERNETIC INTERFACE STUDIES
} IN JAPAN

\author{
Susumu Tachi \\ Mechanical Engineering Laboratory, MITI \\ Tsukuba Science City, Ibaraki, JAPAN
}

Tele-existence aims at a natural and efficient remote control of robots by providing the operator with a real time sensation of presence. It is an advanced type of teleoperation system which enables a human operator at the controls to perform remote manipulation tasks dexterously with the feeling that he or she exists in one of the remote anthropomorphic robots in the remote environment, e.g., in a hostile environment such as those of nuclear radiation, high temperature, and deep space.

Systematic research for the development of tele-existence has been conducted at MEL by feeding back rich sensory information which the remote robot has acquired to provide the operator with a real-time sensation of presence.

With the advent of science and technology, it has become possible to envision teleoperation with a sensation of presence. The concept of projecting ourselves by using robots, computers and cybernetic human interfaces is called Tele-Existence, and a lot of effort has been made for the realization of this concept. This concept also provides an extension of human sensing and muscular capabilities.

The tele-existence system consists of intelligent mobile robots, their supervisory subsystem, a remote-pressence subsystem and a sensory augmentation subsystem, which allows an operator to use the robot's ultrasonic, infrared and other, otherwise invisible, sensory information with the computer graphics-generated pseudo-realisitic sensation of presence. In the remote-presence subsystem, realistic visual, auditory, tactile, kinesthetic and vibratory displays will be realized (ref. 1).

Using this system, a human operator can be in a safe and comfortable environment and, at the same time, be present or exist at other environments where the robots are working. He or she will monitor the work through the robot's sensors, and, if necessary, conduct the task on behalf of the robot as if he was doing the work himself (ideally) or as if he was working inside the robot (práctically).

The principle of the tele-existence sensory display has been proposed. Its design procedure has been explicitly defined. Experimental visual display hardware has been built, and the feasibility of the visual display with the sensation of presence has been demonstrated by psychophysical experiments using the test hardware (refs. 1,2).

A method has also been proposed to develop a mobile tele-existence system, which can be remotely driven with the auditory and visual sensation of presence (ref. 3). A prototype mobile televehicle system has been constructed and the feasibility of the method has been evaluated. 
In order to study the use of the tele-existence system in the artificially constructed environment, the visual tele-existence simulator has been designed, a pseudo-real-time binocular solid model robot simulator has been made, and its feasibility has been experimentally evaluated (ref. 4).

An anthropomorphic robot mechanism with an arm having seven degrees of freedom has been designed and developed as a slave robot for feasibility experiments of teleoperation using the teleexistence method. An impedance controlled active display mechanism and a head mounted display have also been designed and developed as the display sub-system for the master. The robot's structural dimensions are set very close to those of humans, and it is designed to mimic the movement of humans (ref. 5). By using this tele-existence master slave system, the feasibility of the tele-existence master slave system has been demonstrated.

\section{REFERENCES}

1. S. Tachi et al., "Tele-existence (I) - Design and evaluation of a visual display with sensation of presence-," Proceedings of the 5th Symposium on Theory and Practice of Robots and Manipulators (RoManSy84), pp. 245-254, CISM-IFToMM, Udine, Italy, June 1984.

2. S. Tachi and H. Arai, "Study on tele-existence (II) - Three dimensional color display with sensation of presence - ," Proceedings of the "85 International Conference on Advanced Robotics (ICAR), pp. 345-352, Tokyo, Japan, Sept. 1985.

3. S. Tachi, H. Arai, I. Morimoto, and G. Seet, "Feasibility experiments on a mobile tele-existence system," The International Symposium and Exposition on Robots (19th ISIR), Sydney, Australia, November 1988.

4. S. Tachi, H, Arai, and T. Maeda, "Tele-existence simulator with artificial reality (1), - Design and evaluation of a binocular visual display using solid models-," Proceedings of the IEEE International Workshop on Intelligent Robots and Systems, pp. 719-724, 1988.

5. S. Tachi, H. Arai, and T. Maeda, "Development of an anthropomorphic tele-existence slave robot," Proceedings of the International Conference on Advanced Mechatronics (ICAM), pp. 385-390, Tokyo, Japan, May 1989. 


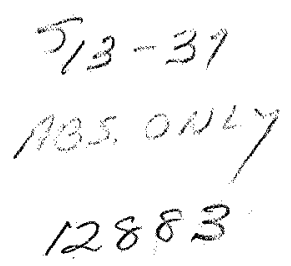

$p-1$

\section{TELEROBOTICS IN THE DEEP OCEAN}

\author{
Hagen Schempf and Dana R. Yoerger \\ Woods Hole Oceanographic Institution \\ Woods Hole, Massachusetts
}

Remotely operated vehicles can be made more useful for a wide range of survey tasks through the use of precision navigation and automatic control integrated under a proper supervisory control framework. Tasks ranging from scientific survey to ship hull inspection can be done more productively and produce a more useful result if the motions of the ROV are tightly controlled. A key benefit of automatic control is the ability to repeat a track at a later time to study dynamic processes seafloor.

This paper presents experimental results of the control system for the JASON ROV that has been designed for precision survey and other automated applications: The JASON control system emphasizes a form of supervisory control where the human pilot and the automatic system share the control task. Results presented included hovering, automatic track following, and several interactive modes.

JASON is equipped with an underwater manipulator that can exhibit a wide range of compliance through a combination of mechanical design and software control is described and its performance characterized. The major goal of the design was to produce a manipulator that can control the interaction forces with the work task and can work reliably in the hostile deep-ocean environment. The manipulator's performance has been characterized in the lab and its overall operational utility has been confirmed during an archaeological excavation at 700 meters depth in the Mediterranean. Results from the lab tests and sea trials will be presented. 


\title{
MODELS OF REMOTE MANIPULATION IN SPACE
}

\author{
Blake Hannaford \\ University of Washington \\ Seattle, Washington
}

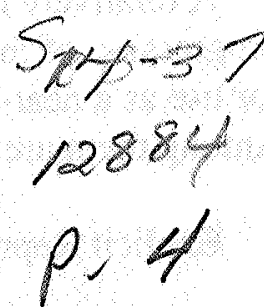

Astronauts operating in space can hardly be called autonomous. Their progress is constantly being monitored and supported by the ground team. The same procedures will apply to robotic systems in space - no matter how intelligent. This is also true of other important manipulation tasks. For example, a surgeon must perform an operation within a system of supervision and documented procedures which ensure quality care and accountability. What these two domains have in common is a high value environment in which a highly specialized manipulation is performed a small number of times (in contrast to factory automation). Thus robots involved in high value manipulation must be effectively coupled to a human operator either at the work-site or remotely connected via communication links.

Hundreds of hours of man in the loop experiments have been performed to quantify the performance of telemanipulation systems with different levels of capability.

In order to make use of experimental performance evaluation data, models must be developed. Powerful models of remote manipulation by humans can be used to predict manipulation performance in several ways. First, they will allow prediction of performance in future systems based on today's laboratory systems. In this paradigm, the models are developed from experimental data, and then used to predict performance in slightly different situations. Second, accurate telemanipulation models will allow design of manipulation systems which extend manipulation capability beyond its current bounds.

The barriers which are currently being extended by state of the art remote manipulation systems include distance and scale. Distance barriers are most relevant to space telemanipulation although the large manipulators made feasible by the lack of gravity involve scale factors of about 10 . Microteleoperators are now being developed which couple the operator to micro-worlds. For example, a force reflection system has recently been developed by IBM which couples the operator to the tip of a scanning tunneling microscope: a scale factor of about $10^{-7}$ !

The famous time delay problem inherent in overcoming the distance barrier is yielding to new methods in which control is shared between the manipulator and the human operator in ways which allow energetic interaction between manipulator and environment.

Models developed by several laboratories to explain the dynamics of kinesthetic force reflection will be briefly reviewed and applied to the experimental data. Two-port network models have been successful at improving the understanding and performance evaluation of teleoperators with kinesthetic force feedback in which the human operator, master manipulator, slave manipulator, and environment are coupled together through servo control loops. Such systems can create a sense that the operator directly manipulates the environment. 
A relatively new model of task performance based on Hidden Markov Models, is able to analyze progression of multi-stage compound tasks. The HMM has several properties that make it attractive for use as a near-real-time execution monitor and post hoc "explainer" in practical applications. Among them are:

- Explicit Representation of Uncertainty

- Support for "knowledge based" operation (the HMM can be constructed heuristically by an expert).

o Closed form, thoroughly developed, efficient algorithms for state estimation (Viterbi) and model identification (Baum-Welch). 


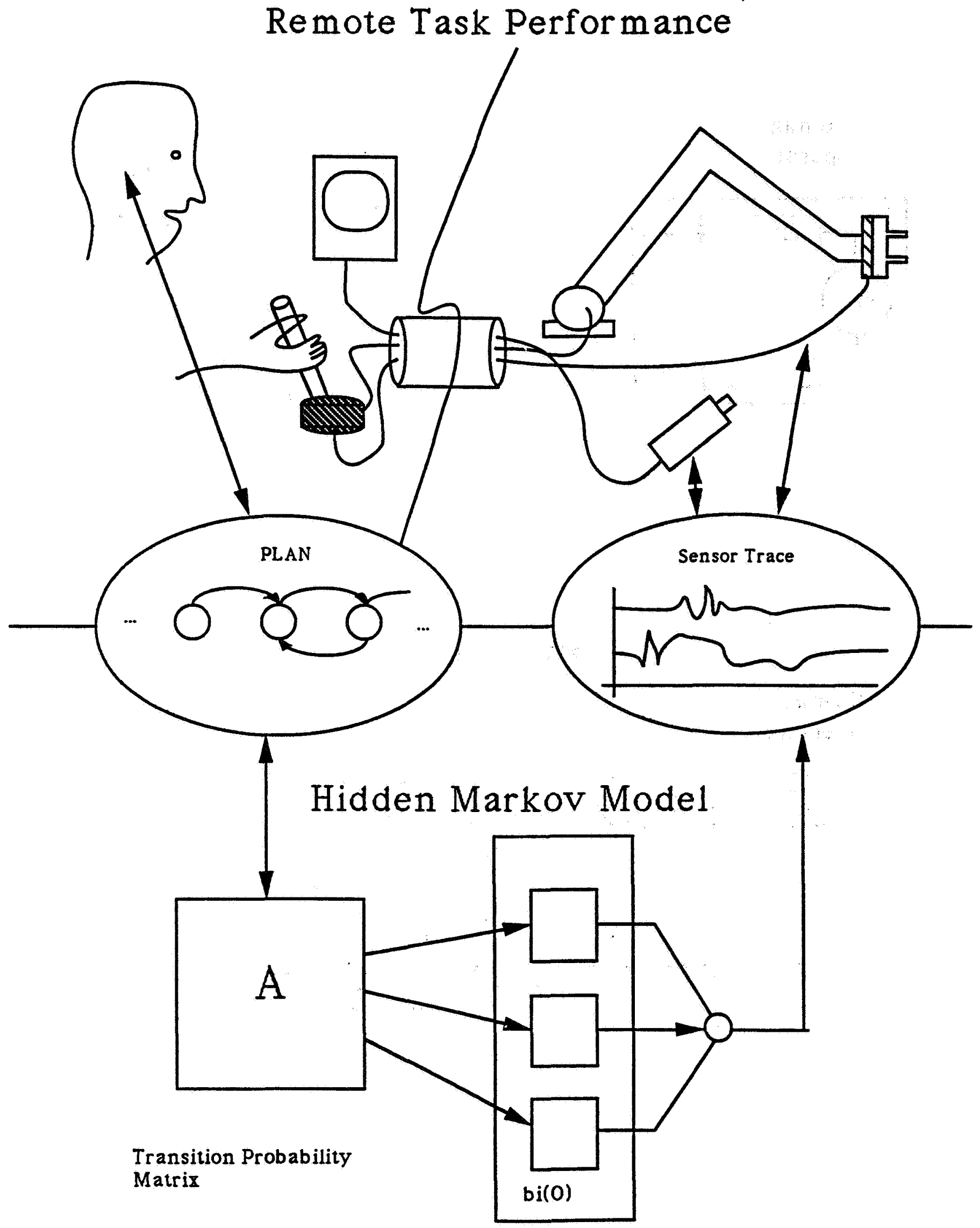

Observation Densities 


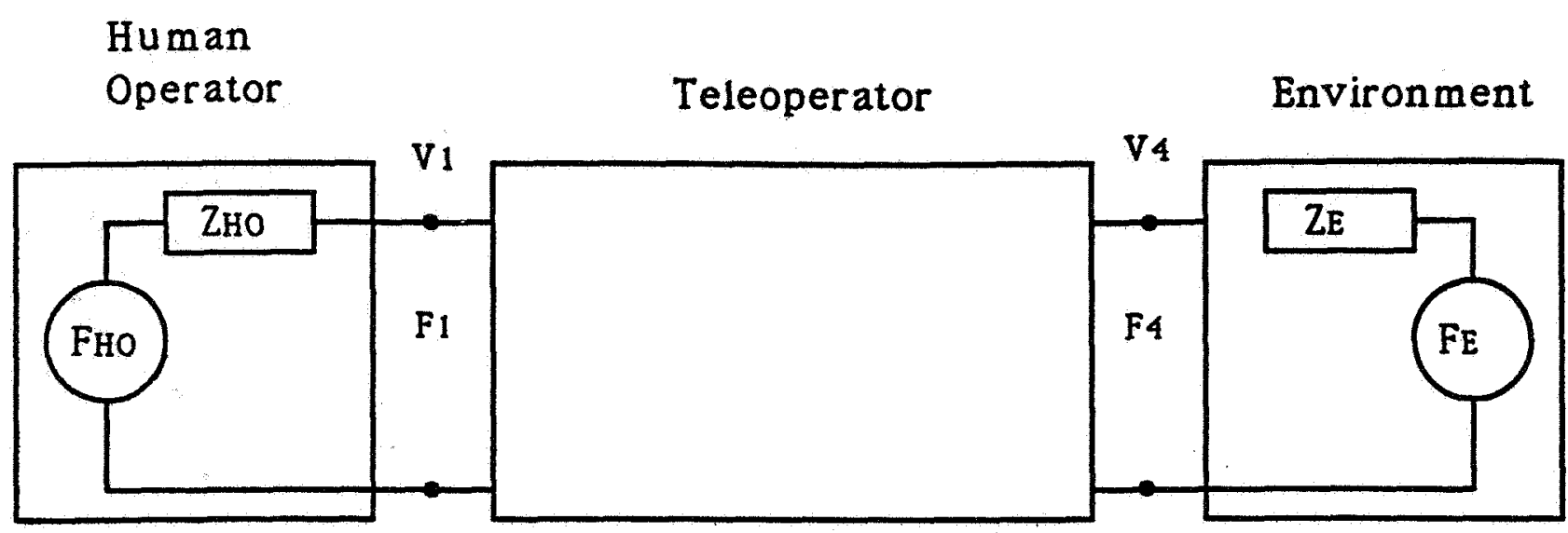

Scaled Teleoperator

Hu man

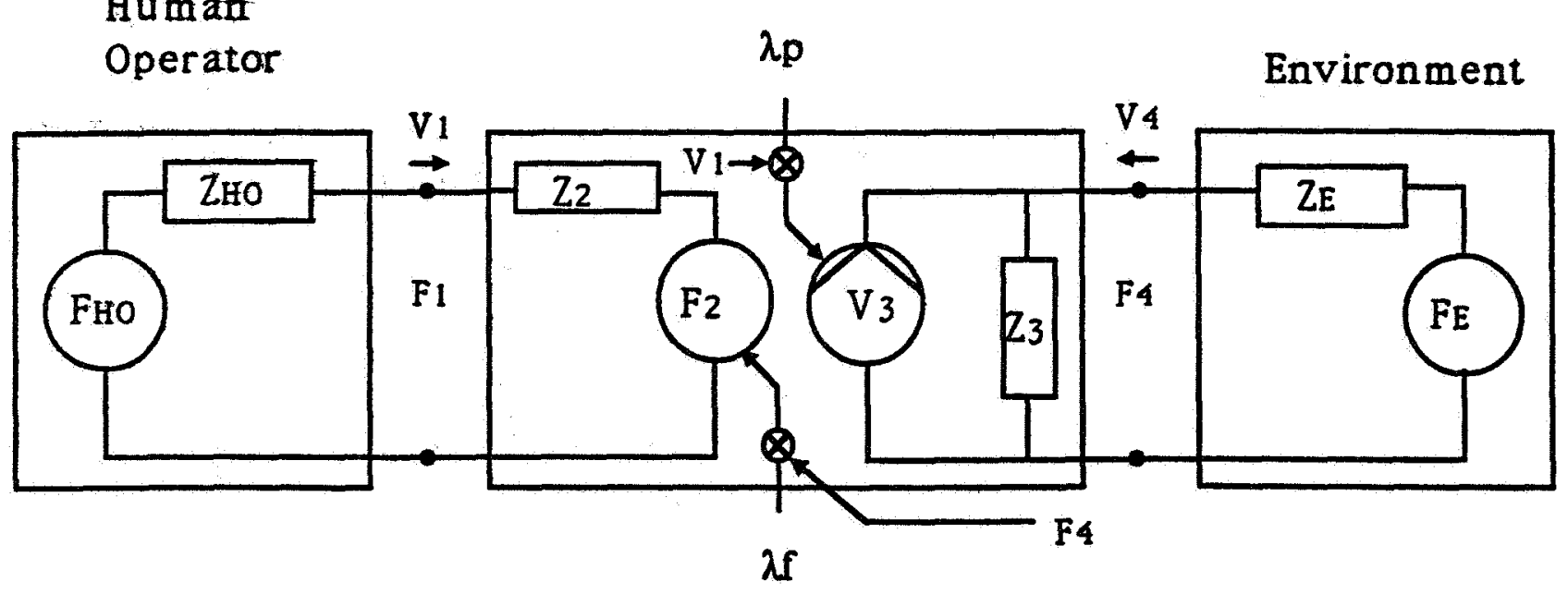




\title{
TELEOPERATING THE SPACE STATIONS
}

\author{
Marvin L. Minsky \\ Massachusetts Institute of Technology \\ Cambridge, Massachusetts
}

(Talk not presented.) 


\section{SOME CRITERIA FOR TELEOPERATORS AND VIRTUAL ENVIRONMENTS FROM EXPERIENCES WITH VEHICLE/OPERATOR SIMULATION}

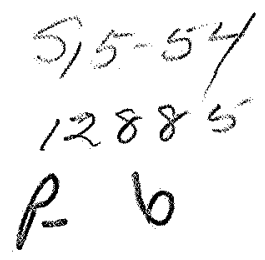

\author{
Henry R. Jex \\ Systems Technology, Inc. \\ Hawthorne, California
}

Over 30 years of experience in the simulation, modeling and analysis of man-machine control systems has resulted in a variety of rules-of-thumb and some quantitative criteria for assuring valid simulation of "real-world" situations and likely users. It is hoped that these criteria, which apply directly to teleoperations (i.e., no communication delays; operator-in-the-loop; no "smart-robot" delegation), will be useful for designing better telerobots and in simulating telerobotic controls and virtual environments.

First, some definitions are given: "Valid simulation" is defined as that producing the same operator behavior, performance and mental workload as the real-world case. "Delay artifact" is the (measured) sum of all throughput delays from: sensing, computing, pipeline and display generation. "Kinetosis" is the vertigo and malaise from conflicts among visual, vestibular and proprioceptive motion cues. "Mental workload" is the operator's evaluation of his attentional-load margin while performing adequately in a specified task context.

A review is given of a wide range of simulations in which operator steering control of a vehicle is involved and the: dominant-cues, closed-loop bandwidth, measured operator effective time-delay, and ratio of bandwidth-to-inverse delay, are summarized. For well designed and low mental workload conditions, this ratio is well under 1.0 , as control theory would dictate. However, for important human tasks like fast locomotion, the ratio approaches 1.0 (limited by the inverse delay limit of about $8 \mathrm{rad} / \mathrm{sec}$ ), and the mental workload becomes critical. These imply requirements that the eyeball stabilization and pursuit subsystems have higher bandwidth. More importantly, it dictates that any simulation delay artifact must be less than about $1 / 4$ of the effective operator delay in a given task, i.e., less than about .04 seconds delay artifact for locomotion simulation.

A correlation of kinetosis (simulator-sickness) with dynamic scene field-of-view (FOV) is shown. It has been found that, to avoid kinetosis, the FOV should span less lateral view than a curve of about $1.10 \mathrm{~Gy}$ would cover at real-world speeds (typically, less than 60 degrees). Other requirements include: framing of scene "streamers" and avoidance of perceivable image jitter or "strobing."

The visual resolution of a virtual environment should allow reading a newspaper subheadlines at arms length. High Definition Television (HDTV) would pass this test.

The use of moving base simulators to improve the validity of locomotion teleoperations is discussed, and data are shown to support the following recommendations. Linear motion bases are not needed if the real-world task entails less than about 1.1 Gy of transverse acceleration. If the rms Gy is greater, it is more important to maintain correct motion-cue phasing ( $<30$ degree disparity) than 
amplitude, which can be cut to about 1/5 the actual levels. Rotary motion rates are sensed and used as "rotary motion dampers" when the real-world task is regulation against inertial motion disturbances (as in running), but are ignored when the task involves pursuit of a maneuvering target. Details are discussed in the presentation.

Some rules-of-thumb for good "feel-system" simulation, such as for control manipulanda are given. Their vernacular version dictates that "good" simulation of a:

- zero inertia and force-free control should "feel like a stick of balsa wood" (negligible friction, jerk or jitter)

- hard stop should "feel like a brick wall" (no creep or sponginess)

- coulomb friction should "feel like sliding a refrigerator magnet" (remain in place; no creep, bounce or jitter)

- centering detent should "yield an audible "klunk" when traversed" (no lag or sponginess)

Try these on your favorite manipulandum!

Finally, simulation tests of teleoperators and virtual environments should include three types of measures: system performance, operator (or robot) "behavior" (e.g., describing functions, etc.), and mental workload evaluations. The criteria for adequate mental workload measures are that they be:

1. Relevant - to mental workload, per se

2. Sensitive - monotonic correlation with workload

- insensitive to irrelevant variables

- high test power (covariance/residual).

3. Concordant - ubiquitous trends in target population

4. Reliable - proven test: retest repeatability

- proven norms and statistics

5. Convenient - easy to administer and learn

- portable

- low cost for adequate level of reliable result

Properly designed subjective ratings usually fare better than such measures as evoked response potentials! Examples of the foregoing approach are given, in optimizing motion washouts.

If your teleoperator or virtual environment simulation can pass these rule-of-thumb tests, then you can be fairly sure that it will provide results which can stand the final test of real-world validation, form a proper data base for scientific modeling and engineering design, and be liked by its users. 
CONTROL BW AND H.Q. DELAYS ARE RELATED

\begin{tabular}{|c|c|c|c|c|c|c|}
\hline \multirow{2}{*}{$\begin{array}{l}\text { SIMULATION } \\
\text { (Example) }\end{array}$} & \multicolumn{3}{|c|}{ DOMINANT CUES } & \multirow{2}{*}{$\begin{array}{l}\text { CLOSED-LOOP } \\
\text { BANDWDTH } \\
\text { BW (r/s) }\end{array}$} & \multirow{2}{*}{$\begin{array}{l}\text { TYPICAL } \\
\text { OPERATOR DELYY } \\
\text { t(sec) }\end{array}$} & \multirow{2}{*}{$\frac{B W}{T^{-1}}$} \\
\hline & VISUAL & MOTION & FEEL & & & \\
\hline $\begin{array}{l}\text { Ship Conning } \\
\text { (SES-2000) }\end{array}$ & $\checkmark$ & Disturb & - & $.01-.10$ & $2-5$ & 20 \\
\hline $\begin{array}{l}\text { Blimp mooring } \\
(S-500)\end{array}$ & $\checkmark$ & - & - & 3 & 5 & .15 \\
\hline $\begin{array}{l}\text { Manual Booster Control } \\
\text { (Salum V) }\end{array}$ & $\checkmark$ & Disturb & Disturb & 1.5 & 3 & .45 \\
\hline $\begin{array}{l}\text { Transport Aircraft } \\
\text { (Boeing 707) }\end{array}$ & s & Some & $\checkmark$ & 20 & 25 & 5 \\
\hline $\begin{array}{l}\text { Fighter Aircraft } \\
(F-16)\end{array}$ & $d$ & $\checkmark$ & $\checkmark$ & $4.0+$ & $.15-22$ & .7 \\
\hline $\begin{array}{l}\text { Automobiles } \\
\text { (Honda) }\end{array}$ & $\checkmark$ & $\checkmark$ & $\checkmark$ & $4.0+$ & 25 & 8 \\
\hline $\begin{array}{l}\text { Bicycie (slow) } \\
\text { (Recumbent Bike) }\end{array}$ & $\checkmark$ & $\checkmark$ & $\checkmark$ & 6.0 & .15 & 9 \\
\hline $\begin{array}{l}\text { Cursor Aiming } \\
\text { (Critical Instability Task) }\end{array}$ & $\checkmark$ & - & - & $6.0-6.0$ (limil) & $.12-20$ & 1.0 \\
\hline Eye - Pursuit Loop & $\sqrt{ }$ & - & - & 10 & .06 & .6 \\
\hline Eye - Vestibulo-ocular & $?$ & $\sqrt{ }$ & $?$ & $30+$ & .02 & .6 \\
\hline
\end{tabular}

\section{IMPLICATIONS - VISUAL DELAYS}

- Optimized control tasks have $\mathrm{BW} / \mathrm{T}^{-1} \ll 1.0$

- Human will adapt $\tau_{\text {eff }}$ to $\approx 1 / 5 \mathrm{BW}$, down to $\tau \approx .20 \mathrm{sec}$

- For high $B W$ tasks: $B W / \tau^{-1} \approx 1.0$ and $\tau_{\min }>.16 \mathrm{sec}$

- Control theory shows that any simulation delay artifacts must be «20 sec to provide "valid" simulation

\section{Criterion 1}

- $\quad \therefore$ Valid" simulation requires delay artifacts be $\ll .20$ sec:

$<.02 \mathrm{sec}(6 \mathrm{HH})=$ Excellent; $<.04 \mathrm{sec}=$ Good; $>.10 \mathrm{sec}=$ Invalid 


\section{FIELD OF VIEW VS. KINETOSIS}

\section{Fixed-Base Vertigo vs. Field of View}

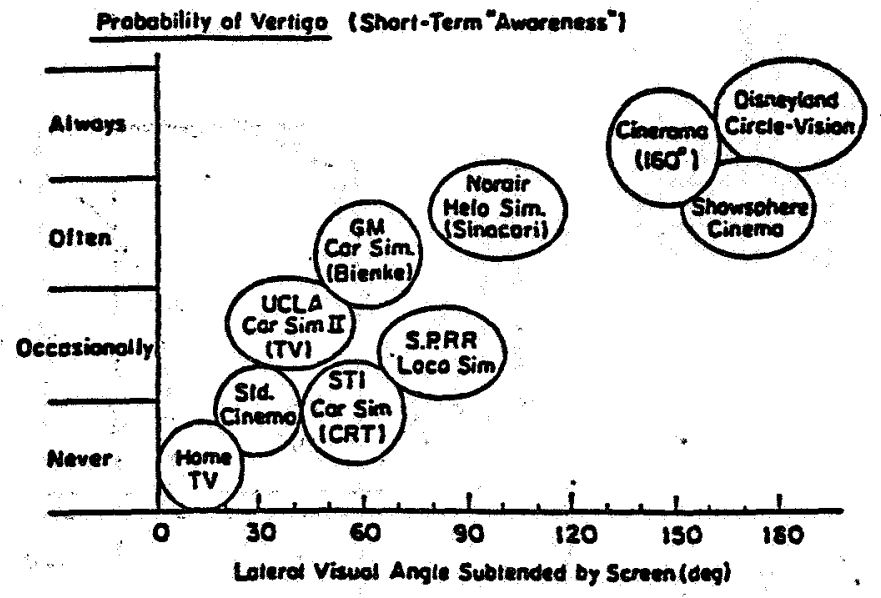

Implications; For wide FOV use "impoverished scene" Criterion 2: Keep FOV to cover less lateral view than a curve of $\pm .10 \mathrm{G}_{y}$ would
cover at real-world speeds

Other factors:

- More parafoveal details $\rightarrow$ worse conflict

- Proper framing of parafoveal streamers is important

\section{VISUAL UPDATE AND RESOLUTION OF DISPLAYS}

Criterion

- For "inner loop" control of path rate and curvature, "streamers" from scene microtexture should not jerk or strobe backward. ( $20-60$ frames/sec update)

Criterion

- For "outer loop" guidance and navigation must be able to perceive and understand cues at $\approx 3-5 \mathrm{sec}$ ahead of vehicle Implies $\approx 2$ min-arc of resolution for signs, potholes, targets (e.g., HDTV)

Criterion

- For Virtual Environment should be able to read a newspaper at arm's length. (Subheadlines $=$ OK) (e.g., HDTV) 

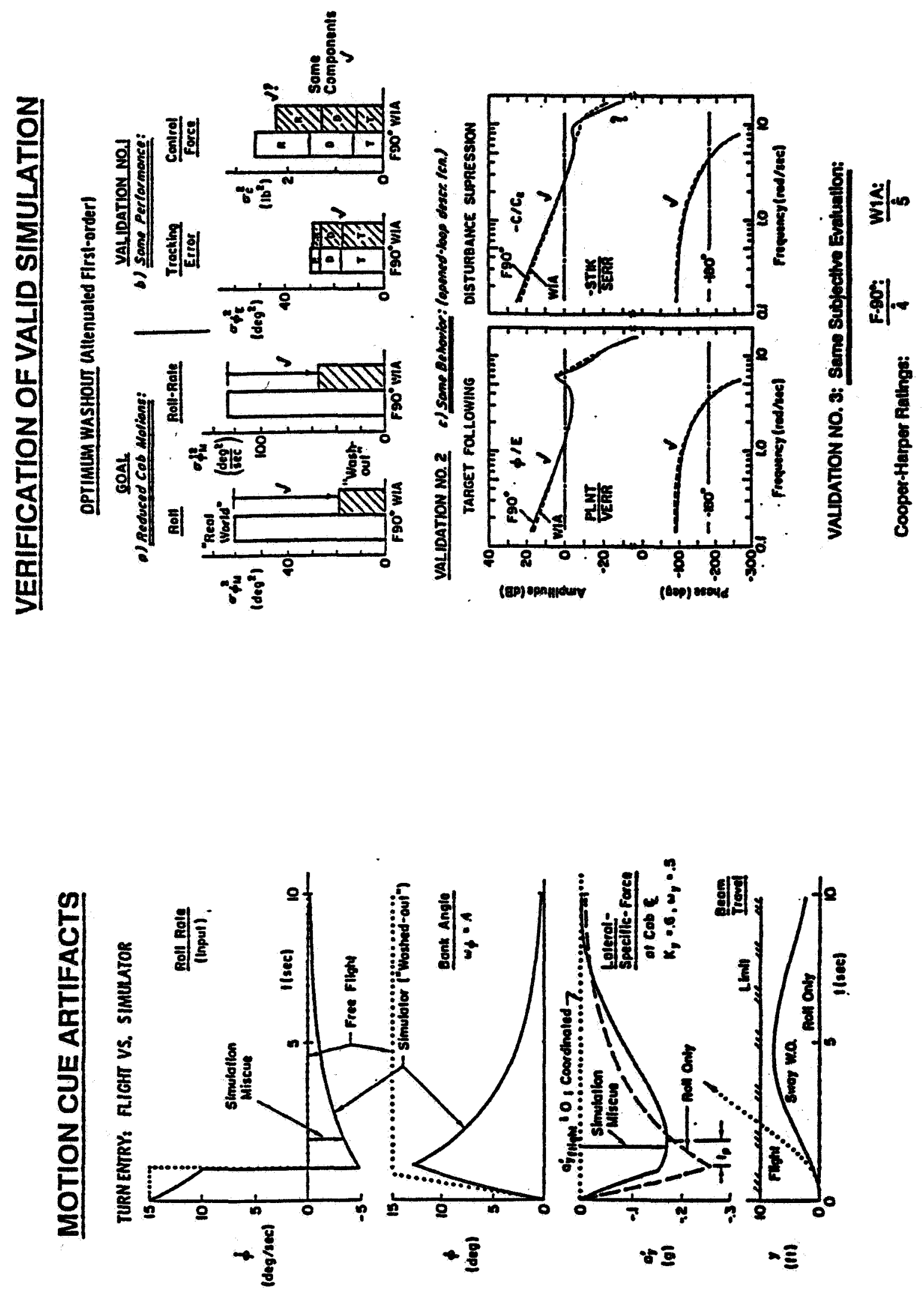

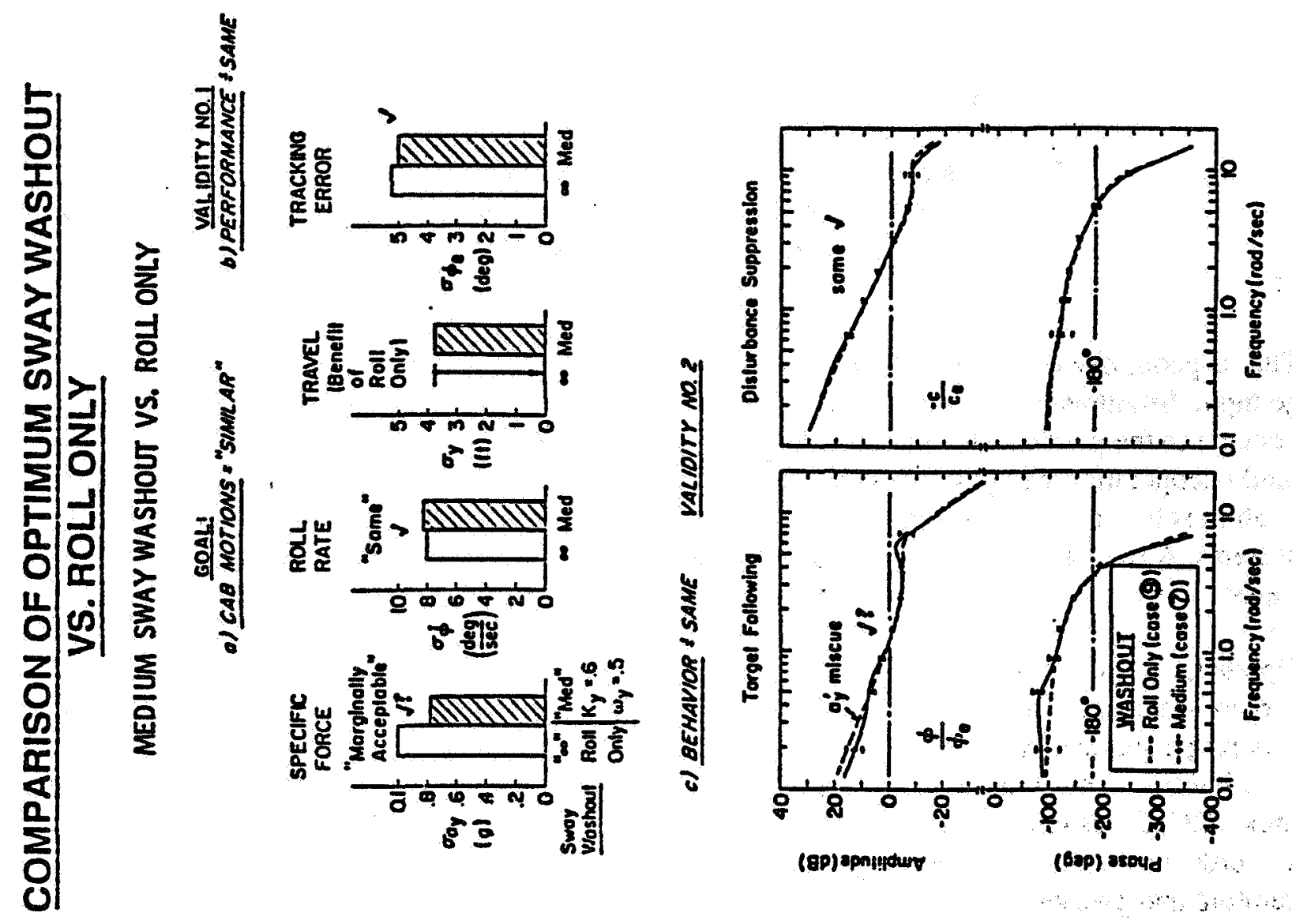

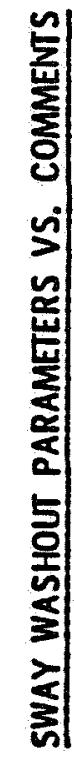
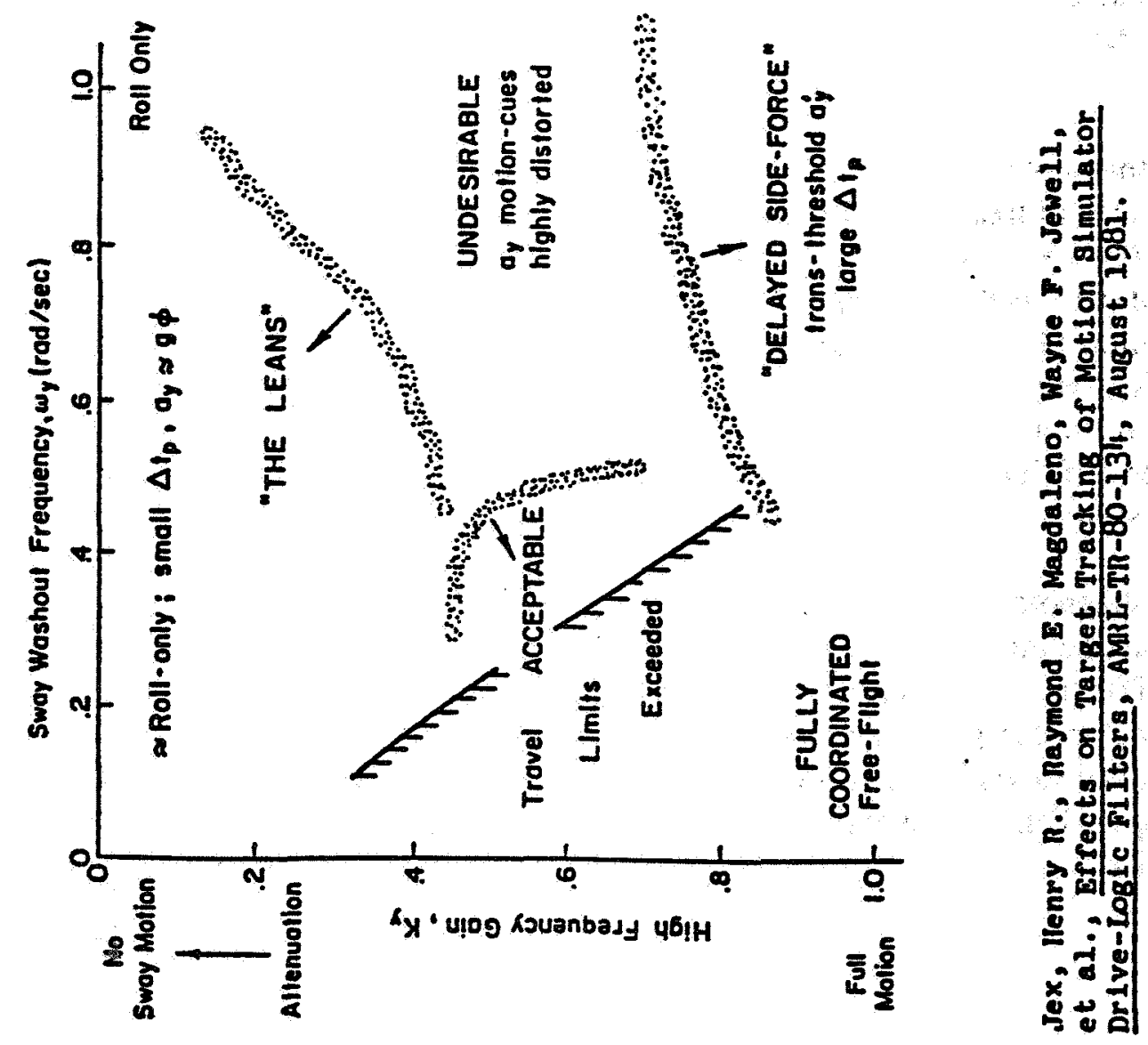


\title{
MAN-MACHINE INTERFACES IN HEALTH CARE
}

\author{
Steve Charles, M.D. and Roy E. Williams \\ Center for Engineering Applications \\ Memphis, Tennessee
}

The surgeon, like the pilot, is confronted with an ever increasing volume of voice, data and image input. Simultaneously, the surgeon must control a rapidly growing number of devices to deliver care to the patient. The broad disciplines of man-machine interface design, systems integration and teleoperation will play a role in the operating room of the future. The purpose of this communication is to report the incorporation of these design concepts into new surgical and laser delivery systems. A review of each general problem area and the systems under development to solve the problems follows.

Ophthalmology is a machine-dependent specialty embracing the patient-machine interface and the surgeon-machine interface. As many as fifteen independent systems may be used in the operating room during a surgical procedure. Each system is currently manually controlled creating an intensive operator interface environment. The surgical operating system known as the Ocular Connection Machine (OCM) has reduced the number of stopcocks, foot pedals, adapters, displays, controls and power cords by several orders of magnitude. Physiological analog parameters, including intraocular temperature and pressure, previously monitored with human interaction are now controlled by servo systems, while surgical analog parameters, i.e., cutting and thermal, are controlled by dual proportional positioning of the surgeon's foot. Multiple displays have been replaced by one thus reducing viewing time and errors. By reducing the amount of man-machine interactions, the system markedly reduces set-up time and clean-up time, as well as improving reliability, functionality and patient safety.

Laser treatment of the retina is an intensive man-machine task. The ophthalmologist must determine the area of treatment, aim the beam and fire while the patient is awake. The Laser Imaging Work Station (LIWS) currently under development alleviates much of the ophthalmologist-machine interaction and reduces the discomfort of the patient-machine interaction. The LIWS is an integrated system containing a high-speed digital correlation retinal tracker, an automated laser delivery system which controls laser power, wavelength, spot size, duration and location, and an expert system with algorithms to avoid the macula, optic nerve, vessels and previous photocoagulated areas. Surgeon interface occurs off-line whereby a patient treatment template is determined and down-loaded to the laser delivery system.

Real-time diagnostic imaging of a patient's eye is currently resolution limited, two-dimensional and user interaction intensive. A system is currently under development which provides quasi-real time stereo (3D) ultrasound and improved lateral resolution. Depth of the viewing volume within the eye (focal plane) is identical to current focus mechanisms used by ophthalmologists whereby a handheld lens is moved forward and backward yielding different viewing planes (indirect ophthalmoscopy). This hand-eye coordination and resulting spatial information will be natural for ophthalmologists. 
Utilization of advanced robotic systems in a teleoperation environment will enable microsurgeons to improve present operations and allow those surgeons not as dexterous to perform operations not manageable with present systems. For example, epiretinal membranes too thin to remove without risk of macular holes would be a realizable target for the ophthalmic microsurgeon. By applying 6 degrees-of- freedom in a coarse-fine strategy with translational resolutions of 10 to 20 microns, these techniques and others are made possible. The surgeon controls the bilateral robotic system through the surgeon-machine interface (SMI) which provides stiffness feedback by sensing force and position at the operating site and backdriving actuators within the SMI. Further sensing of optical, electrical and mechanical impedance at the operation site is fed back to the surgeon via the foot pedal interface, offering another realm of sensory feedback. 


\section{TELEROBOTICS IN REHABILITATION: BARRIERS TO A VIRTUAL} EXISTENCE

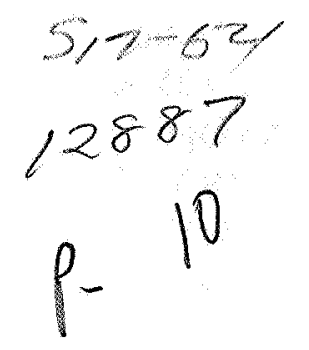

\author{
Larry Leifer, Machiel Van Der Loos, and Stefan Michalowski \\ Stanford Center for Design Research \\ Stanford, California
}

\section{The Need for Telerobotics in Rehabilitation}

There are over 67,500 quadriplegics in the United States today, with an estimated 2,400 to 4,000 new injuries occurring each year (Stover et al., 1986). These injuries occur most frequently among young males between the ages of 16 and 30. Due to advances in medical treatment (antibiotics and skin care), these individuals now have a relatively normal life expectancy. They are alive, but dramatically cut off from most aspects of a normal existence. They live, virtually, through the actions of others. Perhaps, life would be more fulfilling if they were also offered the opportunity to directly control their environment through telerobotic tools for independent living. Virtual telerobotic environments for the rest of us could bring reality to these individuals.

It is estimated that caring for a quadriplegic, including standard medical treatment, equipment maintenance and attendant care, costs about $\$ 47,000$ (1980 dollars) per year. This translates to approximately 1.9 million dollars over forty years (Kalsbeek et al., 1980). The net direct cost to the Department of Veterans Affairs will be approximately $\$ 5$ billion for its current population of service connected quadriplegics. On the promising side, Young et al (1982) estimated that every dollar spent for rehabilitation research and development returns $\$ 11$ in cost benefits to society. In the case of quadriplegia, the cost of attendant care (nominally $\$ 25 / \mathrm{hr}$ to the insurer and increasing) can be reduced by providing personal telerobots (nominally $\$ 5 / \mathrm{hr}$ and decreasing). Hammel et al. (1989) demonstrated that telerobots can satisfy the vocational manipulation needs of personal computer users for periods of over four hours at a time without attendant intervention. Employment makes it possible to recover some or all of the indirect cost of severe disability.

\section{Barriers to Telerobotics Technology in Rehabilitation and Health Care}

If the direct cost of severe disability is so high, and telerobotics technology is available to help reduce costs, then what have been the barriers to its widespread acceptance and deployment? Clinical experience with telerobots suggests that there are several key barriers:

Social Barriers: As a society, we place little emphasis on restoration of function for persons with disability, we prefer to "take care of them". Because the economics of cost and benefit are not coupled, we fail to see the opportunity. However, even if we began deployment today, our society has educated too few persons to support the advanced assistive devices (i.e., we have enough researchers to create independent living tools, but too few development and service persons to support clinical and domestic usage). These are the dominant factors impeding wider adoption of advanced technical aids for persons with disability. 
Institutional Barriers: Government programs are scattered and disjoint. There is no systematic vision and considerable inter-agency protectionism. Perhaps most significantly, the "conflict of interest" witch hunt in government makes it virtually impossible to transfer laboratory results to the commercial sector in a timely and cost-effective manner. For its part, the commercial sector does not take the investment risk to develop these devices because it is not clear that third party reimbursement will be forthcoming.

Technical Barriers: Here, at least, the science and engineering communities do have some control of the issues, these include:

The human-machine-interface is the dominant technical barrier to widespread use of telerobots in rehabilitation. Text, voice, graphic and kinematic command-control interfaces are very cumbersome for robot motion specification, planning and supervision. This forces the user to be overly dependent on pre-programmed motions and the technicians who create them. We must work towards "instructable" telerobots.

The machine-environment-interface is the second most deficient aspect of telerobotics in rehabilitation. The absence of sensor driven grasp and object approachavoidance reflexes forces the user to directly control end-effector motion under difficult observational circumstances and without "natural kinesthetic cues". We must develop robust sensate end-effectors and the "reflexes" to make them useful. Force (impedance) control will be a requirement for advanced user support tasks.

Mobility, or the lack of it, defines the telerobot's work space and, in part, its ultimate utility. One can not reasonably expect general cost-effectiveness when people must be available to bring work to the telerobot. Raw mobility is, however, not enough. Remote presence makes much greater demands on the user-interface and telerobotic sensory capability. A more "intelligent" robot may, in fact, be the greatest challenge yet to the user-interface designer.

Fault-intolerance is an overriding shortcoming of almost all robots. As programmable electro-mechanical systems, they are inherently subject to a very wide variety of fault modes. Not even the digital controller in current machines take advantage of computer fault-tolerance architectures (which themselves make no provision for sensor and actuator failure modes). Widespread personal use of telerobots will require fundamental progress in design for fault-tolerance (we must get well beyond just being careful). 


\section{Telerobotics in Rehabilitation: Once Around the World}

A small number of underfinanced telerobotics teams around the world are attempting to overcome these barriers. The most recent compilation of papers are in the Proceedings of the "First International Workshop on Robotic Applications in Medicine and Health Care" (1988). The next edition will grow out of the International Conference on Rehabilitation Robotics (sponsored by the A. I. duPont Institute, June, 1990). The following synopses highlight some of the ongoing R\&D (see Table-4 for a technical comparison of the telerobots used.

The Johns Hopkins University Applied Physics Laboratory (JHU/APL) project (Schneider et al., 1981) concentrated on the implementation of a workbench-mounted robot intended to perform activities of daily living (ADL) tasks. The design (initially 4 degrees-of-freedom then extended to 5) was derived from prosthetic arm technology. Control is accomplished by a head-motion (chin) controlled joystick for joint specific motion. Pre-programmed motions are invoked by menu-selection and input command via a sip-and-puff switch.

The Tufts-New England Medical Center robotics project (Gilbert and Foulds, 1987; now at the A I duPont Institute) concentrated on the design of a universal robot programming language, CALVIN, to provide a common interface to the many different manipulators available. Using CALVIN, they set up a variety of small, low cost, robot work cells (typically 4 and 5 degree-offreedom manipulators) in clinical rehabilitation settings for disabled children. The clinical objective is to foster intellectual development of the child.

The Boeing Company developed a voice-controlled workstation using the Universal Machine Intelligence RTX ( 5 degree of freedom) manipulator ( $\mathrm{Fu}, 1986$ ). The distinctive feature of this project is that it's user interface is a voice query system for large data bases developed by Boeing. Prab Command, Inc., began marketing the system in 1988 for vocational applications. There is very little telerobotic function beyond pre-programmed diskette loading. The internal project at Boeing has ended and Prab sold the technology to the Zenith-Heath.

From 1980 through 1988, the Veterans Administration sponsored a collaborative effort with Stanford University (Leifer, 1982; Hammel et al., 1989) to test the hypothesis that industrial manipulators could cost effectively serve the needs of severely impaired spinal cord injury patients. The project demonstrated that, through voiced commands, the utility and reliability of a high performance manipulator (PUMA-260) and control language (VAL-2) can yield attractive cost-to-benefit performance ratios when the telerobot is able to operate for four hours without attendant intervention. Sensate end-effector, mobility, 3D point designation and natural language studies laid the foundation for further R\&D. A desktop vocational assistant robot (DeVAR) version of the system is available commercially.

Outside the United States, the Canadian Neil Squire Foundation has developed a low-cost manipulator designed for desktop applications in rehabilitation (Cameron, 1986). The system is being sold by the foundation. At the Institute for Rehabilitation Research in the Netherlands, Hok Kwee (previously with the French Spartacus project (Kwee, 1983) and his colleagues have developed a wheelchair mounted, joystick-controlled, manipulator (MANUS, Kwee, 1987). They are 
particularly interested in collocation of the robot and user at all times. The manipulator is expected to function as an arm, not really a telerobot.

While human attendant care is the norm today, and telerobotics technology promises to release some attendant time cost effectively, there is a third alternative. Capuchin monkeys have been trained to provide attendant services from feeding to appliance operation (internal VA program review, 1988). Monkey training is expensive, labile, and done by methods objectionable to the animal rights community. Program retention (to a maximum of 12 short tasks) and willfulness remain significant obstacles to the monkey assistant concept. The approach is particularly limited by the fact that monkeys can only work one-on-one with their user. The distractions of institutional and vocational settings render the monkey ineffective.

Table 1 presents an extended comparison of the strengths, capabilities and limitations of five approaches to augmenting the independence of severely impaired persons. Supported by a growing body of experimental evidence, this comparison strongly suggests that telerobots can become costeffective personal and vocational assistants. Table 2 lists many of the feeding, personal hygiene, vocational, and recreational tasks that have been demonstrated over the past 8 years by four generations of the Stanford-VA Rehabilitation R\&D Center's Telerobotic systems. Table 3 identifies the technical functions required to perform these tasks.

\section{A Partial View of the Future}

The operator interface will remain the dominant problem. It is so difficult that most systems designers prefer to ignore it and focus on "tangible" technical specifications. It is likely to use multiple-channels in the sense of incorporating a "natural robot instructional language" and bi-directional pictographic dialog between the operator and the system. Increasingly "autonomous" telerobots will actually increase the burden on this interface.

Sensor driven motion planning and autonomous grasp/avoidance reflexes will become commonplace. The rate of introduction of such features will, however, be much slower than expected. In part, this is due to the fact that system architectures will continue to be "fault-intolerant" such that the introduction of both sensors and programmed reflexes will bring new reliability problems with them.

Force (impedance) control of telerobots will continue to evolve slowly even thought this capability is a fundamental requirement for any robot that must work intimately with humans. Physical therapy by robots is both needed and impossible without force control. These lines of technical evolution will themselves depend on getting more applications feedback from telerobots in the clinic, home and office. In combination, we see a rather daunting challenge.

\section{Acknowledgment}

The following individuals have made important contributions to the reasoning in this paper: Joy Hammel, David Lees, Dean Chan, Inder Perkash, Lin Liang, David J. Cannon, Charlie Wampler, Chuck Buckley, John Jameson, Doug Schwandt, and Jim Anderson. 


\section{References}

Cameron, W., Manipulative Appliance Development in Canada in Interactive Robotic Aids - one option for independent living, World Rehabilitation Fund, New York, 1986

Fu, C., An Independent Vocational Workstation for a Quadriplegic, Proceedings of the 9th Annual RESNA Conference, Minneapolis, MN, 1986

Gilbert, M., and Foulds, R., Robotics at Tufts-New England Medical Center: a progress report, Proceedings of the 10th Annual RESNA Conference, San Jose, CA, 1987

Hammel, J., Hall, K., Lees, D., Leifer, L., Van der Loos, M., Perkash, I, and Crigler, R., Clinical evaluation of a Desktop Robotic Assistant, Journal of Rehabilitation Research and Development, Vol. 26, No.3, 1989

Kalsbeek, W., McLaurin, R., Harris-III, B., and Miller, D., The national head and spinal cord injury survey: Major Findings, Journal of Neurosurgery, Vol. 53, 1980, pp. S19-S31

Kwee, H. H., et al., Development of the MANUS Wheelchair-Borne Manipulator: progress report, Proceedings of the 10th Annual RESNA Conference, San Jose, CA, 1987

Kwee, H. H., First Experimentation with the Spartacus Telethesis in a Clinical Environment, Paraplegia, vol. 21, 1983

Leifer, L. J., Restoration of Motor Function: a robotics approach, in Uses of Computers in Aiding the Disabled, Amsterdam: North-Holland Co., 1982

Schneider, W., Schmeisser, G., and Seamone, W., A Computer-Aided Robotic Arm-worktable System for the High-Level Quadriplegic, IEEE Computer, pp. 41-47, January, 1981

Stover, S. L., Fine, P. R., et al: Spinal Cord Injury: The Facts and Figures, University of Alabama, 1986

Van der Loos, H. F. M, Michalowski, S. J., Hammel, J., Leifer, L. J., and Stassen, H. G., Assessing the impact of robotics devices for the severely physically disabled, Proceedings of the First International Workshop on Robotic Applications in Medical and Health Care, Ottawa, Canada, 1988, pp. 1-6

Young, J., Burns, P., Bowen, A., and McCutchen, R., Spinal Cord Injury Statistics, Phoenix, Arizona, Good Samaritan Medical Center, 1982

Proceedings of the First International Workshop on Robotic Applications in Medical and Heaith Care, Editorial Office Rm. 301, Electrical Engineering, National Research Council, Ottawa, Canada, K1A 0R8, 1988 
Table 1a In-depth Review of the Capabilities, Strengths and Weaknesses of Five Basic Approaches to the Restoration of Manipulative Function for People with Severe Physical Limitations (none = -, limited $=*$, usable $=* *$, good $=* * *$, excellent $=* * * *$ ).

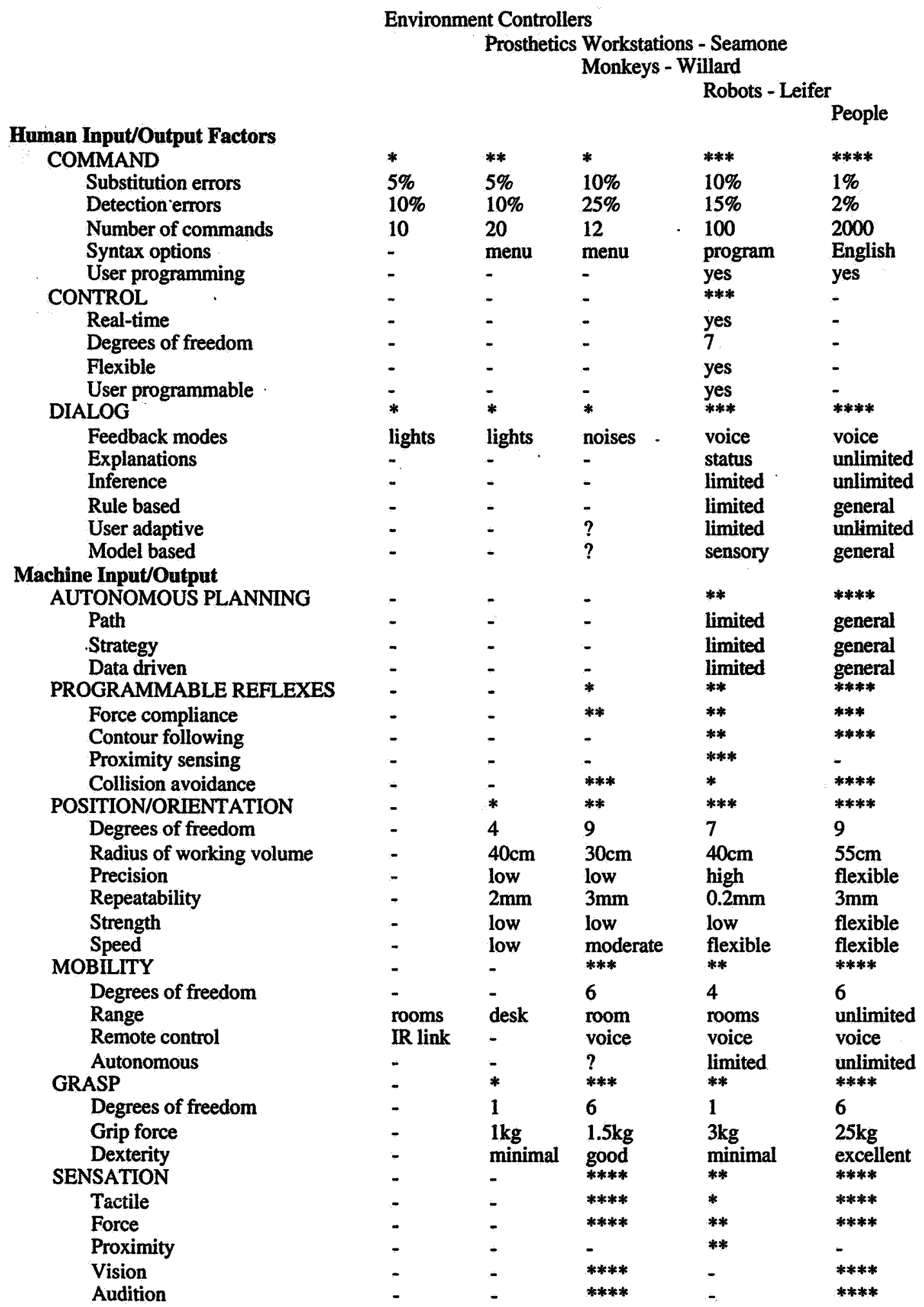


Table 1b

Environment Controllers

Prosthetics Workstations - Seamone Monkeys - Willard

\section{Assessment}

PERFORMANCE

Task time

Training time

Number of tasks

Commands per task '

Precision

Repeatability

Reliability

Accessibility

SAFETY

Intention errors

Intrusion errors

Contact errors

\section{COST}

Hardware

Software

Training

Maintenance

Cost/hour of use

$\begin{array}{lllll}* * & * * & * * & * * * & * * * \\ \text { seconds } & \text { minutes } & \text { minutes } & \text { minutes } & \text { seconds } \\ 2 \mathrm{hrs} & 30 \mathrm{hrs} & 20 \mathrm{hrs} & 40 \mathrm{hrs} & 4 \mathrm{hrs} \\ 10-20 & 15-30 & 10-20 & 20-200 & 40-400 \\ 1 & 1-4 & 1-4 & 1-20 & 1-2 \\ - & 5 \mathrm{~mm} & 2 \mathrm{~mm} & 0.2 \mathrm{~mm} & 2 \mathrm{~mm} \\ - & 1 \mathrm{~mm} & 3 \mathrm{~mm} & 0.2 \mathrm{~mm} & 3 \mathrm{~mm} \\ \text { high } & \text { good } & \text { fair } & \text { good } & \text { fair } \\ 24 \mathrm{hrs} & 24 \mathrm{hrs} & 12 \mathrm{hrs} & 24 \mathrm{hrs} & 8 \mathrm{hrs} \\ * * * * & * * * & * * & * * & * * * * \\ 5 \% & 10 \% & 5 \% & 10 \% & 4 \% \\ 0 \% & 4 \% & 5 \% & 5 \% & 2 \% \\ 0 \% & 2 \% & 4 \% & 2 \% & 1 \% \\ * * * * & * * & * * & * * & * \\ \text { low } & \text { medium } & \text { medium } & \text { high } & \text { low } \\ \text { low } & \text { low } & \text { high } & \text { high } & \text { low } \\ \text { low } & \text { medium } & \text { high } & \text { medium } & \text { low } \\ \text { low } & \text { medium } & \text { medium } & \text { low } & \text { high } \\ \$ 1 / \mathrm{hr} & \$ 4 / \mathrm{hr} & \$ 10 / \mathrm{hr} & \$ 5 / \mathrm{hr} & \$ 25 / \mathrm{hr}\end{array}$


Table 2 Meal preparation and service, vocational material handling, personal hygiene, and recreational tasks that have been performed for and with disabled individuals across four generations of desktop robotic assistants (DeVARs I, II, III, IV)

\begin{tabular}{|c|c|c|}
\hline $\begin{array}{l}\text { arrange table setting } \\
\text { open and close microwave door } \\
\text { open and close refrigerator door } \\
\text { manipulate containers } \\
\text { set appliance timer } \\
\text { pour liquids and solids } \\
\text { beat eggs } \\
\text { toss salad } \\
\text { soup preparation and service } \\
\text { heat and serve casseroles } \\
\text { serve pudding } \\
\text { serve fruit } \\
\text { prepare and serve spaghetti } \\
\text { prepare and bake a cake } \\
\text { use knives, forks and spoons } \\
\text { retrieve drinks } \\
\text { mix drinks } \\
\text { lock and unlock cabinet doors } \\
\text { light and extinguish candles } \\
\text { light and extinguish cigarette } \\
\text { open and close storage drawers } \\
\text { room lights } \\
\text { window open and close }\end{array}$ & $\begin{array}{l}\text { Vocational material handling } \\
\text { write with pen and/or pencil } \\
\text { retrieve books and manuals } \\
\text { set up books for reading } \\
\text { turn book and report pages } \\
\text { turn on-off computer equipment } \\
\text { type on keyboard } \\
\text { adjust keyboard position } \\
\text { operate private and speaker phones } \\
\text { insert and retrieve diskettes } \\
\text { insert and retrieve audio tapes } \\
\text { operate dictation equipment } \\
\text { manipulate printout } \\
\text { voiced control of generic software } \\
\text { load and operate printer } \\
\text { retrieve and serve medication } \\
\text { circuit boards for inspection } \\
\text { operate electronic control units: } \\
\text { door operation } \\
\text { security system } \\
\text { stereo equipment } \\
\text { Recreation } \\
\text { paint and sketch } \\
\text { arrange flowers } \\
\text { hand out flowers } \\
\text { hand out candy and souvenirs } \\
\text { perform one armed ballet } \\
\text { checkers } \\
\text { monopoly } \\
\text { television } \\
\text { operate video games } \\
\text { pac-man } \\
\text { invaders } \\
\text { play board games: } \\
\text { chess }\end{array}$ & $\begin{array}{l}\text { Personal hygiene } \\
\text { wash and dry face } \\
\text { brush teeth } \\
\text { dispense toothpaste } \\
\text { use electric shaver } \\
\text { retrieve mouthstick } \\
\text { comb and brush hair } \\
\text { blow nose } \\
\text { apply makeup }\end{array}$ \\
\hline
\end{tabular}


Table 3. Several functional capabilities are needed in a Telerobotic Assistant to achieve utility in the performance of independent living tasks, not needed ( ), should have ( $($ ), must have ( $($ )

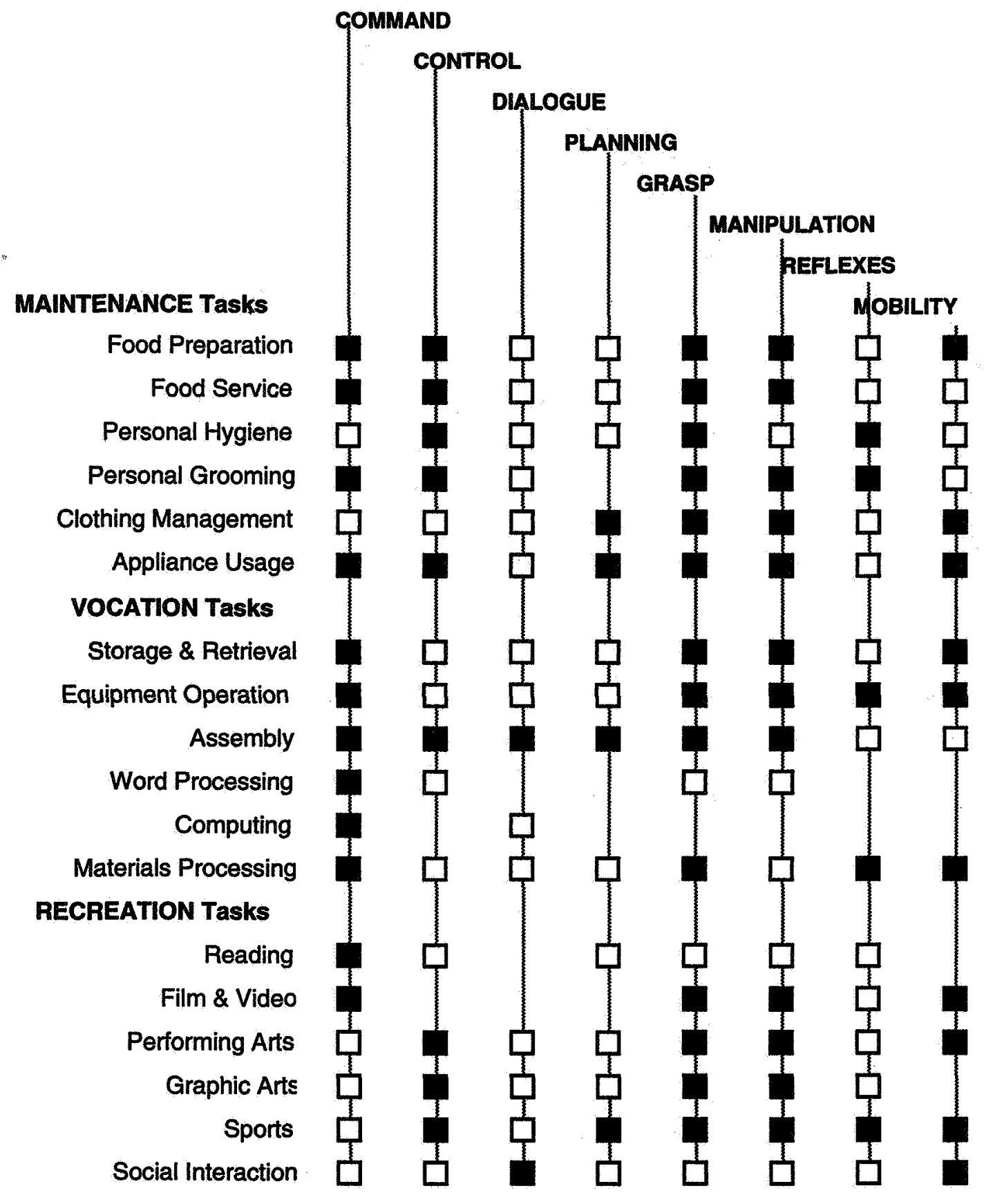




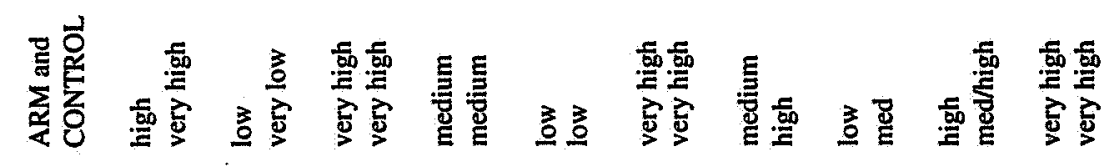

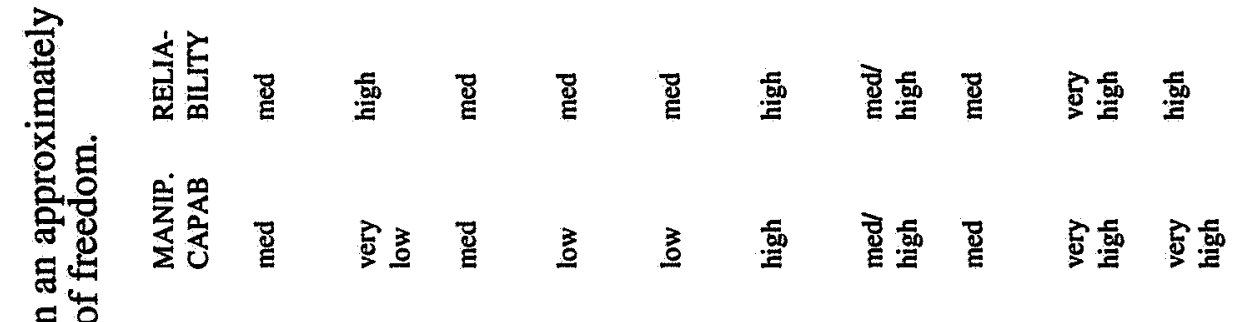

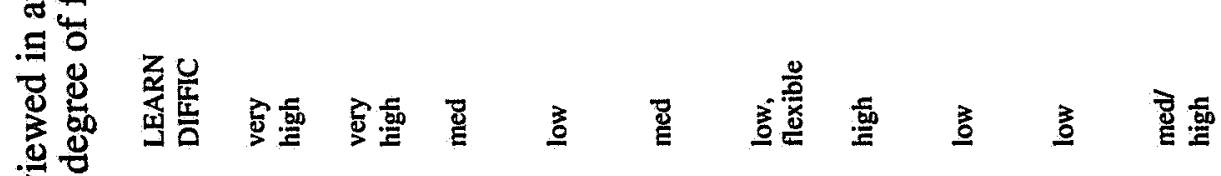

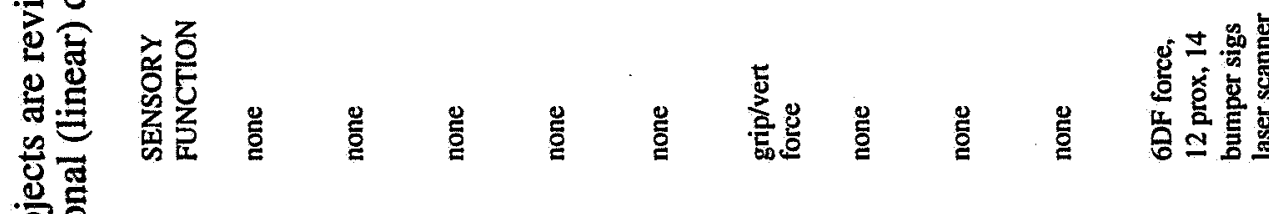

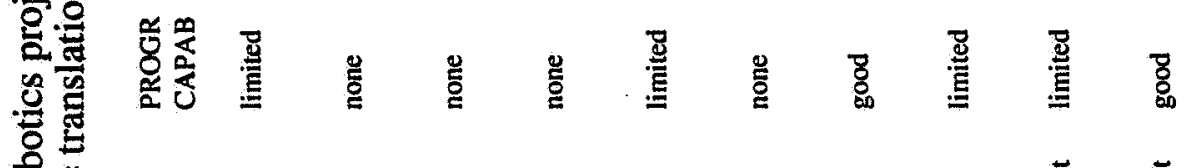

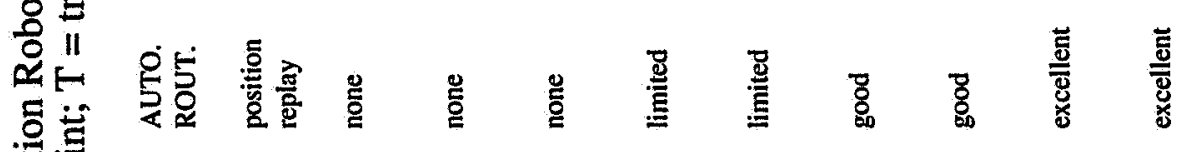

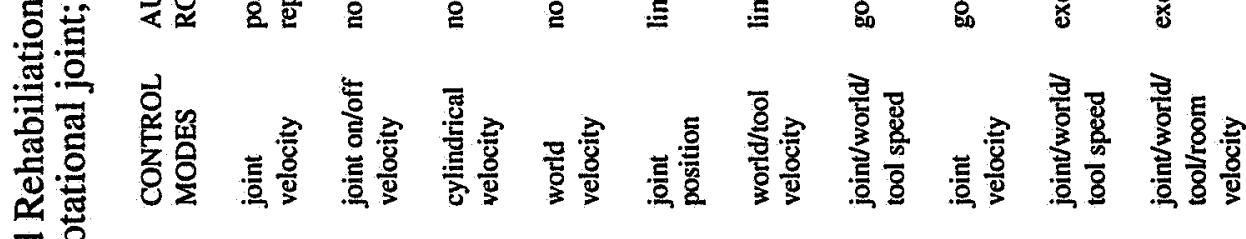

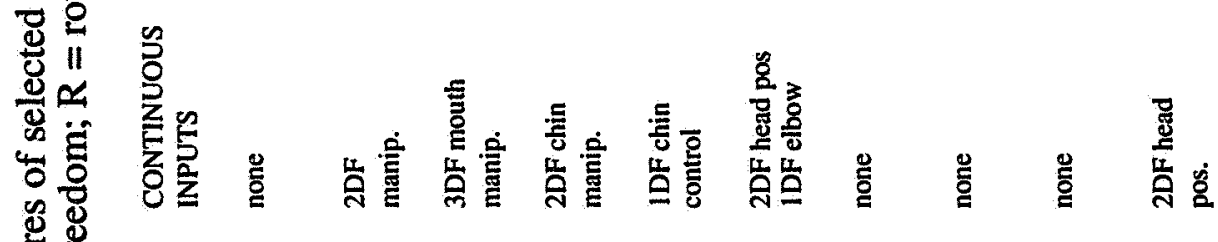

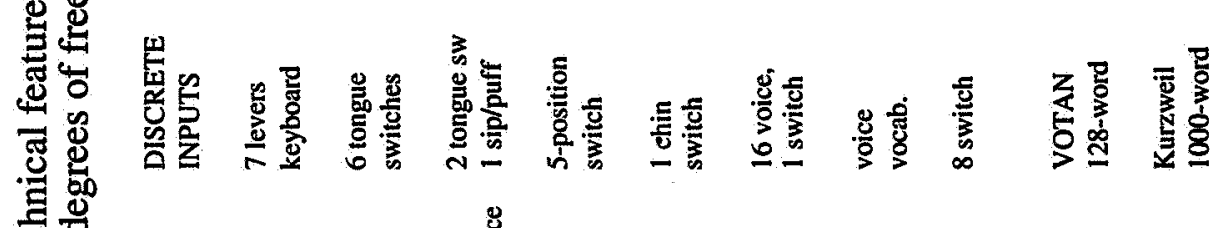

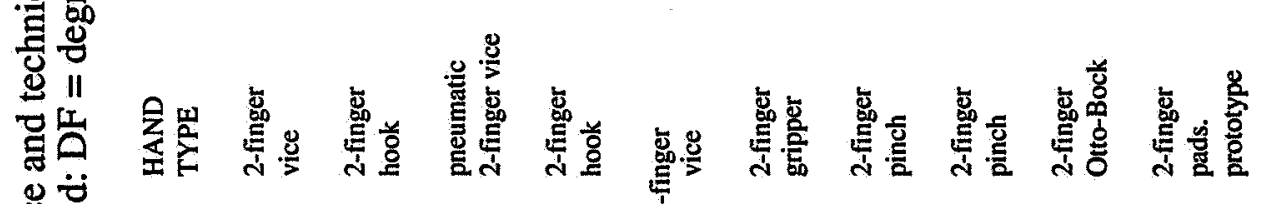

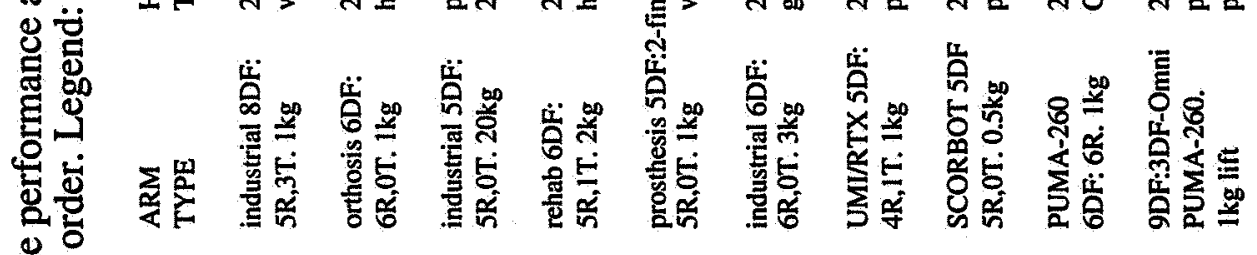

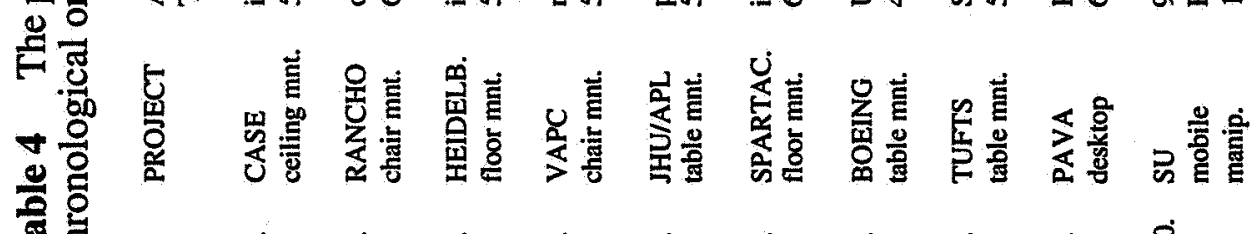
商 



\section{VIRTUAL ENVIRONMENTS}

$6 y+7$

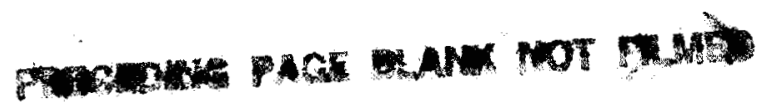

PAGE 60 INTENTIOUALY DLAW 



\title{
CREATING BETTER VIRTUAL WORLDS
}

\author{
Thomas A. Furness, III \\ University of Washington \\ Seattle, Washington
}

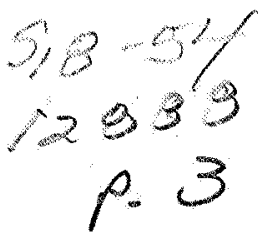

\section{INTRODUCTION}

Computing machines have brought new opportunities as well as challenges to our information age. While computer capacities and speeds have increased remarkably, our ability to communicate with these information engines is still limited by poor interfaces between the human and the computer. To harness and exploit the power of the computer as a tool and extender of our intellect, it is essential that we build new interfaces which are intuitive, transparent and take advantage of our innate spatial abilities.

The new technology of virtual interfaces provides a revolutionary way to improve the coupling of the human to the computing machine. Virtual controls and displays allow panoramic presentations in three dimensions to be made to the eyes, ears and hands of the user by projecting "virtual" images into the senses. To gain this global experience "the human literally wears the computer." Virtual interfaces transfer stimuli to the eyes, ears and hands, and use head, eye, hand movement and speech to control the machine. In this way the operator can be surrounded by a "circumambience" of computer synthesized information which becomes a spatial world through which he and machine can effectively communicate. The operator, in turn, interacts with his global medium by looking at objects, pointing his hands and giving verbal commands. The medium also allows virtual objects, which appear to be real but which are virtual projections, to be touched and manipulated by the hands. In order to create the virtual space representation of information, the virtual display hardware components are programmed with "mindware." Mindware is a special class of software that takes into account the perceptual organization of the human and dynamically creates three-dimensional sound, video and tactile images which appear as an artificial reality surrounding the user.

The virtual interfaces described above not only solve many existing interface problems but empower new and novel interfaces for operators in aerospace vehicles and other applications such as teleoperation, computer-aided design, medical imaging, prostheses for the handicapped and entertainment. Even though some aspects of virtual interface technology have been under development for two decades, there are still several key problems which must be addressed and resolved to make these concepts practical for research, industrial and consumer applications. These problems are summarized below: 


\section{THE PROBLEMS}

1. We don't understand the human factors of virtual space.

2. We don't know how to measure the "goodness" of virtual worlds.

3. Except for the more expensive military versions, virtual displays lack sufficient resolution for wide field-of-view presentations.

4. Position sensing technology (for control and image stabilization) needs improvement to increase precision and update rate while reducing noise and sensitivity to conductive materials.

5. Current graphics engines have limited update rate and polygon count.

6. The headgear is too heavy.

7. There are too many wires.

8. There is a need for a universal software architecture and tool kit for supporting future development and application of virtual worlds.

9. Virtual interfaces are too expensive.

10. There is no forum currently for discussing the development and applications of virtual worlds.

\section{POSSIBLE SOLUTIONS}

As research and development laboratories in universities and industry respond to the growing excitement about virtual worlds, we would propose that a research agenda be established to solve the problems above. Here are recommended approaches:

1. Establish a national knowledge base regarding the ergonomics, technology and application of virtual interfaces. Build a centralized literature collection, and interface with the Crew System Ergonomics Information Center (CSERIAC) at Wright-Patterson AFB, OH. Add to the Handbook of Perception and Human Performance and the Engineering Data Compendium published by the Air Force. Begin a scholarly journal to address issues of virtual interfaces including the psychophysics, technologies and applications of virtual worlds.

2. Explore new psychophysical and neurophysiological measurement techniques which operate within virtual worlds.

3. Develop high resolution parallel image projection approaches which reduce bandwidth and don't require the production of an aerial image. 
4. Develop position measurement approaches which are passive.

5. Improve image stabilization (close loop outside graphics processor). Develop graphics processor to support parallel information input.

6. Eliminate wires with passive sensing and data links.

7 Create microscanner for Maxwellian view image projection.

8. Create mindware to take into account the perceptual organization of the human. Standardize on network protocols for televirtuality and transportability of software and applications.

9. Establish a special interest group on virtual interface technologies under the aegis of the ACM, IEEE or HFS.

\section{CONCLUSIONS}

Virtual interfaces provide a bold new opportunity for solving many of the perplexing problems of interfacing human and machine intelligences. As pioneers, we are obligated to pursue the development of virtual interface technologies in a systematic way and to leave a technology base and tools as our legacy for others to build upon. If developed systematically, virtual interfaces can be one of the greatest advances of our age. 


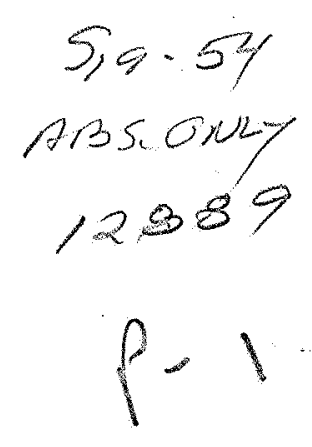

\title{
N95- 14032
} INTERACTING WITH VIRTUAL WORLDS

\author{
David Zeltzer \\ Massachusetts Institute of Technology \\ Cambridge, Massachusetts
}

What tools should we provide the user for defining and interacting with objects and agents in virtual environments at varying levels of complexity? Understanding the appropriate simplifications to make is critical for modeling non-trivial environments and agents with varying levels of autonomy. I describe a set of abstraction mechanisms appropriate for constructing and interacting with virtual worlds, and I discuss how programming, and direct manipulation or guiding techniques, can be used to afford users interactive access to graphical simulations at appropriate levels of abstraction.

Structural abstractions provide a mechanism for describing the kinematic structure of objects, such as transformation hierarchy for a jointed figure, and for defining physical attributes of objects, e.g., mass, stiffness, etc.

Procedural abstractions are a mechanism for defining processes that control rigid and non-rigid object motion, such as elastic deformation, collision detection, forward dynamics or inverse kinematics, independent of the structure of the agen or object.

Functional abstractions are the means with which we associate procedures with objects and object sub-assemblies, for the purpose of defining meaningful behaviors. This allows complex, largely unmanageable systems with many DOFs - like the human figure, with over 200 DOFs-to be decomposed into a set of small, manageable subsystems, each with few DOFs.

An agent consists of a structural definition, a set of functional units which define the behavior repertoire of that entity, and some means for selecting and sequencing (possibly concurrent) behaviors. The mechanism for selecting and sequencing these motor skills must link action with perception.

The lowest layer of abstraction is represented by objects and structures with no procedural attachment, and is called the machine level. At the other extreme, the task level, guiding and programming tools interact with agents through their repertoire of behaviors. Task level interaction emerges when guiding and programming techniques are applied at higher layers of abstraction.

Guiding and programming tools have a dual nature: they can be used to construct and modify instances of any of the abstraction types; or they can be used at runtime as control instruments. I describe how programming and guiding can be used with each of the four classes of abstraction. 


\section{VIRTUAL REALITY AT WORK}

Frederick P. Brooks, Jr. University of North Carolina

Chapel Hill, North Carolina

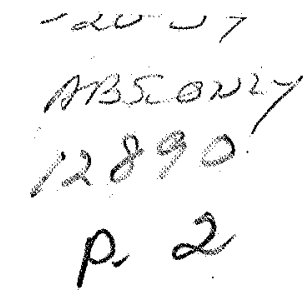

\section{INTRODUCTION}

The utility of virtual reality computer graphics in telepresence applications is not hard to grasp and promises to be great. When the virtual world is entirely synthetic, as opposed to real but remote, the utlility is harder to establish. Vehicle simulators for aircraft, vessels, and motor vehicles are proving their worth every day. Entertainment applications such as Disneyworld's StarTours are technologically elegant, good fun, and economically viable. Nevertheless, some of us have no real desire to spend our lifework serving the entertainment craze of our sick culture; we want to see this exciting technology put to work in medicine and science.

\section{TESTING A FORCE DISPLAY FOR SCIENTIFIC VISUALIZATION: MOLECULAR DOCKING (Ming Ouh-Young)}

2.1 The technology. This test uses a Model E-3 Argonne Remote Manipulator (the ARM) that offers a full 6-D input position sensing and full 6-D force and torque output. A Sun 4 provides computational power and ARM control; and E.\&S. PS-300 provides the visual output. A Tektronix stereo plate is used with passive circular polarizing glasses.

2.2 The molecular docking application. The enzyme dihydrofolate reductase binds to a variety of inhibitors, natural and synthetic. Of twelve inhibitors studied by our collaborators at Burroughs-Wellcome, the precise structure of the enzyme-inhibitor complex is known for six.

Binding energy is a complex function of atomic positions, involving Coulomb forces, van der Waals forces, nuclear forces, hydrogen-bonding forces, and thermodynamic forces. With 6 degrees of freedom in the drug docking, and up to 12 twistable bonds in the drugs, the docker is attempting to find a global minimum of the energy in a space of up to 18 dimensions, a space pock-marked with local minima. Brute-force algorithmic methods would require years of computer time to explore the space.

It is hard to imagine a visual display that would effectively produce all the needed insight to enable a user to manually dock the drug so as to minimize energy. We postulated that feeling the forces, particularly the $(1 / \mathrm{r})^{* *} 7$ and $(1 / \mathrm{r})^{* *} 13$ forces that define the receptor pocket, would substantially help chemists in manual docking.

2.3 The experimental results. Chemists can dock an inhibitor in about 25 minutes using our force display system. Ming's controlled experiments indicate an upper bound of about a factor of two improvement in docking performance when the visual display is aided with our force display. Our force display has many primitive characteristics, so there is hope for some improvement in this number, but an order-of-magnitude improvement doesn't seem to be in the cards. 
2.4 Whither now? We plan to get some actual drug-design experience with real drug designers. We also want to build or buy a finger-scale, versus arm-scale, 6-D force output device. The literature suggests that resolution should be at least as good with the scaled-down device, and stability and ease of use much better.

\section{TESTING A HEAD-MOUNTED DISPLAY FOR SCIENTIFIC AND MEDICAL VISUALIZATION (James Chung)}

3.1 The technology. We use the standard available technology: tiny TVs and Polhemus trackers. Our PixelPlanes graphics engine is faster than almost all of today's delivered engines. The Polhemus has respectable frame-rate but unacceptable lag for head-mounted displays. We measure $250-400 \mathrm{msec}$., and lag seems to depend upon source-sensor distance. We are pursuing several new tracker technologies.

3.2 The only advantage of head-mounted displays - intuitive navigation. Considered cold-bloodedly, today's head-mounted displays have many disadvantages: resolution, sharpness, field-of-view. Their inherent comparative advantage is intuitive navigation of the virtual space. It should be much easier for the user to know where he is and what he is looking at than in through-thewindow graphics. How much is this worth? How much does it improve performance in a real application?

3.3 The radiation-treatment planning application. Radiation oncologists have to plan the shape, intensity, and direction of multiple beams of radiation so as to burn the tumor without burning other sensitive or vital organs. This task requires imaginative use of 3-space, a good image of the anatomy of the patient, and, ideally, real-time calculation of the dose to the tumor and to unwanted organs.

3.4 The planned tests. We have volume-visualized MRI anatomical data on tumor patients. Our collaborating radiologists, in a controlled experiment, will devise treatment plans using the head-mounted display versus through-the-window graphics. We will measure task time and goodness of plan as experimental variables. 


\title{
USING VIRTUAL REALITY AS A TOOL TO STUDY THE RELATIONSHIP
} BETWEEN HUMAN BEINGS AND PHYSICAL REALITY

\author{
J. Lanier \\ VPL Research, Inc. \\ Redwood City, California
}

This presentation focused on the following areas:

1. VPL's Virtual Reality system (the RB2); What it is and what has been learned from it.

2. Unsolved technical problems in Virtual Reality.

3. Cultural, philosophical, and political inquiries demanded by. Virtual Reality.

4. The upcoming Reality Net project. 


\title{
THE AMES VIRTUAL ENVIRONMENT WORKSTATION: IMPLEMENTATION ISSUES AND REQUIREMENTS
}

\author{
$p, y$ \\ S. Fisher, R. Jacoby, S. Bryson, P. Stone, I. McDowall, M. Bolas, D. Dasaro, E. Wenzel, \\ C. Coler, and D. Kerr \\ NASA Ames Research Center \\ Moffett Field, California
}

This presentation describes recent developments in the implementation of a Virtual Environment Workstation in the Aerospace Human Factors Research Division of NASA's Ames Research Center. Introductory discussions are presented on the primary research objectives and applications of the system and on the system's current hardware and software configuration. Principal attention is then focused on unique issues and problems encountered in the workstation's development with emphasis on its ability to meet original design specifications for computational and graphics performance and for associated human factors requirements necessary to provide compelling sense of presence and efficient interaction in the virtual environment.

\section{Research Objectives and Applications}

In the Aerospace Human Factors Research Division of NASA's Ames Research Center, an interactive Virtual Environment Workstation (VIEW) has been developed to aid in design, simulation and evaluation of advanced data display and management concepts for operator interface design. The VIEW system provides a virtual auditory and stereoscopic image surround that is responsive to inputs from the operator's position, voice and gestures. As a low-cost, multipurpose personal simulation device, this variable interface configuration allows an operator to virtually explore a 360-degree synthesized or remotely sensed environment and viscerally interact with its components.

At present, the major thrust of this research effort centers on the development of guidelines and requirements that are necessary for implementation of virtual environments that are matched as closely as possible to human cognitive and sensory capabilities and that feature transparent, natural system interaction. It is projected that design specifications derived from this prototype system can help define the range of necessary and sufficient hardware and software architectures for future virtual environment systems.

Application areas of the virtual environment research are focused in:

TELEPRESENCE: Development of workstations for complex operational tasks such as telerobotic and telepresence control of remotely operated robotic devices and vehicles that require a sufficient quantity and quality of sensory feedback to approximate actural presence at the task site.

DATASPACE: Development of concepts and guidelines for "portable" multi-modal information management systems such as EVA Spacesuit visor display, with subsequent development of workstations for supervision and management of large-scale integrated information systems in which data manipulation, storage and retrieval, and system monitoring tasks can be spatially organized. 
VISUALIZATION: Design and evaluation of virtual environments to facilitate visualization of complex, three-dimensional data structures in areas such as architecture, engineering and computational fluid dynamics, and for interactive training or planning environments such as surgical simulation.

\section{VIEW System Configuration: Performance requirements and implementation}

The current Virtual Interface Environment Workstation consists of: a wide-angle stereoscopic display unit, glove-like devices for multiple degree-of-freedom tactile input, connected speech recognition technology, gesture tracking devices, 3D auditory display and speech-synthesis technology, and real-time computer graphic and video image generation equipment. This hardware is integrated with a realtime Unix workstation that supports the computations required to drive an external high-performance realtime 3D graphics system, processes input from up to 12 realtime input peripherals (e.g., the trackers and gloves), and provides other realtime task processing. A collection of software called the "simulation framework" has also been developed that consists of a well-documented library of functions to provide access to all of the system peripherals and software services, and of a collection of source files and simulation software that demonstrates the use of the major hardware and software components that make up the VIEW system in order to facilitate system reconfiguration for changing research requirements.

Before the beginning of the implementation phase of the Virtual Environment Workstation, a number of performance requirements and specifications for the major VIEW systems components were determined. These are discussed with respect to attaining an overall performance objective of providing $640 \times 480$ pixel resolution imagery at 30 frames per second over dual, independent, synchronized display channels with image viewpoints updated in coordination with head and limb motion.

\section{VIEW Implementation: Unique Issues and Problems}

The VIEW project attempts to match media and computational technology as closely as possible to the perceptual and cognitive capabilities of the human operator in order to achieve a state of Telepresence in which a sufficient quantity and quality of sensory feedback is presented to the operator to approximate presence at a remote task site or in a synthetic environment. The factors that directly influence and effect the achievement of this display configuration are divided into three main areas and are discussed in detail as examples of problems and challenges for the development of virtual environment systems. 


\subsection{Computational issues.}

- Problems in achieving VIEW objective of $30 \mathrm{~Hz}$ frame rate:

- Overall cumulative system transport delay

- "Realtime" UNIX

- Communication timing problems and scheduling artifacts

- Problems with original performance benchmarking

- I/O problems and requirements:

- RS232 requirements and host modifications

- Parallel communication between host and graphics system

- Floating point accuracy requirements in database representation.

- System calibration requirements for research applications (tracker, etc.)

- The need for evolution to object-oriented software structure for VIEW.

3.2 Graphics implementation issues:

- Requirements and problems in synching dual channel graphics systems for proper stereocopic image representation:

- SGI IRIS graphics

- HP SRX/TurboSRX graphics

- ISG graphics

- Differences between human eye and virtual camera image geometry:

- Viewport specification

- Perspective transformations (world rotation)

- Field of View specification

- Display calibration requirements

- Matrix/Euler/Quaternian representation; difficulties and advantages.

- Graphics language implementations and extensions (GOSAMR)

- Object editor

- Problems with animating textures

- Needed extensions

- Idiosyncrasies of lighting model in VIEW environment.

- Software structure for 3D information windows in VIEW environment: differences between screen space and world space.

3.3 Human Factors issues (Telepresence requirements):

- Guidelines and requirements necessary to provide stereoscopic images that are geometrically correct representations of the depth relationships and perspective of a rendered or captured scene are discussed (orthostereoscopy). For example, in an orthostereoscopic scene, an object in the Virtual Environment would subtend the same angle of visual field as the object it represents in the real world and would also impart correct motion parallax and motion perspective cues in relation to its virtual surroundings as the viewer changes position. 
- Differences between human eye and virtual camera image geometry are discussed with reference to view representation, depth of field representation, image separation for stereoscopic fusion, window effect in stereoscopic image fusion, foveal image display, and correct focal length and viewport specification.

- Differences between human eye and virtual camera image QUALITY such as luminance, resolution, color, and texture are discussed as well as differences between human visual system and virtual camera image DYNAMICS such as frame rate and image generation lag, axis of rotation for changing viewpoint (tracker offset), image and spatial distortions from non-parallel optical axes, and infinity optics versus variable accomodation performance.

- Multisensory virtual representations provide an additional dimension to the telepresence experience and are discussed in relation to synchronization with visual imagery, auditory localization and 3D sound cues, tactile feedback, and visceral simulation with motion platforms.

\section{References}

Fisher, Scott S. (1968) "Telepresence Master Glove Controller for Dexterour Robotic End-Effectors," Advances in Intelligent Robotics Systems, D. P. Casasent, Editor, Proc. SPIE 726, 1986.

Fisher, S. S., McGreevy, M., Humphries, J., Robinett, W., (1968) "Virtual Environment Display System," ACM 1986 Workshop on 3D Interactive Graphics, Chapel Hill, North Carolina. October 23-24, 1986.

Fisher, S. S. , McGreevy, M., Humphries, J., Robinett, W. (1987) "Virtual Interface Environment for Telepresence Applications," J. D. Berger, Editor, Proceedings of ANS International Topical Meeting on Remote Systems and Robotics in Hostile Environments, 1987.

Fisher, S. S., Wenzel, E. M., Coler, C., McGreevy, M. W., (1988) "Virtual Interface Environment Workstations," Proceedings of the Human Factor Society 32nd Annual Meeting (October 24-28, 1988, Anaheim, California)

Foley, James D. (1987), Interfaces for Advanced Computing. Scientific American, 257 (4), 126-135. 


\title{
VIRTUAL ACOUSTIC DISPLAYS
}

\author{
Elizabeth M. Wenzel \\ NASA Ames Research Center \\ Moffett Field, California
}

As with most research in information displays, virtual displays have generally emphasized visual information. Many investigators, however, have pointed out the importance of the auditory system as an alternative or supplementary information channel (e.g., Deatherage, 1972; Doll, et. al., 1986; Patterson, 1982; Caver, 1986). A three-dimensional auditory display can potentially enhance information transfer by combining directional and iconic information in a quite naturalistic representation of dynamic objects in the interface. Borrowing a term from Gaver (1986), an obvious aspect of "everyday listening" is the fact that we live and listen in a three-dimensional world. Indeed, a primary advantage of the auditory system is that it allows us to monitor and identify sources of information from all possible locations, not just the direction of gaze. This feature would be especially useful in an application that is inherently spatial, such as an air traffic control display for the tower or cockpit. A further advantage of the binaural system, often referred to as the "cocktail party effect" (Cherry, 1953), is that it improves the intelligibility of sources in noise and assists in the segregation of multiple sound sources. This effect could be critical in applications involving encoded nonspeech messages as in scientific "visualization," the acoustic representation of multi-dimensional data (e.g., Bly, 1982) and the development of alternative interfaces for the visually-impaired (Edwards, 1989; Loomis, et. al., 1990). Another aspect of auditory spatial cues is that, in conjunction with other modalities, it can act as a potentiator of information in the display. For example, visual and auditory cues together can reinforce the information content of the display and provide a greater sense of presence or realism in a manner not readily achievable by either modality alone (Colquhoun, 1975; Warren, et. al., 1981; O'Leary and Rhodes, 1984). This phenomenon will be particularly useful in telepresence applications, such as advanced teleconferencing environments, shared electronic workspaces, and monitoring telerobotic activities in remote or hazardous situations. Thus, the combination of direct spatial cues with good principles of iconic design could provide an extremely powerful and information-rich display which is also quite easy to use.

This type of display could be realized with an array of real sound sources or loudspeakers (Doll, et. al., 1986; Calhoun, et. al, 1987). An alternative approach, recently developed at NASA-Ames, generates externalized, three-dimensional sound cues over headphones in realtime using digital signal-processing (Wenzel, et. al., 1988a). Here, the synthesis technique involves the digital generation of stimuli using Head-Related Transfer Functions (HRTFs) measured in the two ear-canals of individual subjects (see Wightman and Kistler, 1989a:. Up to four moving or static sources can be simulated in a head-stable environment by digital filtering of arbitrary signals with the appropriate HRTFs. This type of presentation system is desirable because it allows complete control over the acoustic waveforms delivered to the two ears and the ability to interact dynamically with the virtual display. Other similar approaches include an analog system developed by Loomis, et. al. (1990) and digital systems which make use of transforms derived from normative mannikins and simulations of room acoustics (Genuit, 1986; Posselt, et. al., 1986; Persterer, 1989; Lehnert and Blauert, 1989). 
Such an interface also requires the careful psychophysical evaluation of listeners' ability to accurately localize the virtual or synthetic sound sources. For example, a recent study by Wightman and Kistler (1988b) confirmed the perceptual adequacy of the basic technique for static sources; source azimuth was synthesized nearly perfectly for all listeners while source elevation was somewhat less well-defined in the headphone conditions.

From an applied standpoint ; measurement of each potential listener's HRTFs may not be possible in practice. It may also be the case that the user of such a display will not have the opportunity for extensive training. Thus, a critical research issue for virtual acoustic displays is the degree to which the general population of listeners can obtain adequate localization cues from stimuli based on non-individualized transforms. Preliminary data (Wenzel, et. al., 1988b) suggest that using non-listener-specific transforms to achieve synthesis of localized cues is at least feasible.

For experienced listeners, localization performance was only slightly degraded compared to a subject's inherent ability, even for the less robust elevation cues, as long as the transforms were derived from what one might call a "good" localizer. Further, the fact that individual differences in performance, particularly for elevation, could be traced to acoustical idiosyncracies in the stimulus suggests that it may eventually be possible to create a set or "universal transforms" by appropriate averaging (Genuit, 1986) and data reduction techniques (e.g., principal components analysis), or perhaps even enhancing the spectra of empirically-derived transfer functions (Durlach and Pang, 1986).

Alternatively, even inexperienced listeners may be able to adapt to a particular set of HRTFs as long as they provide adequate cues for localization. A reasonable approach is to use the HRTFs from a subject whose measurements have been "behaviorally-calibrated" and are thus correlated with known perceptual ability in both free-field and headphone conditions. In a recently completed study, sixteen inexperienced listeners judged the apparent spatial location of sources presented over loudspeakers in the free-field or over headphones. The headphone stimuli were generated digitally using HRTFs measured in the ear canals of a representative subject (a "good localizer") from Wightman and Kistler (1988a,b). For twelve of the subjects, localization performance was quite good, with judgements for the non-individualized stimuli nearly identical to those in the free-field.

In general, these data suggest that most listeners can obtain useful directional information from an auditory display without requiring the use of individually-tailored HRTFs. However, a caveat is important here. The results described above are based on analyses in which errors due to front/back confusions were resolved. For free-field versus simulated free-field stimuli, experienced listeners exhibit front/back confusion rates of about $5 \mathrm{vs.} 10 \%$ and inexperienced listeners show average rates of about 22 vs. $34 \%$. Although the reason for such confusions is not completely understood, they are probably due in large part to the static nature of the stimulus and the ambiguity resulting from the socalled cone of confusion (see Blauert, 1983). Several stimulus characteristics may help to minimize these errors. For example, the addition of dynamic cues correlated with head-motion and wellcontrolled environmental cues derived from models of room acoustics may improve the ability to resolve these ambiguities. 


\section{REFERENCES}

Blanert, J. (1983) Spatial Hearing. The MIT Press: Cambridge, MA.

Bly, S. (1982) Sound and computer information presentation. Unpublished doctoral thesis (UCCRL53282) Lawrence Livermore National Laboratory and University of California, Davis, CA.

Calhoun, G. L., Valencia, G., and Furness, T. A. III (1987) Three-dimensional auditory cue simulation for crew station design/evaluation. Proc. Hum. Fac. Soc., 31, 1398-1402.

Cherry, E. C. (1953) Some experiments on the recognition of speech with one and two ears. $J$. Acoust. Soc. Am., 22, 61-62.

Colquhoun, W. P. (1975) Evaluation of auditory, visual, and dual-mode displays for prolonged sonar monitoring in repeated sessions. Hum. Fact., 17, 425-437.

Deatherage, B. H. (1972) Auditory and other sensory forms of information presentation. In H.P. Van Cott and R.G. Kincade (Eds.), Human Engineering Guide to Equipment Design, (rev. ed.), Washington, DC: U.S. Government Printing Office, 123-160.

Doll, T. J., Gerth, J. M., Engelman, W. R. and Folds, D. J. (1986) Development of simulated directional audio for cockpit applications. USAF Report No. AAMRL-TR-86-014.

Durlach, N. I. and Pang, X. D. (1986) Interaural magnification. J. Acoust. Soc. Am., 80, 1849-1850.

Edwards, A. D. N. (1989) Soundtrack: An auditory interface for blind users. Hum. Comp. Interact., 4, 45-66.

Gaver, W. (1986) Auditory icons: Using sound in computer interfaces. Hum. Comp. Interact., 2, 167-177.

Genuit, K. (1986) A description of the human outer ear transfer function by elements of communication theory. Proc. 12th ICA (Toronto), Paper B6-8.

Lehnert, H. and Blauert, J. (1989) A concept for binaural room simulation. ASSP Workshop on. Applications of Signal Processing to Audio and Acoustics, New Paltz, NY.

Loomis, J. M., Herbert, C., and Cicinelli, J. G. (1989) Active localization of virtual sound sources. Submitted to J. Acoust. Soc. Am.

O'Leary, A. and Rhodes, G. (1984) Cross-modal effects on visual and auditory object perception. Perc. \& Pychophys., 35, 565-569.

Patterson, R. R. (1982) Guidelines for Auditory Warning Systems on Civil Aircraft. Civil Aviation Authority Paper No. 82017, London. 
Persterer, A. (1989) A very high performance digital audio signal processing system. ASSP Workshop on Applications of Signal Processing to Audio and Acoustics, New Paltz, NY.

Posselt, C., Schroter, J., Opitz, M., Divenyi, P., and Blauert, J. (1986) Generation of binaural signals for research and home entertainment. Proc. 12th ICA (Toronto), Paper B1-6.

Warren, D. H., Welch, R. B., and McCarthy, T. J. (1981) The role of visual-auditory "compellingness" in the ventriloquism effect: Implications for transitivity among the spatial senses. Perc. \& Psychophys., 30, 557-564.

Wenzel, E. M., Wightman, F. L., and Foster, S. H. (1988a) A virtual display system for conveying three-dimensional acoustic information. Proc. Hum. Fac. Soc., 32, 86-90.

Wenzel, E. M., Wightman, F. L., Kistler, D. J., and Foster, S. H. (1988b) Acoustic origins of individual differences in sound localization behavior. J. Acoust. Soc. Amer., 84, 579.

Wightman, F. L. and Kistler, D. J. (1989a) Headphone simulation of free-field listening I: stimulus synthesis. J. Acoust. Soc. Amer., 85, 858-867.

Wightman, F. L. and Kistler, D. J. (1989b) Headphone simulation of free-field listening II: psychophysical validation. J. Acoust. Soc. Amer., 85, 868-878. 


\title{
VARIETIES OF VIRTUALIZATION
}

\author{
Stephen R. Ellis \\ NASA Ames Research Center \\ Moffett Field, CA \\ and \\ University of California \\ Berkeley, California
}

Natural environments have a content, i.e., the objects in them; a geometry, i.e., a pattern of rules for positioning and displacing the objects; and a dynamics, i.e., a system of rules describing the effects of forces acting on the objects. Human interaction with most common natural environments has been optimized by centuries of evolution. Virtual environments created through the human-computer interface similarly have a content, geometry, and dynamics, but the arbitrary character of the computer simulation creating them does not insure that human interaction with these virtual environments will be natural. The interaction, indeed, could be supernatural but it also could be impossible.

An important determinant of the comprehensibility of a virtual environment is the correspondence between the environmental frames of reference and those associated with the control of environmental objects. The effects of rotation and displacement of control frames of reference with respect to corresponding environmental references differ depending upon whether perceptual judgement or manual tracking performance is measured.

The perceptual effects of frame of reference displacement may be analyzed in terms of distortions in the process or virtualizing the synthetic environmental space. The effects of frame of reference displacement and rotation have been studied by asking subjects to estimate exocentric direction in a virtual space. Exocentric judgements involve the estimation of the orientation of one external object with respect to an external frame of reference. They may be contrasted with egocentric judgements in which objects orientation is judged with respect to the viewer himself. Though alternative models of the errors of exocentric direction may be compared, the most parsimonious explanation may be that viewers misjudge the direction of the view vector used to produce the display. The motor effects of frame of reference misalignment have been studied using a technique of three dimensional tracking in which a target moves irregularly in 3 axes. Pursuit tracking errors may be separated into linearly additive visual and visual-motor components, but the pattern of error as a function of angular misalignment may not generalize to compensatory tracking or to nonholonomic control modes. 


\section{REFERENCES}

Ellis, S. R., Kim, Won Soo, Tyler, Mitchell, McGreevy, M. W., and Stark, L. (Nov. 1985). Visual enhancements for perspective displays: Perspective parameters. Proceedings of the International Conference on Systems, Man and Cybernetics. IEEE Catalog \#85CH2253-3, 815-818.

Ellis, Stephen R., Grunwald, Arthur J., Smith, Stephen, and Tyler, Mitchell. (1988) Enhancement of man-machine communication: The human use of inhuman beings. Proceedings of IEEE CompCon88, February 29 - March 4, 1988, San Francisco, CA.

Grunwald, Arthur, and Ellis, Stephen R. (1986) Spatial orientation by familiarity cues. Training, human decision making, and control. J. Patrick and D. D. Duncan, eds., Elsevier, North Holland, 257-279.

Grunwald, Arthur, Ellis, Stephen R., and Smith, Stephen. (1988) Spatial orientation in pictorial displays. IEEE Trans. on Systems, Man and Cybernetics, 18, 425-436.

Kim, W. S., Ellis, S. R., Tyler, M., Hannaford, B., and Stark, L. (1987) A quantitative evaluation of perspective and stereoscopic displays in three-axis manual tracking tasks. IEEE Trans. on Systems, Man and Cybernetics, SMC-17, 61-71.8. 


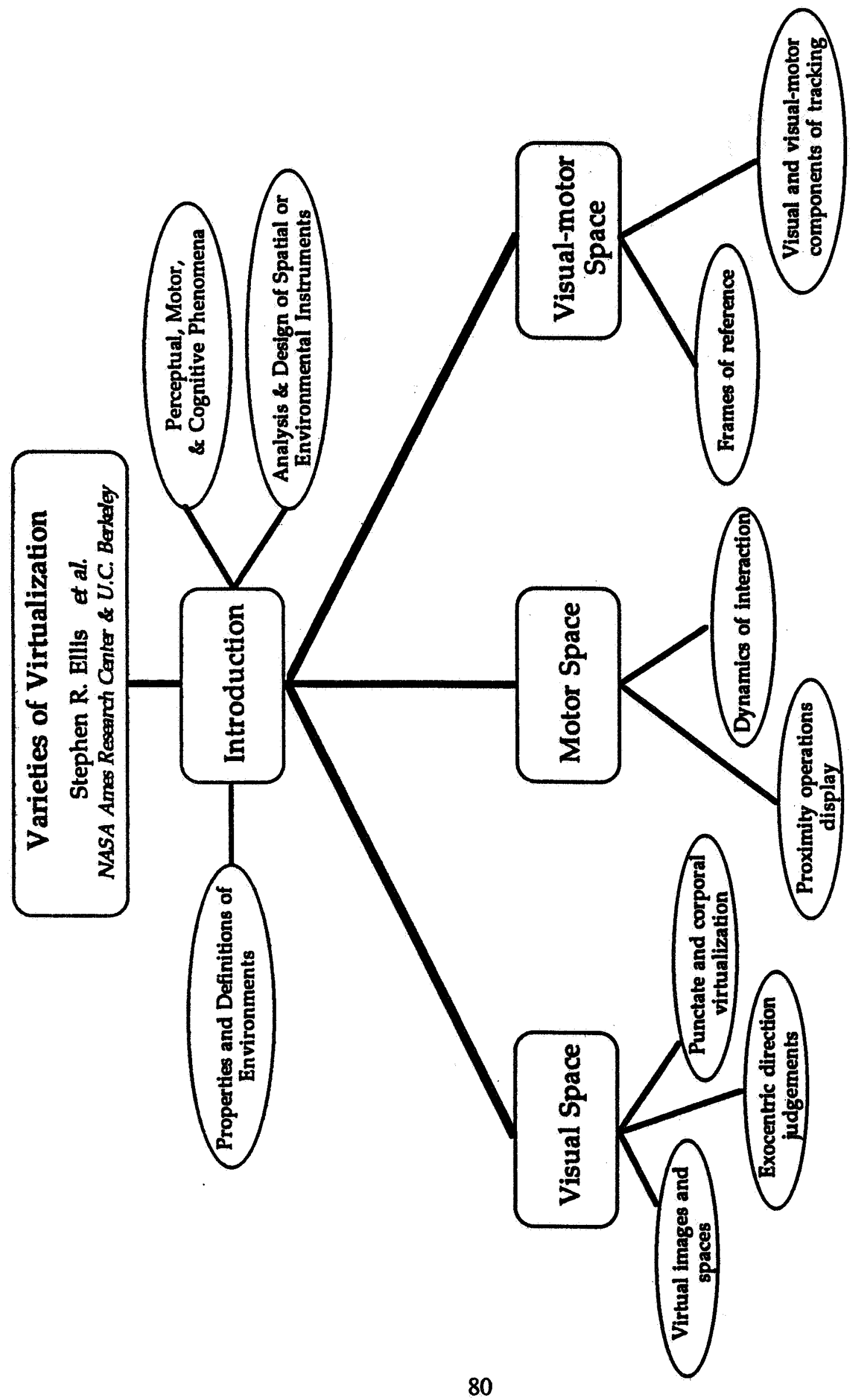




\title{
THE EXPLORATION METAPHOR
}

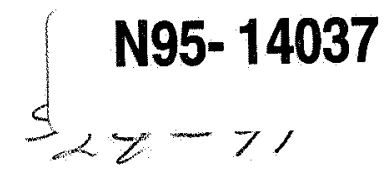

\author{
Michael W. McGreevy \\ NASA Ames Research Center \\ Moffett Field, California
}

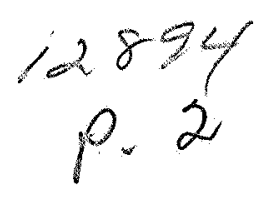

NASA's experience in planetary exploration has demonstrated that the desktop workstation is inadequate for many visualization situations. The primary mission displays for the unmanned Surveyor missions to the moon during the mid-1960s, for example, were environmental images assembled on the inside surfaces of spherical shells. Individual images taken in 1971 by the first Mars orbiter, Mariner 9, were applied to the outside of four-foot diameter spheres, creating composite photographs of the entire planet. During the Viking mission to Mars in 1976, initial orbiter photos were mosaicked on large tables or on walls to serve as group decision-making displays. The environments surrounding the two Viking landers were visualized using stereo and panoramic images, desktop models, and full-sized replicas of the lander and the surrounding rocks.

Future exploration missions will greatly benefit from advances in digital computer and display technology, but there remain unmet user interface needs. Unfortunately, the desktop workstation is generally considered to be the starting point of user-computer interface design, and most current human-computer interface research emphasizes the desktop metaphor. In this view, computing is thought to be comparable to paperwork, and the objects and functions available to the user reflect this emphasis. Point-and-click interaction with icons and windows is supportive of operations performed on text and simple pictures, but this approach is inadequate for planetary exploration.

Alternative user interfaces and metaphors are needed for planetary exploration and other interactions with complex spatial environments. These interfaces and metaphors would enable the user to directly explore environments and naturally manipulate objects in those environments. Personal simulators, virtual workstations, and telepresence user interfaces are systems capable of providing this integration of user space and task space. The Exploration Metaphor is a useful concept for guiding the design of user interfaces for virtual environments and telepresence. To apply the Exploration Metaphor is to assert that computing is like exploration, and to support objects, operations, and contexts comparable to those encountered in the exploration of natural environments. The Exploration Metaphor, under development for user interfaces in support of NASA's planetary exploration missions and goals, will also benefit other applications where complex spatial information must be visualized.

Visualization methods and systems for planetary exploration are becoming increasingly integrated and interactive as computing technology.improves. These advances will benefit from virtual environment and telepresence interface technology. A key development has been the processing of multiple images and other sensor data to create detailed digital models of the planets and moons. Data from images of the Earth, Mars, and Miranda (a moon of Uranus), for example, have been converted into three dimensional models, and dynamic virtual fly-overs have been computed as demonstrations. Similar processing of lower altitude photography and the use of computer aided design tools promise to produce very detailed models in the future. Currently, the Magellan spacecraft is 
more than half way to Venus on a mission to digitize the entire surface of that planet, and this, too, will provide an interactive digital model for virtual exploration on Earth.

Future plans call for multiple unmanned rovers to traverse the surface of Mars, enabling geoscientists located at a central Mars base to explore the planet via telepresence. In addition, Earth-bound mission operators and scientists will be able to access integrated digital models of Mars as the missions progress. The Exploration Metaphor will contribute to the design of user interfaces for these applications. 


\title{
MEASUREMENT AND MODIFICATION OF THE EEG AND RELATED BEHAVIOR
}

\author{
M. B. Sterman \\ University of California \\ Los Angeles, California \\ and \\ Veterans Administration Medical Center \\ Sepulveda, California
}

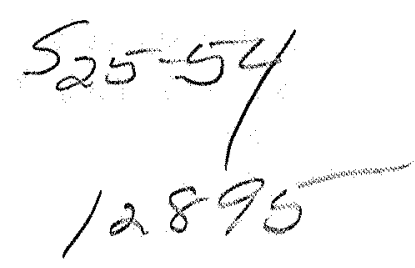

p 2

Early studies in our laboratory showed that cats could be trained to alter spontaneous EEG patterns through contingent food reinforcement of operant configurations. In particular, rhythmic EEG patterns in sensorimotor cortex, which were related behaviorally to motionlessness, were facilitated in this manner, and showed sustained alteration across states and time. Electrophysiological changes in the sensorimotor pathways were found to accompany this effect. Additionally, several striking behavioral changes were seen as well, including in particular an enhancement of sleep and an elevation of seizure threshold to epileptogenic agents.

This latter observation raised the possibility that human seizure disorders might be influenced therapeutically by similar training. However, in man the use of food reinforcement was not practical. Further, unlike our cats, human epileptics present with characteristic EEG abnormalities. An evaluation of these abnormalities from the perspective of our cat data showed that while normal humans had analogous patterns, untreated epileptics were often deficient in normal rhythmic sensorimotor activity and had EEG patterns dominated, instead, by slower and more poorly organized patterns. It thus became necessary to develop a very different strategy in applying this methodology to epileptics.

Our objective in human EEG feedback training became not only the facilitation of normal rhythmic patterns, but also the suppression of abnormal activity, thus requiring complex contingencies directed to the normalization of the sensorimotor EEG. To achieve this, a multicomponent frequency analysis was developed to extract and separate normal and abnormal elements of the EEG signal. Each of these elements was transduced to a specific component of a visual display system, and these were combined through logic circuits to present the subject with a symbolic display. This display allowed the subject to score "points" in a variety of game formats through the normalization of EEG characteristics. Variable criteria provided for the gradual shaping of EEG elements towards the desired normal pattern. Some $50-70 \%$ of patients with poorly controlled seizure disorders experienced therapeutic benefits from this approach in our laboratory, and subsequently in many other laboratories as well.

A more recent application of this approach to the modification of human brain function in our laboratory has been directed to the dichotomous problems of task overload and underload in the contemporary aviation environment. At least $70 \%$ of all aviation accidents have been attributed to 
the impact of these kinds of problems on crew performance. The use of the EEG in this context has required many technical innovations and the application of the latest advances in EEG signal analysis. Our first goal has been the identification of relevant EEG characteristics. Additionally, we have developed a portable recording and analysis system for application in this context. Findings from laboratory and in-flight studies suggest that we will, indeed, be able to detect appropriate changes in brain function, and feed this information to on-board computers for modification of mission requirements and/or crew status.

Supported by the Veterans Administration, the Public Health Service, NASA, and Northrop Aviation. 


\title{
THE "TELEPRASENZ" CONSORTIUM: STRUCTURE \& INTENTIONS
}

\author{
Jens Blauert \\ Ruhr-Universitat Bochum \\ Bochum, FR Germany
}

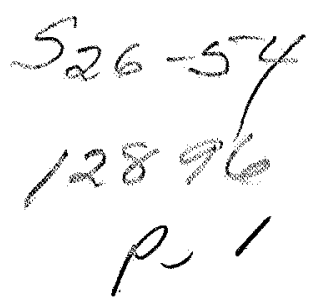

The Teleprasenz-Consortium is an open group of currently 37 scientists of different disciplines who devote a major part of their research activities to the foundations of telepresence technology. The necessary support for their work is provided by German federal and state sources as well as by European agencies and industry. The activities of the consortium are organized into three main branches: virtual environment, surveillance and control systems and speech and language technology. In the following, a brief summary of the main activities in these areas is given.

Telepresence technology - in this context - is basically understood as a means to bridge spatial and temporal gaps as well as certain kinds of concealment, inaccessibility and danger of exposure (e.g., microscopic dimensions, non-perceptibility by human senses, sensoric disabilities, speech and language problems, load of emissions).

Virtual Environment: In this group, it is the aim to provide a virtual environment for specific tasks such as telesurveillance or telecooperation applications. Although the main focus was directed on the auditory and visual components so far, future work will include tactile and kinesthetic cues.

Surveillance and Control Systems: This group is mainly engaged in tasks which concern control technology, diagnostics of complex systems in biology and technology as well as in accompanying problems in signal processing and software technology.

Speech and Language Technology: This group is concerned with several projects in the areas of automatic speech in- and output, speech coding and symbolic processing fore telepresence scenarios.

Current research proposals of the Teleprasenz Consortium include a telemanipulator system and a testbed for polysensory virtual environment as well as many smaller research and development contracts. An information brochure on Teleprasenz (in German) will be available at the conference. 


\title{
MACHINE PERCEPTION AND ITS ROLE IN VIRTUAL ENVIRONMENTS
}

opm?

\author{
S. K. Ganapathy \\ AT\&T Bell Laboratories \\ Holmdel, New Jersey
}

(No written material provided) 


\section{SUMMARIES OF PANEL DISCUSSIONS}





\title{
PANEL DISCUSSION ON TELEPRESENCE AND SENSORIMOTOR
}

\author{
ADAPTATION \\ R. B. Welch, Chair \\ NASA Ames Research Center \\ J. Loomis \\ University of California, Santa Barbara \\ R. Pepper \\ Science Applications International, Inc. \\ M. Kreuger \\ Artificial Reality Corporation \\ and \\ T. Etter \\ ANPA West
}

$8 \mathrm{mpr}$ to

Four questions were addressed. The following represents a synopsis of the thoughts of each seminar member (or members) with respect to his/her assigned item.

\section{What do we mean by the term "telepresence" and how can we measure it? (Jack Loomis)}

If virtual and remotely sensed environments are comprised of the same sensory cues as nearsensed worlds (i.e., "equivalent configurations"), telepresence will be complete. This rather self-evident observation leads to the much more interesting issue of why some everyday perceptual events are externalized (experienced as non-self), while others are not. Pain, for example, is perceived as internal, while most objects in our perceptual experience are externalized.

It has been proposed that the degree of experienced externalization is related to the range of variation in the efference to the motor system that results in some invariance in the sensory inflow (e.g., Brunswik, Gibson, Polanyi). According to this view, pain is completely internalized because the sensory signals are constant with changes in motor outflow. A positive afterimage produces intermediate externalization because the change in its angular position within the visual field is strictly correlated with eye movements. The most compelling sense of externalization arises when sensory input from all of the senses provides converging evidence, under active exploration, of some fixed entity. If this compelling externalization arises when using a virtual display or teleoperator, we say that there is "telepresence," but just as interesting is the externalization that occurs with ordinary near-sensing of the world.

Finally, a necessary condition for externalization (telepresence) when using a virtual display or teleoperator is that the observer be capable of internalizing the dynamics of the linkage (plant) 
mediating contact with the virtual or remote environment. If the linkage is too unnatural, arbitrary, or non-linear for this to occur, the observer will experience little externalization of the distal environment. Nevertheless, adaptation may serve to re-establish some sense of externalization. Once this happens, the plant model becomes transparent to the observer and externalization of the distal environment results. A common example involves long-cane training for the blind. At first, the trainee fumbles a lot and is very aware of the cane rather than the environment. As skill develops, however, manipulation of the cane becomes more automatic, resulting eventually in its transparency and in the externalization of the environment with which the tip of the cane is making contact.

\section{Of what good is telepresence? Does it contribute to performance? Is it self-evident that the more telepresence, the better? (Ross Pepper)}

A number of benefits result when the experience of telepresence is increased. First, under heightened telepresence, less operator training is necessary. Second, teleoperator performance under these conditions is more comparable to direct human performance. Third, optimal telepresence can be expected to increase the "emotionality" of the remote environment, depending on the content of the replicated sensory experience. An example of the latter point is the enjoyable visceral feelings of actually "being there" experienced by the viewers of a very realistic, action-filled movie. Finally, the very search for telepresence will stimulate the conceptual analysis of perceptual issues (see Loomis' remarks).

Telepresence contributes to performance, but not without certain costs. The benefits of telepresence to performance are that it (1) reduces errors and task performance variability, (2) increases precision, (3) reduces training costs, (4) enables performance similar to direct viewing conditions, and (5) results in greater adaptability to changing task conditions.

The costs of enhanced telepresence are (1) an increase in system complexity, (2) reduced reliability, (3) increased initial investment, and (4) increased maintenance costs.

It may be concluded that teleoperator system enhancements that produce marked increases in performance generally result in a heightened experience of telepresence. Examples of such enhancements are changing a monocular visual display to a stereoscopic display and providing force feedback for the manipulating hand.

3. Which characteristics of the teleoperator or virtual environment system contribute to telepresence, which are irrelevant, and which degrade it? (Myron Krueger and Tom Etter)

\section{(Krueger's remarks)}

A number of factors influencing telepresence or artificial worlds can be addressed, the most influential of which is maintaining a short delay between action and response. This is more important than a high sample rate by means of pipelining techniques. It is also claimed that short delays are more important than a realistic portrayal in graphic worlds. The allowable delay varies with the task. In particular, fine manipulation requires shorter delays than does gross manipulation. The ability to look around suffers with delays longer than .03 seconds; it can be done, but the operator is aware of the lag. In general, there is a decline in possible telepresence with distance. Beyond a rather short 
distance, the only way the immediacy of telepresence can be attained is by interacting locally with a graphic world.

Another dimension that may be explored is the effect of unencumbering sensors and actuators designed to increase telepresence. The first observation is that the presence of encumberance signals a distance from the world in which one wishes to feel present. Also, the discomfort caused by prolonged encumberance can lead to a net loss in telepresence.

It should also be observed that realism may not necessarily be the ultimate goal of telepresence. Natural behavior can be instantly achieved in 2-D realities which are easier to enter and exit than are 3-D worlds than can only be experienced by "suiting up." Furthermore, if artificial worlds are to be used for applied purposes, many traditional techniques will be demanded that violate realism. While people may initially obey the spatial metaphor to go from one point in a virtual world to another, later they will probably want to make the trip instantly by means of virtual teleportation.

A final event that will affect telepresence in artificial realities, as well as in tasks where two telerobots cooperate at a remote site, is the representation of the operator at the effector end. If the individual is to be represented, it is inevitable that a face must be provided and no method of threedimensional facial image capture and portrayal currently exists.

\section{(Etter's remarks)}

What enhances telepresence? Here's a related question: What enhances the "realism" of artificial food? In this case we're clearly dealing with two separate issues, taste and food value. I suggest that essentially the same two issues arise in the case of "artificial presence." We must distinguish the sense of presence from the physical component of presence, whatever exactly that may be. Suppose that you are wired into a computer-generated virtual environment. Does the computer supply this physical component of presence? Do the wires carry it?

Bell's theorem shows that any informational model of quantum phenomena (e.g., computer simulation) must contain faster-than light information paths, thereby contradicting the theory of relativity. Thus, if relativity does in fact govern the transmission of information, the connections among things in the world cannot be purely informational-they must also transmit something else. Let's call this something else the $\mathbf{L}$ factor.

The $\mathrm{L}$ factor involves negative probabilities which makes its meaning quite mysterious. However, its mathematics are very simple and it leads to an elegant and robust explanation of basic quantum theory. The only alternative to the $L$ factor is to assume the existence of hidden, non-local information paths. This is easy to interpret in familiar ways, but it fills the world with unobservable entities and generally creates a mess.

Assuming that we go with the $\mathrm{L}$ factor, what might this mean for telepresence? Could the $\mathrm{L}$ factor be the "food value" of physical presence? Could there be a long-term danger in going without it, like eating food with vitamins? With a more scientifically advanced technology, might we be able to build virtual environments that supply it? 


\section{To what extent is it necessary for the human operator to adapt to the teleoperation or vir- tual environment situation in order to experience a strong sense of telepresence and/or to function effectively? (Robert Welch)}

With current technology, remotely sensed and virtual worlds inevitably entail significant intersensory and sensorimotor discordances, which serve to degrade both performance and the experience of telepresence. However, it is likely that these problems can be overcome, at least partially, by means of adaptation or adaptation-like processes, of which there are four varieties: (1) intersensory bias, (2) perceptual adaptation, (3) perceptual-motor adaptation, and (4) habituation.

The most commonly cited example of intersensory bias is "visual capture"-the "dominance" of vision over felt limb position. It represents an immediate and very short-lived resolution of the intersensory conflict since it disappears as soon as vision is precluded. The magnitude of intersensory bias is inversely related to the size of the intersensory discrepancy and it is facilitated by familiarity with the intersensory relationship (e.g., a view of the hand) as well as by the presence of congruent intersensory characteristics of the stimulus object such as its felt and seen shape, motion, orientation, and size. These same factors are presumably involved in creating a compelling experience of telepresence.

Perceptual adaptation represents a compensatory recalibration of one or both of the sensory systems that serves to resolve the intersensory discrepancy. In contrast to intersensory bias, it persists as an aftereffect. This type of adaptation will not occur with very large discrepancies, such as 180-degree optical rotation.

Perceptual-motor adaptation refers to changes in the observer's performance. If perceptual adaptation has occurred, perceptual-motor adaptation is likely to follow suit, whereas the reverse does not always hold, particularly with very large rearrangements. For example, even though after several days of practice one will get quite good at operating in a 180-degree rotated visual world, it will continue to look upside down.

Factors that affect the magnitude of perceptual and/or perceptual-motor adaptation are active (versus passive) movement, immediate feedback, error correction, distributed practice, and incremental exposure. Presumably, these variables would similarly influence adaptation to rearranged remote or virtual environments.

After prolonged experience with the rearranged sensory environment, it will come to look familiar, a phenomenon referred to here as habituation. This may occur even when no perceptual adaptation has taken place. For example, well practices wearers of inverting goggles become so accustomed to their upside-down visual world that for periods of time they pay no attention to its orientation. It is likely that at those times the feeling of presence is strong and that after much practice the same would be true for a remote or virtual world that may have initially appeared quite discrepant or bizarre.

Finally, if and when it is possible to construct a teleoperator or virtual environment system that is completely accurate in its portrayal of the naturally sensed world, there will be little to which the operator will have to adapt. However, even with such an ideal system, some adaptation will be 
necessary to overcome the inter-operator differences in perception and reponse that have been widely reported. 


\section{PANEL DISCUSSION ON PERFORMANCE MEASUREMENT AND EVALUATION METHODS}

Daniel T. Ling, Chair (IBM), Ruzena Bajcsy (University of Pennsylvania), Anatal Bejczy (Johnson Propulsion Laboratory), Beth Marcus (Exos, Inc.), and Misha Pavel (Stanford University)

The seminar was introduced by Daniel Ling who presented a brief outline of a number of key issues which need to be addressed within the context of performance measurement and evaluation methods.

1. The context of any evaluation must be the task. How should the tasks be defined and decomposed?

2. What needs to be measured? This really means obtaining the data needed to develop models. Models are needed for both the human operator as well as the hardware/software system.

3. What criteria should be used for the measurements? This might include task performance (mean and variance), training required, ease of recall, "likeability" which may NOT correspond to any simple measure of performance, etc.

4. Need for rigorous experiments as well as general observations and rules of thumb.

Misha Pavel advocated evaluations based upon explicitly specified system models. He also discussed the importance of task analysis.

\section{Model Based Evaluation (Misha Pavel)}

Evaluation of interactive human-machine systems is usually very difficult because there are too many variables, too many conditions and the results obtained with one system are often difficult to apply to other similar ones (generalization problem). Frequently, the only way to make this problem manageable is to formulate an explicit "computational" model of all the components of the system including the human operator. Such a model, because of the constraints it imposes, will permit considerably more efficient evaluation. For example, many formal models specify a functional relationship among variables providing only a few free parameters. Then, instead of evaluating every possible combination of all the variables, one must only determine a smaller set of parameters.

Whenever performance of a system is measured, there is an underlying model even if it is not explicitly specified. These implicit models are usually weaker in that they impose fewer constraints. They are therefore more general and have less predictive power. The distinction between weak and strong models is useful. A strong model imposes strong constraints on the system behavior, and therefore allows for very efficienct evaluation. Its disadvantage is that it is likely to be incorrect. A strong model, for example, would have a second order polynomial relationship between two variables. A weak model would more likely be correct but would not allow as efficient evaluation. 
For example, a very weak model would only require a monotonic relationship between two variables. This model is less constraining, but makes less accurate predictions.

\section{Task Analysis}

Specification of a model has further advantage in that it requires a careful task analysis. The analysis alone can frequently reveal design problems before a prototype is actually built. For example, task analysis can reveal whether the systems objectives are well defined. Objectives are necessary in order to test and evaluate any system. Too often we see evaluation of a system with no particular objective specified to the operatory. The generalizability of such results is questionable because in real-world situations the operator always has a goal to accomplish.

In some cases, the system is designed for many purposes (e.g., a teleoperator for extraterrestrial applications) and it is difficult to specify a single task. In those situations one should estimate the envelope of the performance capabilities and analyze several extreme tasks. For example, in the case of a teleoperator design, one might expect tasks of grasping and moving large objects, executing a complex trajectory, and, at the other extreme, inserting a small fastener into a hole.

One of the results of careful task analysis is the determination of the variables to be measured. Such analysis might reveal the need to assess other measures than the usual duration of a given task. In particular, an important by-product of a task analysis is the notion that the operator usually has a choice of strategies. A typical, simple example is the speed-accuracy trade-off arising in evaluation of various input devices. In interactions between a human and a machine most tasks can be performed faster by sacrificing accuracy. A simple, weak model, of the speed-accuracy trade-off leads to the following evaluation principles:

1. Always measure both speed and accuracy.

2. Assure sufficient time pressure on the operator so that their performance has measurable errors.

The second principle assures that the experimenter can detect if the operator changes strategy between two conditions or two systems.

While I have indicated the need for model-based evaluation, it is important to note that using models can be very difficult. One argument frequently used against the model-based approach is that the validation of the model is by itself a complex empirical problem requiring too large an experimental effort to be justified. Although this is a serious problem the cost of evaluation must be compared to the savings from the ability to generalize the conclusions and to debug the system.

Ruzena Bajcsy echoes the theme of model based evaluation and task analysis. 


\section{Notes (Ruzena Bajesy)}

I am most interested in sensory system performance. The way I view performance evaluation is that it implies:

Models

By models, I mean characterization of the physics and geometry of the sensory system. Engineers have been doing modeling for a long time. For example, for a visual sensor we must model its optics, absorption functionl, resolution, signal to noise ratio, etc. Similarly for pressure/force sensors we should model sensitivity, saturation, hysteresis, and the like. For manipulators, the models give degrees of freedom, the reach-space, calibration parameters, repeatability, accuracy, etc. In other words, the models provice for each parameter the limits of performance. What is not so obvious is that similar modeling principles must apply to software modules as well as to hardware modules. Examples of these software modules are: convolution (parameter is width and orientation of the filter), edge-detector (looking for the first derivative or second derivative, the parameter is threshold of acceptance of the peak in the first derivative or second derivative), various fitting procedures to fit surfaces, volumes and so forth. This is also related to the choice of primitives which serve as data reduction mechanisms, and which are necessary for displays, and/or other communication means. These primitives are in fact more sophisticated "sensors" implemented in software.

\section{Models of the Task}

While models of the sensors tell us what CAN be measured and detected, a model of the task will tell lus what NEEDS to be measured. The bottom line is, that by the choice of models, one artificially constrains the problem, but this is the only way to have a predictable system where you take as much data as you NEED, determined by the TASK, and you explain the data/measurements as much as the models allow. So evaluation of a system is always in terms of some a priori chosen models.

Antal Bejczy described specific issues which need to be evaluated for teleoperator systems in space applications. He also emphasized task analysis and decomposition and an understanding of the target user.

\section{Measurement and Evaluation in Teleoperator Systems (Antal Bejczy)}

Virtual environments for teleoperator control will play an increasingly important role as the system and task complexity and telecommunication time delay increase. Preview and predictive graphics displays for task planning and time-delayed control execution can create an essentially virtual environment for the operator. When the graphics images of the manipulator and eventually also of elements of the task environment are overlayed over the real TV images of the manipulator and task environment, the performance capabilities of this human-machine interface technique will essentially depend on the fidelity of calibration by which the graphics images are related to the real images. This is an objective measure, and depends on the knowledge of a set of system parameters (TV camera parameters), on mathematical calibration techniques, and on the resolution of graphics images. The higher the calibration fidelity, the higher is the operator's confidence in the preview and predictive 
power of the graphics. Through high-fidelity calibration techniques, the graphics images of manipulator and task environment can truly become "virtual realities" to the operator.

Graphics displays of abstractions in teleoperator control are essentially visualizations of invisible events or situations. Such events or situations are: forces exerted by manipulator on objects and on environment; workspace constraints or "holes" in a workspace not accessible under certain task conditions; etc. They provide guidance to the operator. Their performance essentially depends on their formats and on the easy mapping of the display format to the task environment. Here, pointed simplicity plays a key role. The development of such displays is in an embryonic state.

In many cases, human-machine interface devices and techniques in teleoperation are evaluated as separate entities or "subsystems," and their full-valued performance is predicted from such isolated evaluations. Such evaluation methods are useful when different interface devices and techniques are compared. However, a specific device or technique best reveals its virtues or weaknesses when evaluated in a full system operation setting.

In many cases, the "naive user" is used as a "reference operator" for judging or evaluating a human-machine interface device or technique in teleoperator control. Though this idea has a wellmotivated intention, care should be taken to develop a more tangible definition of the "naive user" as a "reference operator." Probably what we mean by a "naive user" when we judge and evaluate a human-machine interface device or technique is: the "natural appeal" of the technique, the easy-toremember features of the device, the amount of learning and training needed to be fully familiar with the device and technique, and so on. The more advanced human-machine interfaces do indeed require familiarization and training before their actual use. In particular, when teleoperation becomes more and more computer aided, the control station will acquire a more and more "abstract" ambience which will have to be mapped to the mechanical world.

The case of multiple operators operating a teleoperator system received little attention from the viewpoint of performance measurements and evaluation methods, though it is a fact that many teleoperator systems are operated by at least two operators. This is particularly true for dual-arm teleoperator systems. The obvious questions are: workload and function distribution, (pilot-copilot; drivernavigator; etc.), and the distribution of responsibilities. A good evaluation method for multiple operator teleoperated systems could also pave the way for defining ways to convert such systems into single-operator systems.

Beth Marcus discussed the notion that evaluations must be task and performance oriented rather than simply based on an abstract notion of "telepresence" or fidelity to the real world.

\section{Comments on Evaluation (Beth Marcus)}

The bottom line from the panel discussion is that evaluation of human interfaces is not easy. The suitability of a given interface for teleoperators or virtual environments is often very task specific. However, it is possible to begin to collect a "wish list" for these devices. All other things being equal the accuracy of sensing should be maximized, training time minimized, and task performance maximized. In addition, the trade-off between performance (i.e., speed, accuracy) and training time should be considered. 
It is important to note that, since no interface hardware today or in the foreseeable future provides all types of feedback and gives a feeling identical to "being there," we must throw away the notion that "natural" equals good. In a non-ideal world, one might be able to convey more information in a more useful form using an artificial presentation. For example, human tactile sense gives no information about proximity, though it may be easy and useful to convey this information through a tactile display.

In order to rapidly expand the utility of virtual environments, we need to take some of the technology that exists in laboratories today and commercialize it to solve real-world problems. Thus, by providing low cost interfaces which advance beyond the capabilities of a mouse or a keyboard, we can have 10,000 or 10 million people contributing to the knowledge base rather than a few hundred. 


\title{
PANEL DISCUSSION ON DESIGN PRINCIPLES AND PREDICTIVE MODELS
}

\author{
H. L. Alexander, Chair (MIT), N. Hogan (MIT), J. M. Hollerback (McGill University), \\ I. Hunter (McGill University), and W. Verplank (I. D. TWO)
}

Questions Discussed:

1. When should master compliance be stiff and when soft? When should slave compliance be stiff and when soft? What are the appropriate places for compliance? When should dampling be added to master or slave?

2. When is it necessary to have an intelligent-computer-mediated telerobot, and when will direct teleoperation suffice? What functions are appropriate to the computer mediator?

3. What machine sensory capabilities are important, in order to support increased computer participation in manipulation? Conversely, what remote human sensory modes are important, and how best to present sensory data to humans?

4. Do current robot designs tend to be too anthropomorphic, and how should we work to design the task (and environment) for the sake of the robot as opposed to designing the robot?

The seminar opened with comments by the panel participants. Bill Verplank introduced the use of metaphors to help drive the design process. As an example, he discussed the relatively familiar desktop metaphor for the computer interface, introduced at Xerox PARC, that has suggestd many aspects of modern interfaces, such as the organization of files (documents) into sets (folders) that can be handled, copied, moved, and displayed analogously to file folders in and on a physical desk.

Bill Verplank introduced a further metaphor, motivated by the computer's functions that may be shared with clothing, as opposed to the desk. He noted that the functions of clothing include physical protection, providing a sense of belonging or membership (as in the case of uniforms or school colors), and generating a sense of satisfaction in ones appearance. The point, again, is to motivate thinking into how a computer might serve the same functions as clothing, and perhaps in ways similar to clothing.

Neville Hogan addressed the adaptation of unfamiliar remote vehicle dynamics, such as the gravity-free dynamics of a teleoperated space vehicle or the viscosity-dominated dynamics of underwater vehicle, in order to present the operator with more natural dynamics to which he/she is accustomed. The point is to adapt the dynamics of the remote environment, through computation and control, to the operator, rather than compelling the operator to adapt his/her self to the remoteenvironment dynamics. 
Neville also addressed the idea of turning manipulator dynamics to tasks, pointing out that humans actually do this relatively seldom, and generally by a modification of posture (for example, in increasing stiffness by strengthening the arm). Antal Bejczy spoke up to defend the use of selective compliance in the performance of manipulation tasks.

John Hollerbach spoke on the design of manipulators, both macro- and micro-, stressing the advantages of parallel-link manipulators in terms of their motion-error behavior and their stiffness. He pointed out that in a parallel-link manipulator, individual errors tend to average rather than to add as in the case of serial-link manipulators, leading to an overall decrease in positioning errors.

Tom Sheridan responded to this, mentioning the differing characteristics of the Jacobian matrices corresponding to parallel- and serial-link manipulators. He went on to introduce a new topic, and a new term, having to do with the design of human-machine interface, for virtual and teleoperator interfaces. He coined the term "haptic teleproprioceptive psychophysics" to refer to the detailed study of human perception of manipulation cues that result from contact with objects. He pointed out that it is important to understand the details of human proprioceptive responses, so that we can take full advantage of that understanding in delivering those cues to the operator, as though force-reflecting hand controllers. * He pointed out that input from persons studying that field, and their participation in meetings such as this one, might greatly benefit teleoperation and virtual environment studies.

Ruzena Bajcsy emphasized the need for much further sensor development, to allow for the detection in the remote environment, of tactile information for reflection to the operator. This led to a discussion of sensor and actuator design, with particular reference to micromachining and its potential in the development of tactile sensors and displays. The group discussed micromachining and its potential for creating actuators able to exert the forces and displacements necessary to excite human touch sensors. Handayam SriniVasan's work in tactile perception sensitivity (which shows that human touch is indeed very sensitive) indicates that such actuators may not be too far out of reach.

This discussion, focusing as it did on the precision of feedback to individual fingers from a dextrous remote manipulator, led to discussion of alternatives, particularly of specialized remote tools to be used in place of general purpose remote manipulators. Nat Durlach prompted lively discussion by inviting designs of a manipulator that improve on the human hand. Two such were mentioned canoe carvers with no pinkie fingers to get in the way, and pianists who have cross connections in their digital tendons severed in order to improve the independent mobility of their fingers. Unfortunately, neither of these manipulators, however improved over the originals, lend themselves to robotic applications at the current time.

Such consideration, however, led to the discussion of the use of muscle fibers as robotic drive elements. This, in turn, introduced discussion of the phase-transition (memory) characteristics of Nitinol wire in the development of actuators displaying high stiffness and force capabilities. It was also suggested that skin grown in vitro might be used for tactile sensing with afferent nerves appropriately connected to electronic sensors and the resulting nerve impulses processed by neural networks.

With regard to specialized tools replacing general-purpose dextrous end-effectors, the point was made that individual tools may be developed that are much better adapted to specific manipulation 
and other tasks, then are currently feasible dextrous manipulators. A general-purpose tool interface, rather than a dextrous end-effector, would serve to attach each such tool to the manipulator wrist and to conduct the appropriate interface services and signals. One such swappable end-effector would of course be a semi-dextrous hand, ranked next to the drill, grinder, wrench, saw, and nut driver. The chairman proposed developing a general-purpose operator "tele-handle" for a teleoperator master arm, that would provide a general-purpose interface to remote or virtual tool, and could supply a wealth of tactile and other feedback to the operator.

Teleoperator tactile feedback having been well-discussed, the subject of tactility in virtual worlds was addressed. Whereas in teleoperator interfaces to remote, physical worlds the problem includes both sensing (remotely) and generating (locally) tactile stimuli, in virtual worlds the sensing problem is eliminated. The problem becomes instead one of synthesizing tactile stimuli, corresponding either literally (as in the case of contact with a hand, unyielding virtual wall) or metaphorically (what does it feel like to have ones hand pass through a virtual wall?) to the virtual reality.

*Chairman's note: With our increased understanding of and capability for tactile feedback, we may want to generalize the term "force-reflecting" to sense-reflecting hand-controllers. An example is Steve Jacobson's controller which delivers the tap responses to the operator on contact with objects, which response is not merely derived from force feedback, but is deliberately synthesized to represent to the operator the contact event. 
MINI SEMINAR: TELEOPERATORS WITH MICRO SLAVE ROBOTS

K. J. Gabriel, Chair

University of Tokyo 
Q 2119

\section{POSTER PRESENTATIONS}





\title{
THE HUMAN OPERATOR TRANSFER FUNCTION: IDENTIFICATION OF THE LIMB MECHANICS SUBSYSTEM
}

\author{
Lynette A. Jones and Ian W. Hunter \\ McGill University \\ Montreal, Quebec, CANADA
}

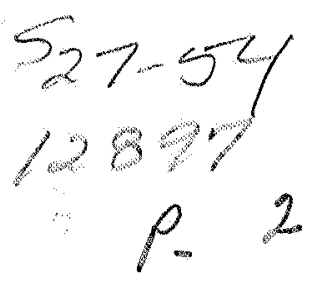

In most studies of human manual control systems, the operator is characterized as a lumped, indivisible system, whose dynamics are linear and of low order. The objective of our research is to decompose the performance of the human operator in terms of the subsystems that determine the operator's responses in order to establish how the dynamics of these component subsystems influence the operator's performance. In the present experiment, the dynamic stiffness of the human elbow joint was measured at rest and under different levels of biceps muscle activation; this work forms part of the analysis of the limb mechanics subsystem.

The stiffness of the elbow joint was determined by subjecting the right wrist to stochastic position perturbations (S.D. $3 \mathrm{~mm}$, bandwidth of $100 \mathrm{~Hz}$ ) delivered by an electromagnetic linear motor (100 mm maximum linear displacement, peak static force $500 \mathrm{~N}$ ). Subjects generated a target force (ranging from 0 to $150 \mathrm{~N}$ ) during the perturbations, and were provided with visual feedback of the mean force produced.

The stiffness frequency response function was determined at each mean force level. These functions were calculated by Fourier transforming the stiffness impulse response functions. Figure 1 shows the stiffness frequency response functions obtained from a subject generating a mean force of $25 \mathrm{~N}$ (thin line) and 150N (thick line). The increase in the low frequency stiffness with an increase in mean muscle activation level (as reflected in the mean force) is clearly evident. The figure also demonstrates that stiffness increases at higher frequencies. This essentially high-pass characteristic of the limb mechanics system is well-known. In Figure 2, a typical coherence function is shown. The decrease in coherence at higher frequencies is due, in part, to a loss in the signal-to-noise ratio caused by limitations in the bandwidth of the motors, whereas the loss of coherence at lower frequencies probably reflects the contribution of reflexes. 
Figure 1

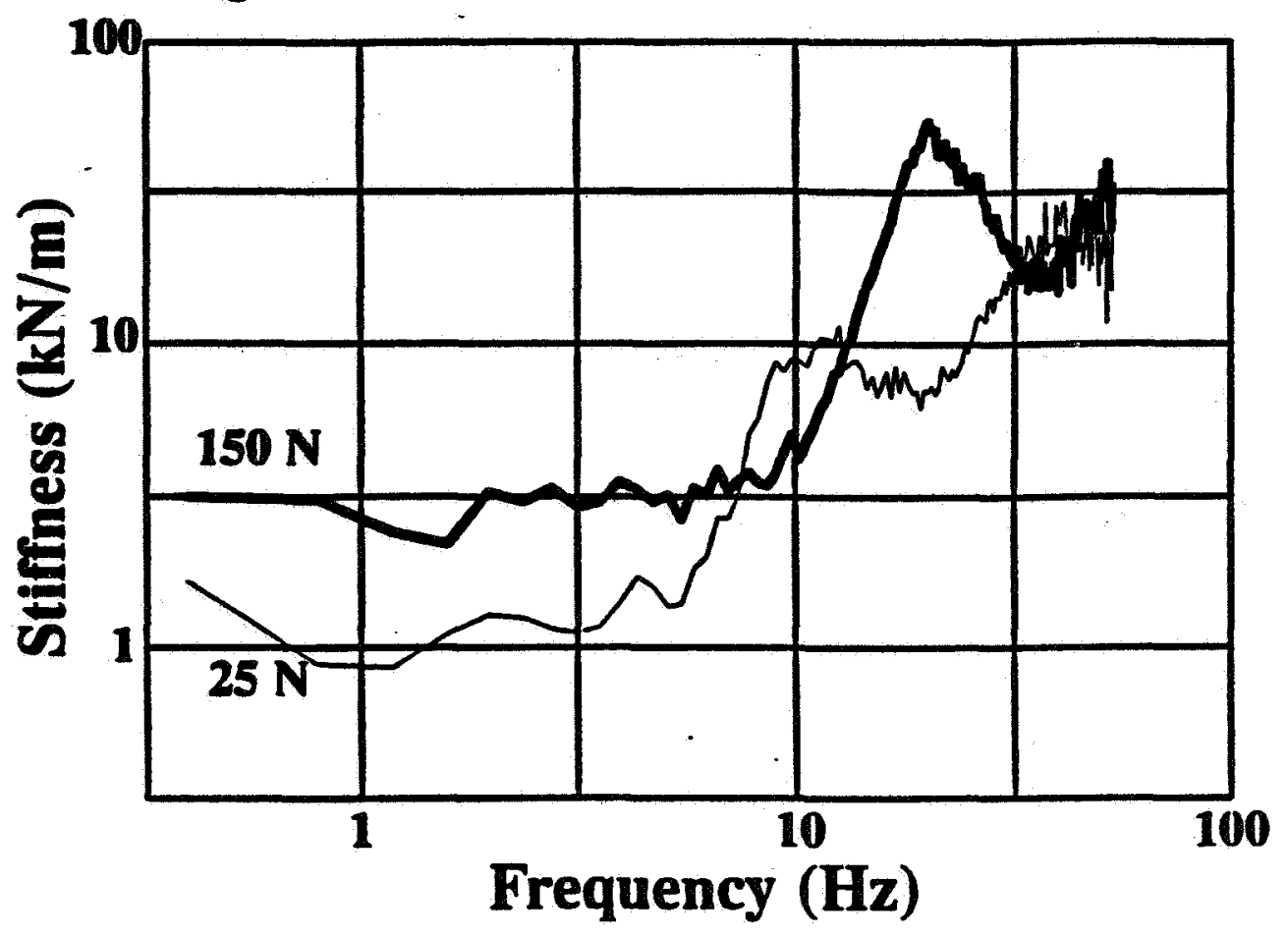

Figure 2

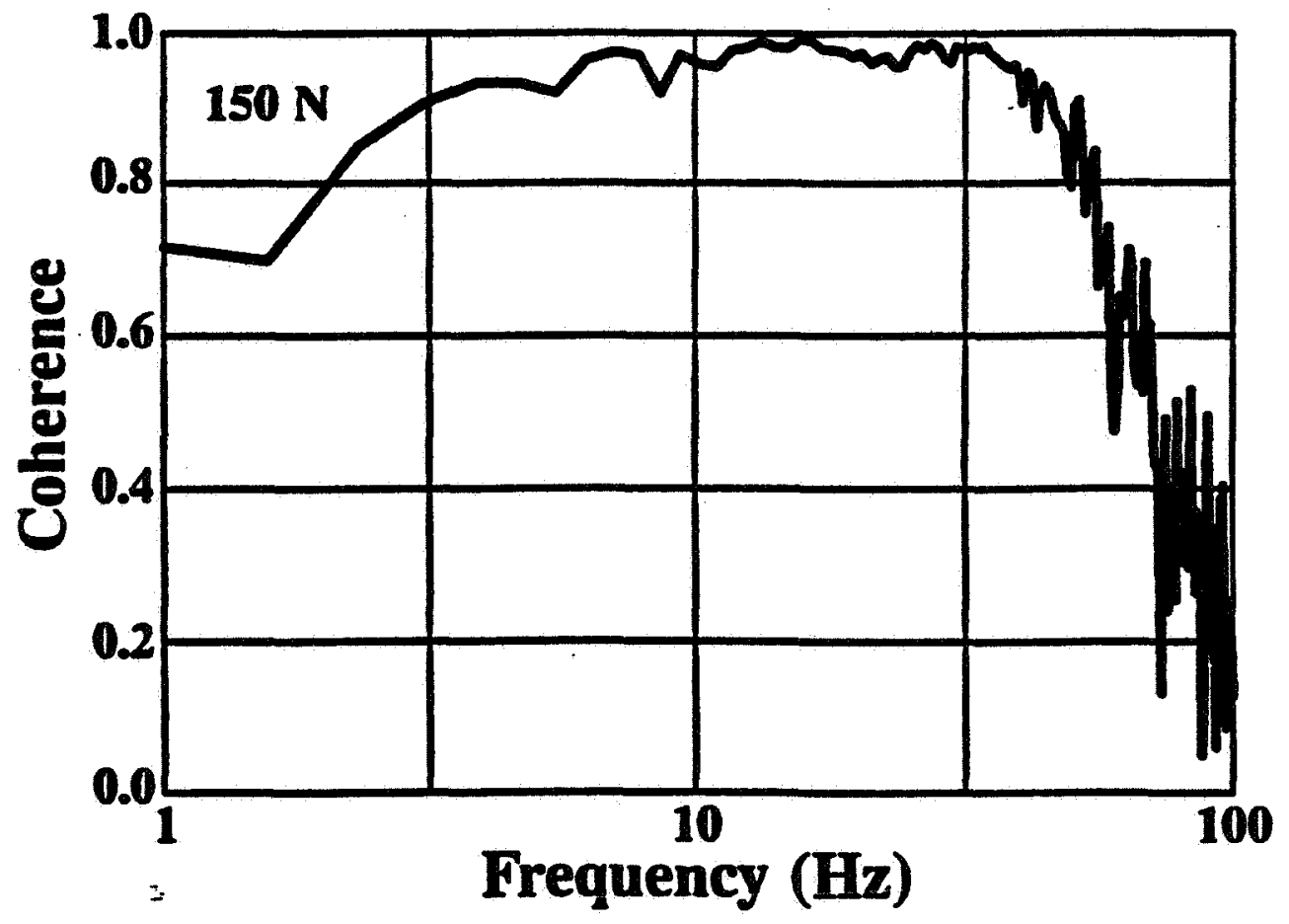




\title{
TACTILE DISCRIMINATION AND REPRESENTATIONS OF TEXTURE, SHAPE, AND SOFTNESS
}

\author{
M. A. Srinivasan \\ Research Lab of Electronics \\ Massachusetts Institute of Technology \\ Cambridge, Massachusetts \\ and \\ R. H. LaMotte \\ Department of Anesthesiology \\ Yale University School of Medicine \\ New Haven, Connecticut
}

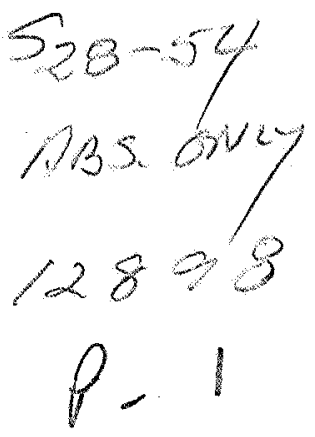

We present here some of the salient results on the tactual discriminabilities of human subjects obtained through psychophysical experiments, and the associated peripheral neural codes obtained through electrophysiological recordings from monkey single nerve fibers. Humans can detect the presence of a 2 microns high single dot on a smooth glass plate stroked on the skin, based on the responses of Meissner type rapidly adapting fibers (RAs). They can also detect a 0.06 microns high grating on the plate, owing to the response of Pacinian corpuscle fibers. Among all the possible representations of the shapes of objects, the surface curvature distribution seems to be the most relevant for tactile sensing. Slowly adapting fibers respond to both the change and rate of change of curvature of the skin surface at the most sensitive spot in their receptive fields, whereas RAs respond only to the rate of change of curvature. Human discriminability of compliance of objects depends on whether the object has a deformable or rigid surface. When the surface is deformable, the spatial pressure distribution within the contact region is dependent on object compliance, and hence information from cutaneous mechanoreceptors is sufficient for discrimination of subtle differences in compliance. When the surface is rigid, kinesthetic information is necessary for discrimination, and the discriminability is much poorer than that for objects with deformable surfaces. 

INTERFACE

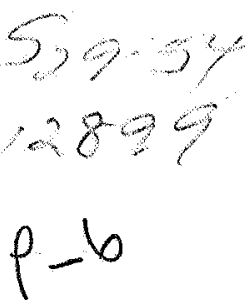

\author{
Bernard D. Adelstein and Michael J. Rosen \\ Massachusetts Institute of Technology \\ Cambridge, Massachusetts
}

\begin{abstract}
This summary focuses on the kinesthetic interface of a virtual invironment system that was developed at the Newman Laboratory for Biomechanics and Human Rehabilitation at M.I.T. for the study of manual control in both motorically impaired and able-bodied individuals.
\end{abstract}

\title{
MECHANISM DESIGN
}

The kinesthetic interface is an electromechanically actuated manipulandum (i.e., backdriveable manipulator) that enables dynamic interaction between the arm of a human operator/experimental subject and controlled mechanical loads. The manipulandum, depicted in Figure 1, consists of a direct-drive handle linkage which is coupled to two printed armature brushed DC motors. The interface is instrumented to sense displacements, velocities, and accelerations at the motor shafts, and forces between the hand grip and the linkage.

The coupling mechanism that joins the handle to the motors forms a spherical closed-chain fivebar linkage, resulting in two kinematic degrees of freedom at the hand grip. This linkage permits both actuator housings to be anchored to a common mechanicsl ground, thereby making it unnecessary to carry their excessive weight and inertia. Because the linkage consists of only ball bearing revolute pairs and rigid links, undesirable friction, backlash, and compliance characteristics associated with other transmission types are greatly reduced in this interface.

Additional attributes of this mechanism configuration prove advantageous for manipulandum control. Over its $17 \mathrm{~cm}$ square workspace, the interface is approximately planar, and, most importantly, the actions of the two motors are nearly decoupled.

\section{SYSTEM CONTROL}

The overall virtual environment system, shown in Figure 2, consists of the kinesthetic interface and a planar video display. System operation is supervised by an LSI-11/23 based micro computer, responsible for collecting and storing data, commanding and monitoring the Control Interface Unit (CIU), and transferring target and tracking response information to the Amiga 1000 personal 
computer for display to the subject. The CIU contains hardwired circuitry to control load simulations by each motor as well as maintain system safety.

Because of the manipulandum's nearly planar and nearly decoupled linkage kinematics, configuration space control can be used to regulate endpoint impedance directly, without geometric computation. As a consequence, the LSI-11/23 host can update CIU load control parameters at rates exceeding $1 \mathrm{kHz}$.

\section{MECHANICAL LOAD AND OBJECT SIMULATION}

The manipulandum's ability to produce positive (i.e., resistive) and negative (i.e., destabilizing) spring and damping fields was examined. Load ranges achieved with the CIU's hardwired digitallysupervised analog controller are listed in Table 1. Greater stiffness-up to 3 times higher-are obtainable under purely digital control implementations.

\begin{tabular}{|l|c|c|}
\hline Load Type & Rotational & Translational Equivalent \\
\hline Active Resistive Stiffness & $0-136 \mathrm{~N}-\mathrm{m} / \mathrm{rad}$ & $0-3100 \mathrm{~N} / \mathrm{m}$ \\
Active Resistive Damping & $0-6.7 \mathrm{~N}-\mathrm{m} / \mathrm{rad} / \mathrm{s}$ & $0-152 \mathrm{~N} / \mathrm{m} / \mathrm{s}$ \\
Active Inertia & $.007-0.045 \mathrm{~kg}-\mathrm{m}^{2}$ & $.13-1 \mathrm{~kg}$ \\
Maximum Torque/Force & $4.7 \mathrm{~N}-\mathrm{m}$ & $21 \mathrm{~N}$ \\
\hline
\end{tabular}

Table 1: Load ranges achieved under digitally supervised analog control. The translational load equivalent is calculated for the hand grip end of the fixed length handle.

In addition to simulating mechanical impedances, the controller can either mask or enhance the manipulandum's passive inertia and friction, thereby allowing the handle to feel "invisible" when pushed and have sufficient friction to stay in place when "let go" by the human operator. Contact with a hard non-sticky wall anc capture by a detent also have been simulated convincingly by the manipulandum, as illustrated in Figures 3 and 4. Together, the performance capabilities described in this paragraph successfully meet a set of criteria used in the industry to demonstrate that an "artificial control feel" device is versatile enough to faithfully execute any general simulation (Jex 1988).

\section{REFERENCE}

H. R. Jex. Four critical test for control-feel simulators. Proceedings of the 23rd Conference on Manual Control, Cambridge MA, June 1988. 


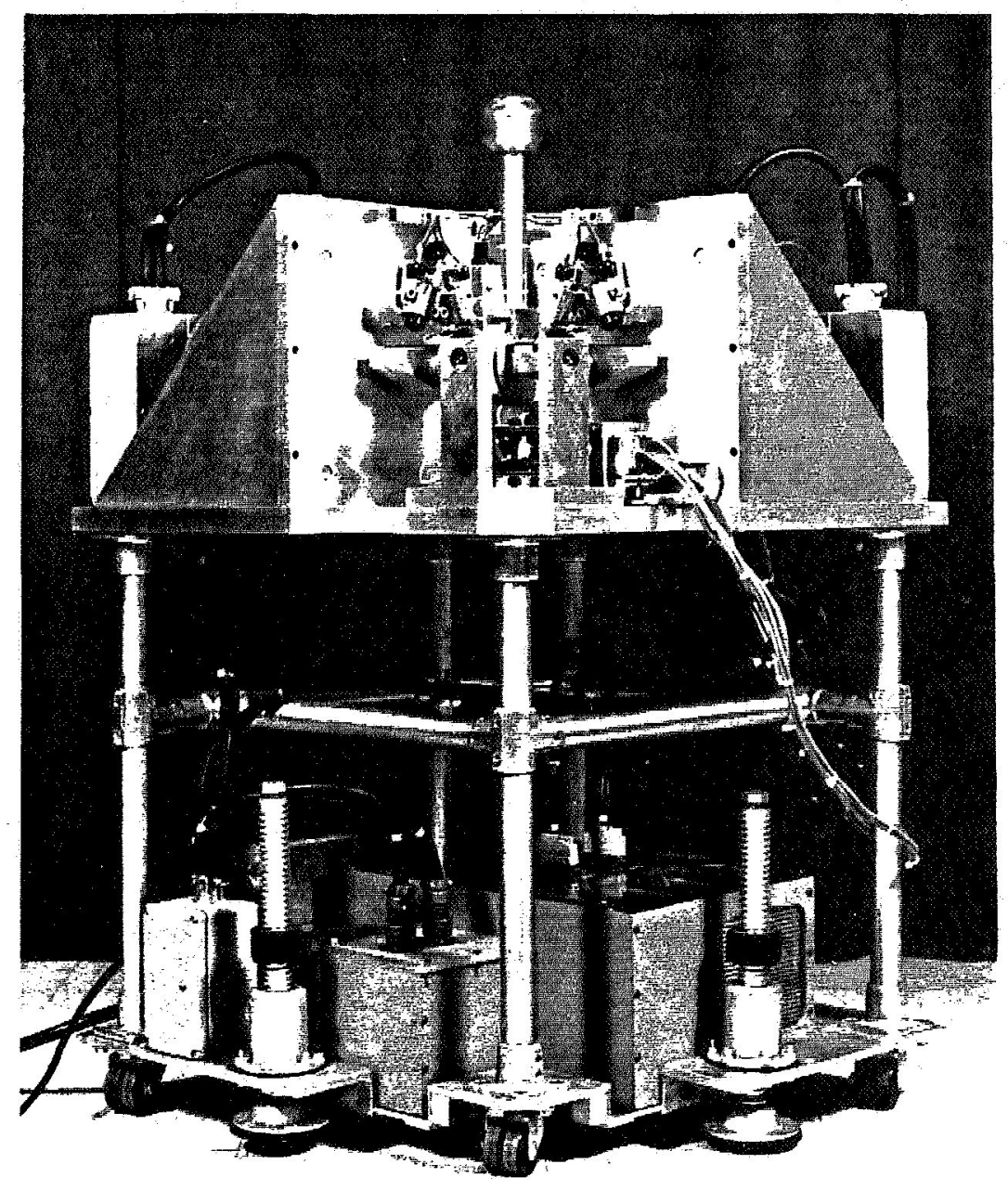

Figure 1. The manipulandum. Height from floor to spherical hand grip is 1 meter. 


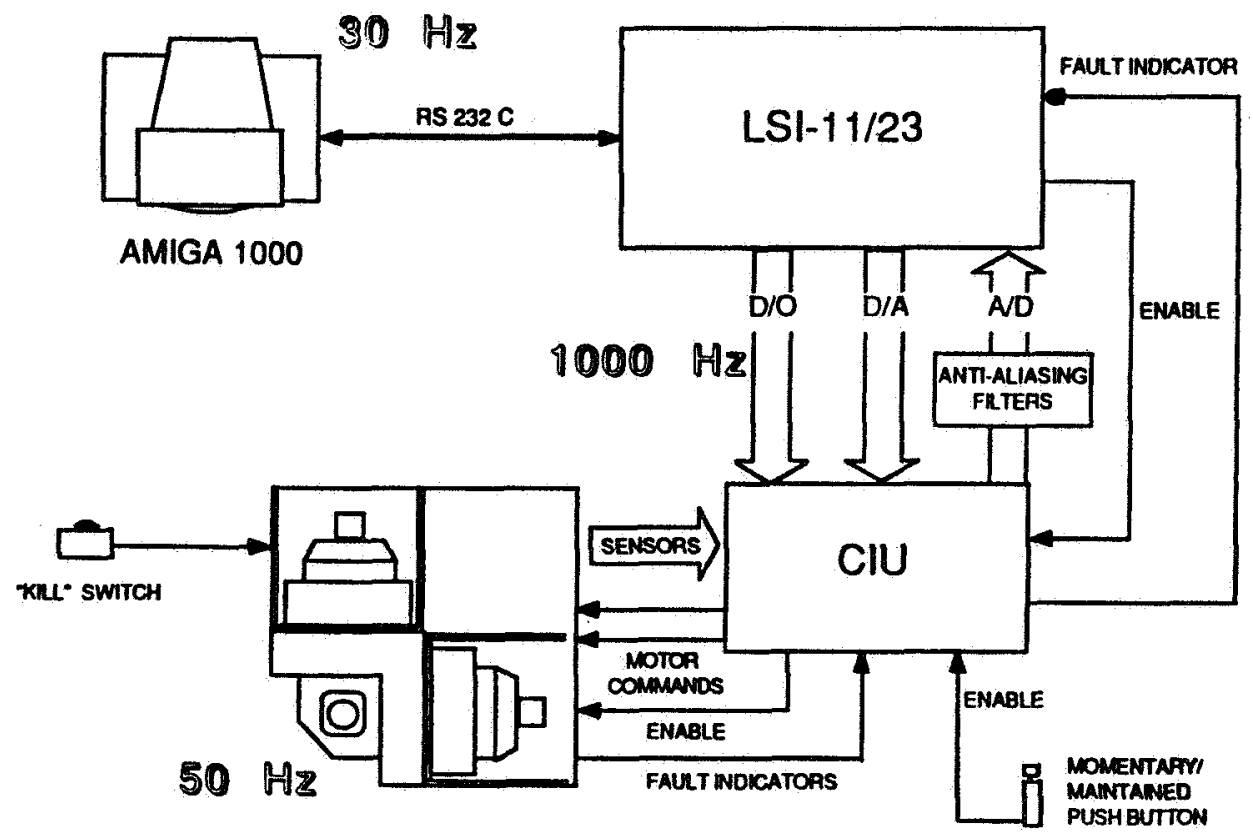

Figure 2. Virtual environment system components and communication. The highlighted frequencies (clockwise from upper left ). indicate the computer update rates for the visual display and load controller, and the manipulandum force bandwidth as determined by its first structural mode. 

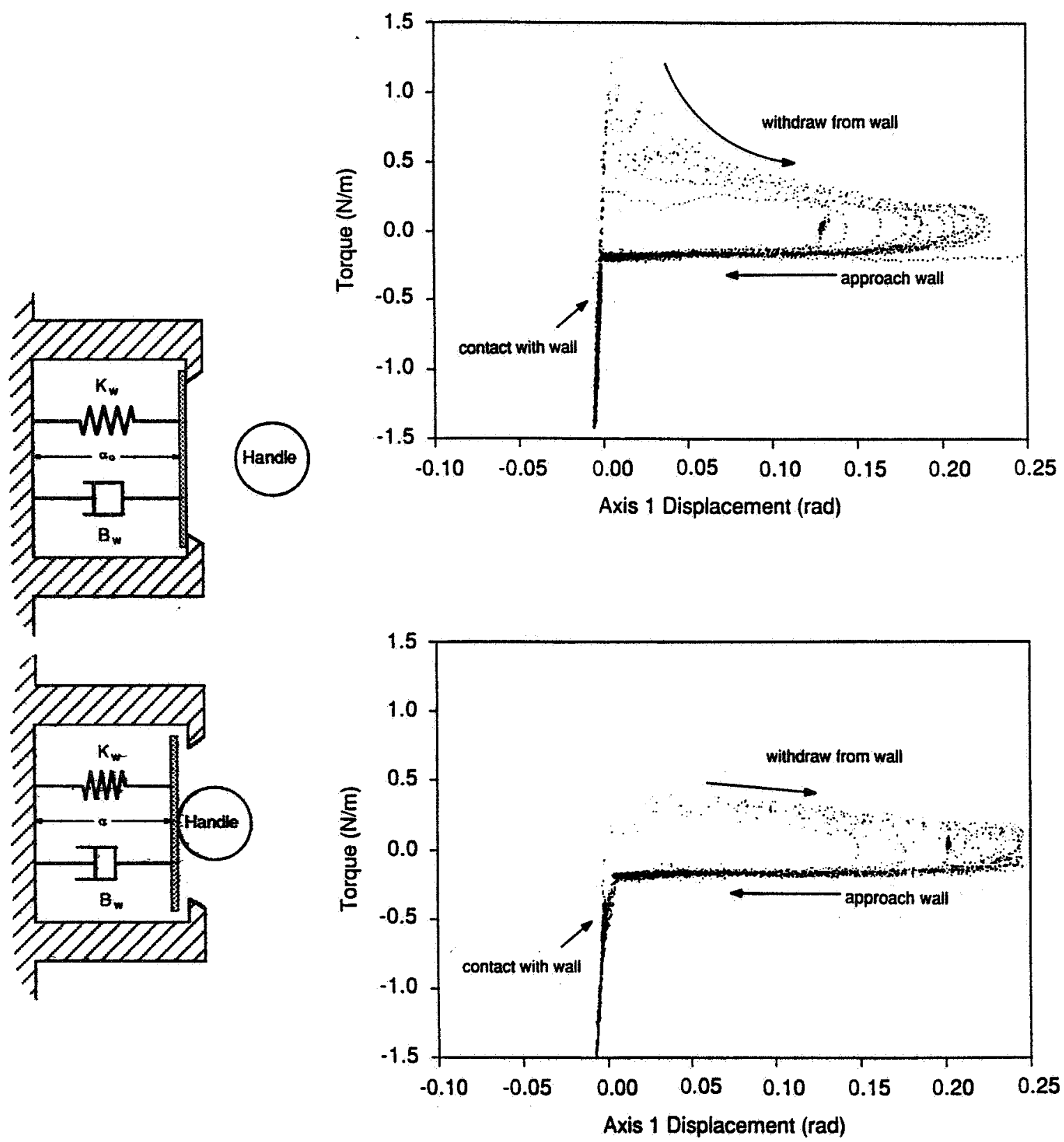

Figure 3. Wall model and implementations. The wall is modelled (left $)$ as a massless plate backed by a stiff spring and viscous damper. When the handle is to the right of the wall rest position (top ), no resistance is encountered. As the handle moves past the wall rest position (bottom), the spring and dampers oppose further motion to the left. Torques resulting from the hand force applied to the constant length manipulandum handle are plotted against displacement (right) to demonstrate the effect of bandwidth on wall quality. The upper plot shows wall performance with a $20 \mathrm{msec}$ lag introduced prior to detection of the handle position by the computer. The lower plot has this lag removed. Both simulations are updated at $1 \mathrm{kHz}$. The lag causes a perceived "stickiness," visible in the upper plot as the attractive force pulling the handle back toward the wall (i.e., displacement $=0$ ). Away from the wall, the difference between mean force levels during approach and withdrawal motions is due to uncompensated friction. 

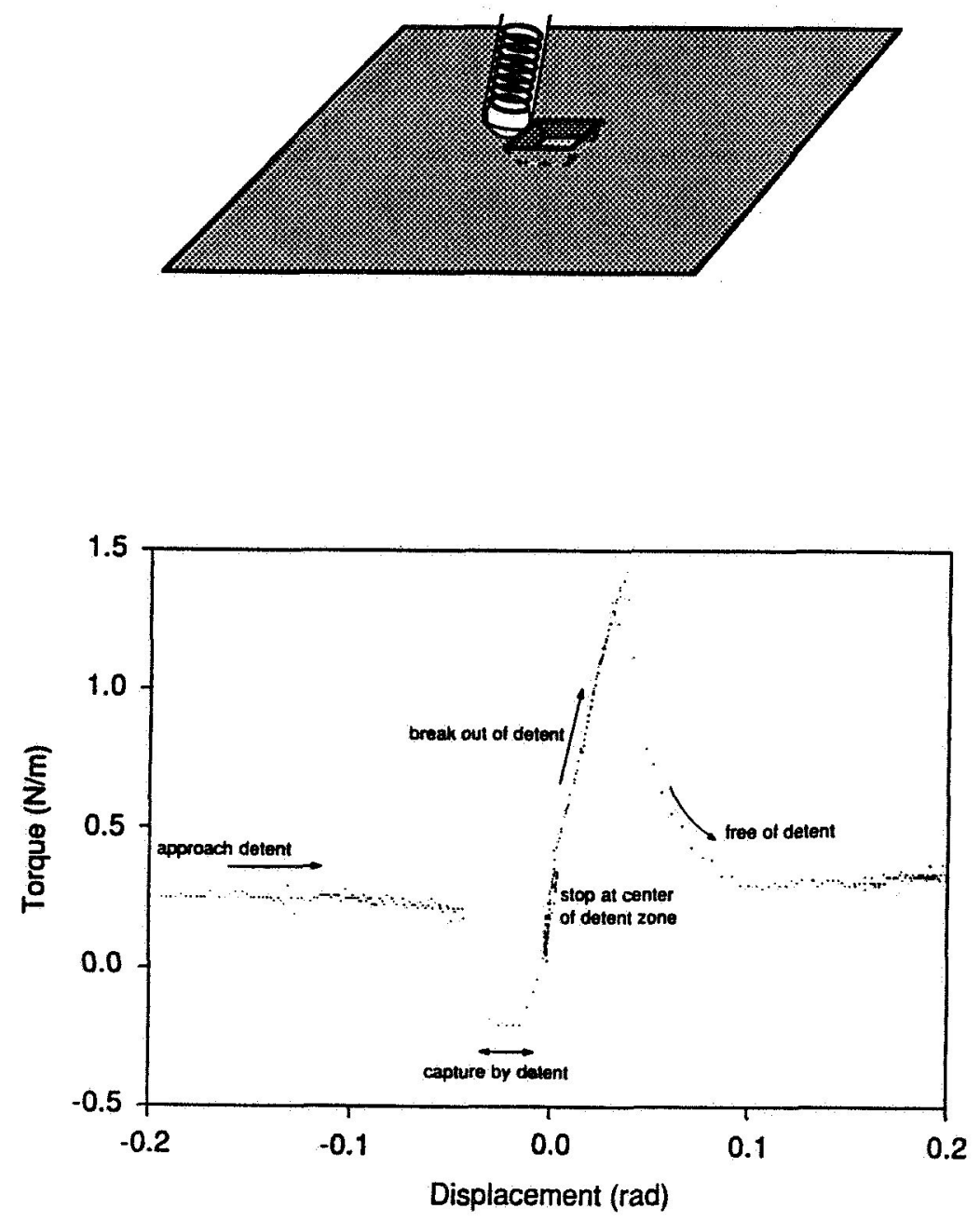

Figure 4. Detent model and implementation. The detent is modelled (top) as a spring loaded ball plunger located at the bottom of the manipulandum handle that is trapped when it passes over the indentation in the planar workspace. The plot shows the history of torque due to handle force versus handle displacement in one of the manipulandum degrees of freedom as the handle is captured by a simulated detent, comes to a stop, and then breaks free. 


\title{
PERCEPTUAL AND PERFORMANCE CONSEQUENCES OF FLIGHT IN VIRTUAL WORLDS
}

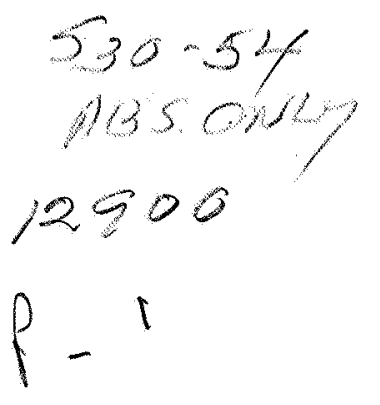

\author{
C. Thomas Bennett and Martin Schwirzke \\ NASA Ames Research Center \\ Moffett Field, California \\ and \\ James S. Tittle \\ University of California \\ Irvine, California
}

Head-mounted, virtual world technologies were developed in the 1960s. Comeau and Bryan (1961) reported on a head-slaved video control system. In 1968, Sutherland discussed a head-slaved, graphics based virtual world that was later demonstrated by Vickers (1970). Operational systems for military aircraft were quickly developed and tested (Johnson and Foster, 1977).

As part of second generation technology efforts, new display media and computer graphics systems have been developed (Fisher, McGreevey, Humphries, and Robinett, 1986). With this new technology effort has come more systematic studies of the performance consequences resulting from conducting teleoperations in aeronautical settings.

There are two primary purposes for head-mounted systems in aeronautical settings. One is for helmet-mounted sights and teleoperated (head-slaved) weapons systems. Bennett, Johnson, Perrone, and Phatak (1988) evaluated head tracking performance during passive and controlled flight. In that study, comparisons were also made of head tracking performance in sterile and relatively complex virtual worlds. That study confirmed the robustness of head tracking performance across a wide variety of visual scenes.

A second use of virtual world displays is for aircraft control. Aircraft controllability using headmounted, panel-mounted or simulated out-the-window scenes has been systematically examined (Bennett, O'Donnell, and Johnson, 1988; O'Donnell, Johnson, and Bennett, 1988). Those studies reported the range of rotorcraft flight tasks in which head-mounted virtual worlds provided some advantages.

Two studies will be reported that examine the perceptual/performance effects of virtual worlds. The first examines head tracking performance with roll-stabilized versus non-roll stabilized virtual worlds. The purpose of the study was to (a) examine display strategies used in current display systems and (b) study the adaptability of observers to estimated glide slope angles using head-slaved versus head-stabilized imagery. The purpose of this study was to examine the usefulness of wide field-of-regards during final approaches to a runway. 


\section{A DECADE OF TELEROBOTICS IN REHABILITATION: DEMONSTRATED UTILITY BLOCKED BY THE HIGH COST OF MANIPULATION AND THE COMPLEXITY OF THE USER INTERFACE}

Larry Leifer, Stefan Michalowski, and Machiel Van Der Loos

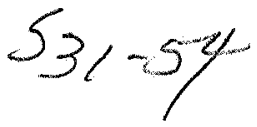

Stanford University

Stanford, California

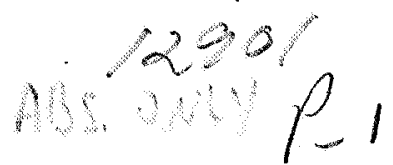

The Stanford/VA Interactive Robotics Laboratory set out in 1978 to test the hypothesis that industrial robotics technology could be applied to serve the manipulation needs of severely impaired individuals. Five generations of hardware, three generations of system software, and over 125 experimental subjects later, we believe that genuine utility is achievable. The experience includes development of over 65 task applications using voiced command, joystick control, natural language command and 3D object designation technology.

A brief foray into virtual environments, using flight simulator technology, was instructive. If reality and virtuality come for comparable prices, you cannot beat reality.

A detailed review of assistive robot anatomy and the performance specifications needed to achieve cost/beneficial utility will be used to support discussion of the future of rehabilitation telerobotics. Poised on the threshold of commercial viability, but constrained by the high cost of technically adequate manipulators, this worthy application domain flounders temporarily. In the long run, it will be the user interface that governs utility. 


\title{
A PHYSICAL CONTROL INTERFACE WITH PROPRIOCEPTIVE FEEDBACK AND MULTIPLE DEGREES OF FREEDOM
}

\author{
G. H. Creasey, D. Gow, Y. Sloan, and B. Meadows \\ University of Edinburgh and Princess Margaret Rose Orthopaedic Hospital \\ Edinburgh, SCOTLAND
}

The use of the drug thalidomide by pregnant mothers in Britain resulted in a variety of deformities including the birth of children having no arms. Such children were provided with powered artificial arms with up to five degrees of freedom simultaneously controlled in real time by shoulder movement (ref. 1) and whose operation could be learnt by children within a matter of hours. The ease with which this manipulation could be learnt and used may have been due to the system used to provide feedback of position and force to the user's skin and joints. In this way, the physiological sense of proprioception was extended from the user into the device, reducing the need for visual feedback and conscious control.

With the banning of thalidomide, this technique fell into disuse but it is now being re-examined as a control mechanism for other artificial limbs (refs. 1-5) and it may have other medical applications to allow patients to control formerly paralysed limbs moved by electrical stimulation (ref. 6). It may also have commercial applications in robotic manipulation or physical interaction with virtual environments.

To allow it to be investigated further, the original pneumatic control system has recently been converted to an electrical analogue to allow interfacing to electronic and computer-assisted systems. A harness incorporates force-sensitive resistors and linear potentiomenters for sensing position and force at the interface with the skin, and miniature electric motors and lead screws for feeding back to the user the position of the robotic arm and the forces applied to it. In the present system, control is applied to four degrees of freedom using elevation/depression and protraction/retraction of each shoulder so that each collar-bone emulates a joystick. However, both electrical and mechanical components have been built in modular form to allow rapid replication and testing of a variety of force and position control strategies.

\section{REFERENCES}

1. Simpson DC. The development and the control of a powered prosthesis for children. Health Bulletin (1964) 22(4):67-69

2. Gow DJ, Dick T, Draper E, Loudon I \& Smith P. Physiologically appropriate control of an electrically powered hand prosthesis. Proc 4th World Congress of Int Soc of Prosthetics \& Orthotics, London, 1983, p.41 
3. Doubler JA \& Childress DS. An analysis of extended physiological proprioception as a prosthesis-control technique. J Rehab Research and Devt (1984) 21(1):5-18

4. Doubler JA \& Childress DS. Design and evaluation of a prosthesis control system based on the concept of extended physiological proprioception. J Rehab Research and Devt (1984) 21(1):19-31

5. Kirtley C. Extended physiological proprioception. In Neural Protheses: Motor System, Engineering Foundation, New York, 1988, p.85

6. Johnson MW \& Peckham PH. Evaluation of shoulder position as a command control source for use with neural prosthetic devices by quadriplegic individuals. Proc Ann Conf Rehab Eng Soc North America (1986) pp.454-456 


\section{"ROTATIONAL WIND" INDICATOR ENHANCES CONTROL OF ROTATED DISPLAYS: H. A. CUNNINGHAM AND M. PAVEL}

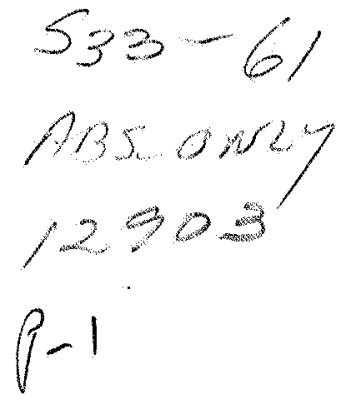

\author{
H. A. Cunningham and M. Pavel \\ Stanford University \\ Stanford, California \\ and \\ NASA Ames Research Center \\ Moffett Field, California
}

Rotation by $108^{\circ}$ of the spatial mapping between a visual display and a manual input device produces large spatial errors in a discrete aiming task. These errors are not easily corrected by voluntary mental effort, but the central nervous system does adapt gradually to the new mapping. Bernotat (1970) showed that adding true hand position to a $90^{\circ}$ rotated display improved performance of a compensatory tracking task, but tracking error rose again upon removal of the explicit cue. This suggests that the explicit error signal did not induce changes in the neural mapping, but rather allowed the operator to reduce tracking error using a higher mental strategy.

In this report, we describe an explicit visual display enhancement applied to a $108^{\circ}$-rotated discrete aiming task. A "wind indicator" corresponding to the effect of the mapping rotation is displayed on the operator-controlled cursor. The human operator is instructed to oppose the virtual force represented by the indicator, as one would do if flying an airplane in a crosswind. This enhancement reduces spatial aiming error in the first 10 minutes of practice by an average of $70 \%$ when compared to a no enhancement control condition. Moreover, it produces adaptation aftereffect, which is evidence of learning by neural adaptation rather than by mental strategy. Finally, aiming error does not rise upon removal of the explicit cue.

\section{REFERENCE}

Bernotat, R.K. (1970) Rotation of visual reference systems and its influence on control quality. IEEE Transactions on Man-Machine Systems, vol. MMS-11, 129-131.

This research was supported by Air Force Office of Scientific Research Grant \#84-03-08, by National Aeronautics \& Space Administration Grant \#NCC-2-307, and by National Research Council Associateship funding to author Cunningham. 
OBJECTIVE EVALUATION OF SITUATION AWARENESS FOR DYNAMIC DECISION MAKERS IN TELEOPERATIONS

\author{
Mica R. Endsley \\ Northrop Corporation \\ Lomita, California
}

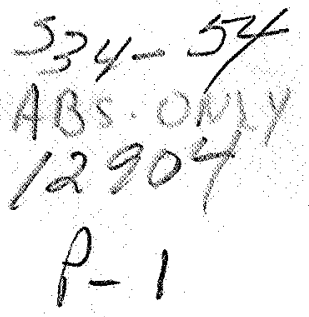

Situation awareness, a current mental mode of the environment, is critical to the ability of operators to perform complex and dynamic tasks. This should be particularly true for teleoperators, who are separated from the situation" they need to be aware of. The design of the man-machine interface must be guided by the goal of maintaining and enhancing situation awareness.

The objective of this work has been to build a foundation upon which research in the area can proceed. A model of dynamic human decision making which is inclusive of situation awareness will be presented, along with a definition of situation awareness. A method for measuring situation awareness will also be presented as a tool for evaluating design concepts. The Situation Awareness Global Assessment Technique (SAGAT) is an objective measure of situation awareness originally developed for the fighter cockpit environment. The results of SAGAT validation efforts will be presented. Implications of this research for teleoperators and other operators of dynamic systems will be discussed. 


\title{
DEVELOPMENT OF VISUAL 3D VIRTUAL ENVIRONMENT FOR CONTROL SOFTWARE
}

\author{
Michitaka Hirose, Takeshi Myoi, Haruo Amari, Kohei Inamura, and Lawrence Stark \\ University of Tokyo and Tokyo Electric Power Co., Tokyo, JAPAN \\ and University of California, Berkeley California
}

Virtual environments for software visualization may enable complex programs to be created and maintained. A typical application might be for control of regional electric power systems. As these encompass broader computer networks than ever, construction of such systems becomes very difficult. Conventional text-oriented environments are useful in programming individual processors. However, they are obviously insufficient to program a large and complicated system, that includes large numbers of computers connected to each other; such programming is called "programming in the large."

As a solution for this problem, the authors are developing a graphic programming environment wherein one can visualize complicated software in virtual 3D world. One of the major features of the environment is the 3D representation of concurrent process. 3D representation is used to supply both network-wide interprocess programming capability (capability for "programming in the large") and real-time programming capability. The authors' idea is to fuse both the block diagram (which is useful to check relationship among large number of processes or processors) and the time chart (which is useful to check precise timing for synchronization) into a single 3D space. The 3D representation gives us a capability for direct and intuitive planning or understanding of complicated relationship. among many concurrent processes.

To realize the 3D representation, a technology to enable easy handling of virtual 3D object is a definite necessity. Using a stereo display system and a gesture input device (VPL DataGlove), our prototype of the virtual workstation has been implemented. The workstation can supply the "sensation" of the virtual 3D space to a programmer. Software for the 3D programming environment is implemented on the workstation. According to preliminary assessments, a 50\% reduction of programming effort is achieved by using the virtual 3D environment. The authors expect that the 3D environment has considerable potential in the field of software engineering.

\section{PROGRAMMING IN THE LARGE}

The authors are involved in the design project for future power supply system in Tokyo. The goal of the project is to have the entire power distributing system controlled automatically by a vast computer networking system. In order to realize that goal, we need to find ways to solve the problems which arise in developing such a large and complicated software scheme.

Two basic types of problems associated with the different levels of the networking environment can be considered. One type of question might be: "How do I program each computer?" (This is called programming in the small). Another mights be: "How do I coordinate many computers?." (This is called programming in the large.) 
Since the programming environment itself is built on "algorithmic" or "logical" characteristics, it is convenient to use text representation. With text, we can eliminate much ambiguity. However, text alone becomes limitingly insufficient in the case of "programming in the large" where the most important factor in the field concerns total configuration design rather than detailed logic design. Thus a more global methodology is needed to correct for faults in the overall system design, even though each individual subsystem may be correctly designed.

In "programming in the large," the authors believe that visual representations play an important role in the intuitive understanding of software and a combination of text and visual environments will provide the best solution.

\section{CONCEPT OF 3D VISUAL REPRESENTATION}

Often, a network diagram is given as a $2 \mathrm{D}$ representation, known as block diagram. However, if the description of a real-time control program is required, such as in the synchronization of several control processes, the time dimension should also be taken into consideration.

Fig. 1 shows the concept of the 3D visual representation. If we observe the representation along the $\mathrm{X}$ axis, it will be a conventional block diagram. The axis normal to the $\mathrm{X}$ axis is a time chart. Using this $3 \mathrm{D}$ representation, the programmer can intuitively grasp the state of concurrent process.

In the $3 \mathrm{D}$ visual representation, the geometrical shape used to represent program flow of successive message passing is also important. The shape itself indicates the type of information exchange. Sometimes the programmer can handle the software entirely based on its shape. The greater the number of processes, the greater the advantages of $3 \mathrm{D}$ representation, presumably due to characteristics of the human cognitive processes.

\section{IMPLEMENTATION}

Several years ago, such an idea was unrealizable due to the limitations of computer capabilities. To implement 3D representations, we need a new technology able to incorporates real-time 3D animation, stereo displays, 3D input devices, and other aspects of the virtual 3D work-space. To generate a 3D image, the authors used Stereographics CrystalEyes System, (a flickerless 3D CRT by high speed LC (120 Hz Liquid Crystal) shutter). By adding a head movement detector (Polhemus 3D tracker) to that system, a partial "look-around" effect can be implemented. As a gestural input device, VPL DataGlove is used.

Fig. 2 shows the current hardware configuration of the virtual 3D workstation. The system includes two sub-workstations. One of them (an HP9000 SRX) is used for generating the virtual 3D image and handling I/O devices. Another ( a Sun 3) is used for text handling. In other words, the first one is for "programming in the large," and the second is for "programming in the small."

Figure 3 shows an example of the visual programming environment. The message flow between processes is displayed in virtual 3D space. One small box represents a computing object (sometimes it can be a process, sometimes it can be a processor). 
Semi-tranparent box represents a carrier of computing objects (it can be a stand-alone computer box).

Using a virtual hand which works as 3D pointer and a kind of "action menu" which detects gestures, programmer can handle virtual objects (such as "objects" and "messages") in the virtual 3D world. Currently, the environment has two modes, called "world mode" and "succession mode." World mode is mainly for observing whole shapes of the software. Succession mode is used for editing messages passing among objects. The configuration is still preliminary. Further investigation is needed concerning the design of object handling in 3D virtual space.

Tools included in the environment are as follows:

1. Virtual Measure/Ruler: Tool to measure the exact relationship among virtual objects.

2. Critical Path Finder: Tool to find and display the critical path which determines the total network throughput for a given network task.

3. Network Simulator: Tool to simulate message passing and data processing on each computer.

4. Network Planner: Tool to update information about the network configuration. This tool works with Network Simulator.

\section{ASSESSMENTS AND CONCLUSION}

As a preliminary assessment of the 3D environment, several multiprocessor programming tasks were assigned to subjects. The target system is supposed to be 3-4 networked personal computers. Completion time with and without the environment was measured. Roughly speaking, completion time with the 3D environment was shorter than that of the conventional methodology. In addition, using $3 \mathrm{D}$ lowers the variance, indicating that the environment reduces error.

The authors believe that the concept of 3D visual representations has proven to be effective even in the simple environment already implemented. A far greater impact can be expected in a more sophisticated environment as the most case of software environment. For example, Smalltalk80 is highly valued for its concept of "object oriented" programming, but its environment is valued much more than the concept itself. The authors consider this kind of practical effort is indispensable. From a practical point of view, however, much effort is needed to prepare and accumulate effective tools for this new environment. 


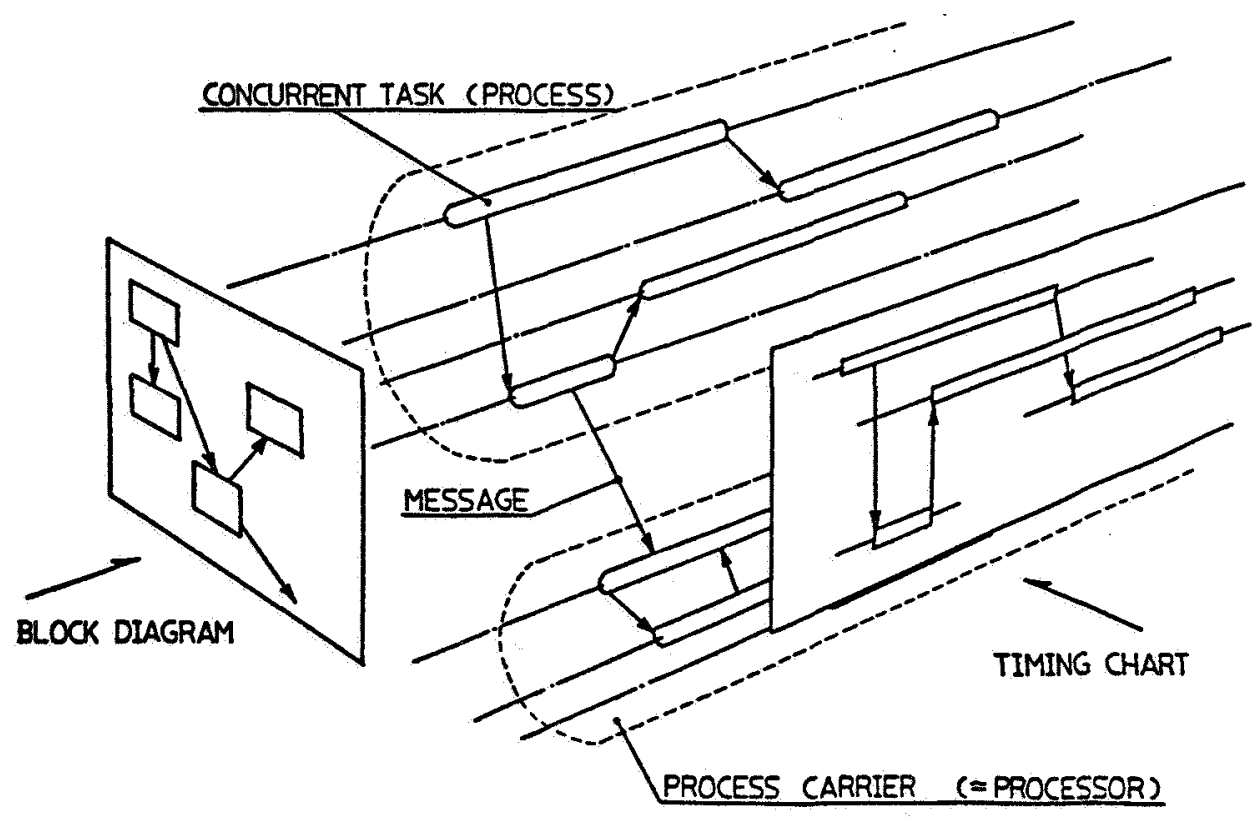

Figure 1. Concept of 3D visual representation.

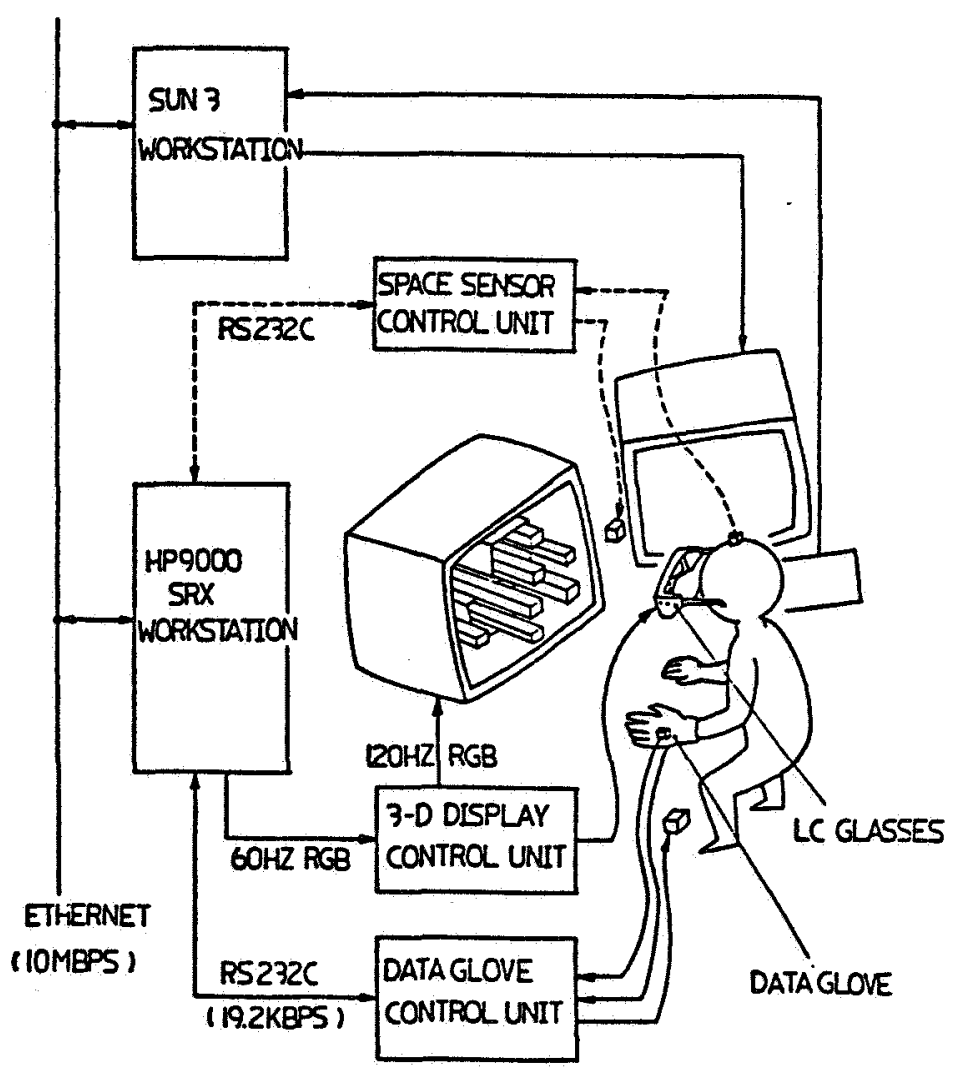

Figure 2. Hardware configuration of the virtual 3D workstation. 


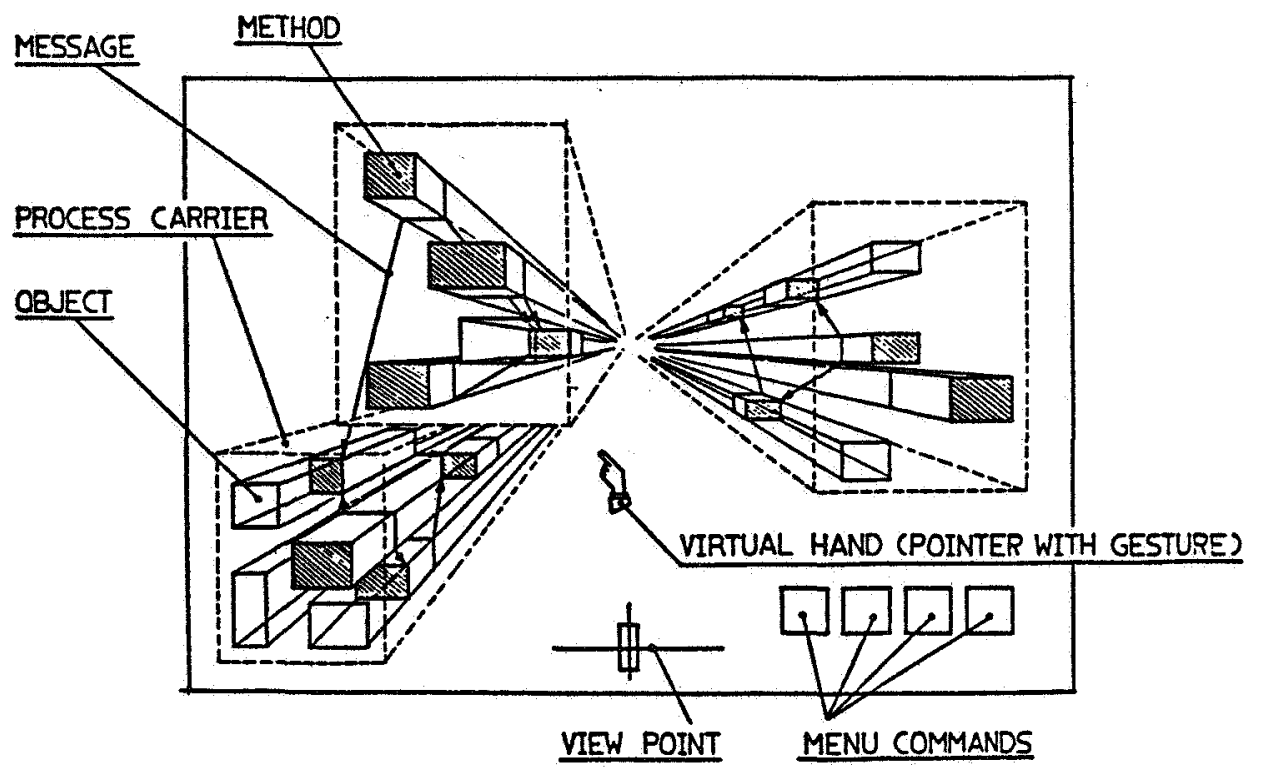

Figure 3. Example of visual 3D programming environment. 


\title{
HUMAN OPERATOR TRACKING PERFORMANCE WITH A VIBROTACTILE DISPLAY
}

\author{
Gideon F. Inbar \\ Technion - Israel Institute of Technology \\ Haifa , ISRAEL
}

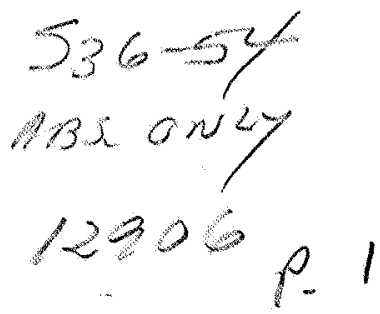

Vibrotactile displays have been designed and used as a sensory aid for the blind. In the present work the same $6 \times 24$ "Optacon" type vibrotactile display (VTD) was used to characterize human operator (HO) tracking performance in pursuit and compensatory tasks. The VTD was connected via a microprocessor to a one-dimensional joy stick manipulator. Various display schemes were tested on the VDT, and were also compared to visual tracking performance using a specially constructed photo diode matrix display comparable to the VTD.

Optimal performance, measured as minimal rms error between input and output signals, was achieved by dividing the VDT matrix into two $3 \times 24$ fields. On one side, l the reference signal was presented and on the second half the target position. The axial position on either half was generated by cycling between two adjacent 3 pin lines at $40 \mathrm{~Hz}$, moving up and down with the target, or reference signals. Pseudo random bandlimited signals, DC to $0.4 \mathrm{HZ}$ were used testing the performance of four subjects.

Results:

1. Very little training was needed by the subjects to learn to use the system and there was little improvement with time after the quick initial learning phase.

2. No significant differences were measured between pursuit and compensatory tasks, in contrast to the visual tracking experiment.

3. The calculated coherence function for both tasks was close to 1.0 at the above dynamic range.

4. The HO transfer function, after the exclusion of a fixed time delay, was a constant gain and constant phase at the above range.

5. An average of about $420 \mathrm{msec}$. time delay between the input and output signals was exhibited by three subjects while the fourth subject had almost double this delay. 
IMPLEMENTATION ISSUES FOR A COMPACT 6 DEGREE OF FREEDOM FORCE REFLECTING HANDCONTROLLER WITH CUEING OF MODES

\author{
Heidi Jacobus, Alan J. Riggs, Charles Jacobus, and Yechiel Weinstein \\ Cybernet Systems Corporation \\ Ann Arbor, Michigan
}

Teleoperated control requires a master human interface device that can provide haptic input and output which reflects the responses of a slave robotic system. The effort reported in this paper addresses the design and prototyping of a six degree-of-freedom (DOF) Cartesian coordinate hand controller for this purpose. The device design recommended is an XYZ stage attached to a three-roll wrist which positions a flight-type handgrip. Six degrees of freedom are transduced and control brushless DC motor servo electronics similar in design to those used in computer controlled robotic manipulators. This general approach supports scaled force, velocity, and position feedback to aid an operator in achieving telepresence. The generality of the device and control system characteristics allow the use of inverse dynamics robotic control methodology to project slave robot system forces and inertias to the operator (in scaled form) and at the same time to reduce the apparent inertia of the robotic handcontroller itself.

The handcontroller unit which was designed and simulated in the initial effort uses brushless DC servo motors, integral pancake resolvers, and limited gearing to produce an extremely simple and rugged mechanical design requiring minimal routine maintenance. The electronic control unit designed is a compact low power robotic driver which supports robotic manipulator systems needing up to nominally $1500 \mathrm{~W}$ of power from a personal computer sized package. The current control design, which is not multiple fault tolerant, can be extended in this area to make flight control or space use possible.

The proposed handcontroller will have advantages in space-based applications where an operator must control several robot arms in a simultaneous and coordinated fashion. It will also have applications in intravehicular activities (within the Space Station) such as microgravity experiments in metallurgy and biological experiments that require isolation from the astronauts' environment. For ground applications, the handcontroller will be useful in underwater activities where the generality of the proposed handcontroller becomes an asset for operation of many different manipulator types. Also applications will emerge in the Military, Construction, and Maintenance/Manufacturing areas including ordnance handling, mine removal, NBC (Nuclear, Chemical, Biological) operations, control of vehicles, and operating strength and agility enhanced machines. Future avionics applications including advanced helicopter and aircraft control may also become important.

1. The work reported here has been supported by NASA Johnson Space Center under contract NAS-9-18094.

2. Poster version. 


\title{
ON THE ROLE OF EXCHANGE OF POWER AND INFORMATION SIGNALS IN CONTROL AND STABILITY OF THE HUMAN-ROBOT INTERACTION
}

\author{
H. Kazerooni \\ University of Minnesota \\ Minneapolis, Minnesota
}

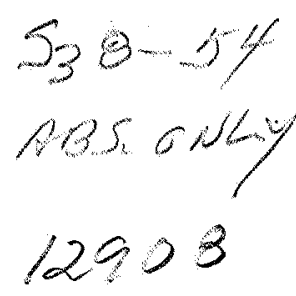

A human's ability to perform physical tasks is limited, not only by his ${ }^{1}$ intelligence, but by his physical strength. If, in an appropriate environment, a machine's mechanical power is closely integrated with a human arm's mechanical power under the control of the human intellect, the resulting system will be superior to a loosely integrated combination of a human and a fully automated robot. Therefore, we must develop a fundamental solution to the problem of "extending" human mechanical power. The work presented here defines "extenders" as a class of robot manipulators worn by humans to increase human mechanical strength, while the wearer's intellect remains the central control system for manipulating the extender. The human, in physical contact with the extender, exchanges power and information signals with the extender.

The aim is to determine the fundamental building blocks of an intelligent controller, a controller which allows interaction between humans and a broad class of computer-controlled machines via simultaneous exchange of both power and information signals. Traditionally, human interaction with active systems (self-powered machines) has been defined as the exchange of "information signals" only. For example, the human sends information signals to an electric mixer (an active system) by pushing a "start" button, but the human does not transfer power to the mixer and does not feel the actual load on the mixer blades. As in this example, the prevalent trend in automation has been to physically separated the human from the machine so the human must always send information signals via an intermediary device (e.g., joystick, pushbutton, lightswitch). Extenders, however are perfect examples of self-powered machines that are built and controlled for the optimal exchange of power and information signals with humans. The human wearing the extender is in physical contact with the machine, so power transfer is unavoidable and information signals from the human help to control the machine. Commands are transferred to the extender via the contact forces and the EMG signals between the wearer and the extender, thus eliminating the need for a joystick, pushbutton, lightswitch or keyboard. This allows the person to maneuver his hand more naturally. If "talking" is defined as a natural method of communication between two people, then we would like to communicate with a computer naturally by talking rather than by using a keyboard. The same is true here: if we define "maneuvering the hand" as a natural method of moving loads, then we would like to move only the hand to maneuver a load, as opposed to using a keyboard or joystick. The extender augments human motor ability without accepting any explicit commands: it accepts the EMG signals and the contact force between the person's arm and the extender, and the extender "translates" them into a desired position. In this unique configuration, mechanical power transfer between the human and the extender occurs because the human is pushing against the extender. The extender transfers to the human's hand, in feedback fashion, a scaled-down version of the actual

1. The pronouns "he," "his," and "him" used in this abstract are not meant to be gender-specific. 
external load which the extender is manipulating. This natural feedback force on the human's hand allows him to "feel" a modified version of the external forces on the extender.

Further clarification of the concept of transfer of power and information signals follows. The information signals from the human (e.g., EMG signals) to the computer reflect human cognitive ability, and the power transfer between the human and the machine (e.g., physical interaction) reflects human physical ability. Thus the information transfer to the machine augments cognitive ability, and the power transfer augments motor ability. These two actions are coupled through the human cognitive/motor dynamic behavior. The goal is to derive the control rules for a class of computer-controlled machines that augment human physical and cognitive abilities in certain manipulative tasks. 


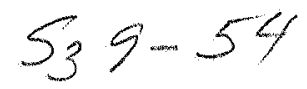

Myron W. Krueger

Artificial Reality

Vernon, Connecticut

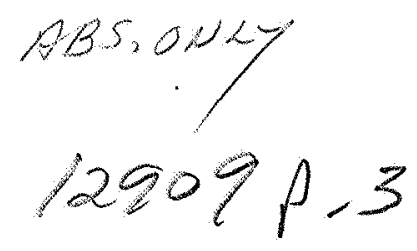

The current presumption is that is necessary to don goggles, gloves and a data suit to experience artificial reality. However, there is another technology that offers an alternative or complement to the encumbering techniques associated with NASA. In VIDEOPLACE, your image appears in a 2D graphic world created by a computer. The VIDEOPLACE computer can analyze a person's image in 1/30 second and can detect when an object is touched (Fig. 1). Thus, it can generate a graphic or auditory response to an illusory contact.

VIDEOPLACE technology exists in two formats: the VIDEODESK and the VIDEOPLACE. In the VIDEODESK environment, the image of your hands can be used to perform the normal mouse functions, such as menuing and drawing (Fig. 2). In addition, you have the advantage of multippoint control. For instance, you can use the thumbs and forefingers of each hand as control points for a spline curve (Fig. 3). Perhaps most important, the image of your hands can be compressed and transmitted to a colleague over an ISDN voice channel to appear on the remote screen superimposed over identical information. Likewise, the image of your colleague's hands can appear on both screens. The result is that the two of you can use your hands to point to features on your respective screens as you speak, exactly as you would if you were sitting together (Fig. 4).

In the VIDEOPLACE environment, you can interact with graphic creatures and the images of other people in other locations in a graphic world. Your whole body can be moved, scaled and rotated in real-time without regard to the laws of physics. Thus, VIDEOPLACE can be used to create a fantasy world in which the laws of cause and effect are composed by an artist (Fig. 5) (Fig. 6).

\section{REFERENCES}

Krueger, M. W. Computer Controlled Responsive Environments,Dissertation, University of Wisconsin, 1974.

Krueger, M. W. Responsive Environments, NCC Proceedings, 1977, 375-385. Krueger, M. W. Artificial Reality, Addison-Wesley, 1983.

Krueger, M. W. VIDEOPLACE-An Artificial Reality, SIGCHI 85 Proceedings, April 1985, 35-40. 


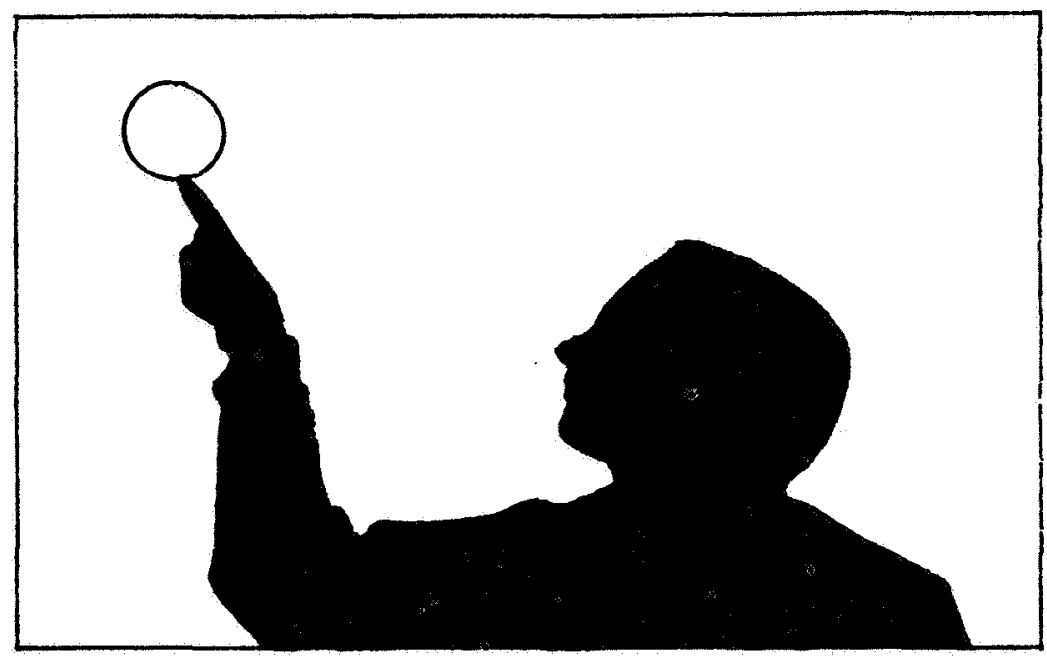

Figure 1. Video touch.

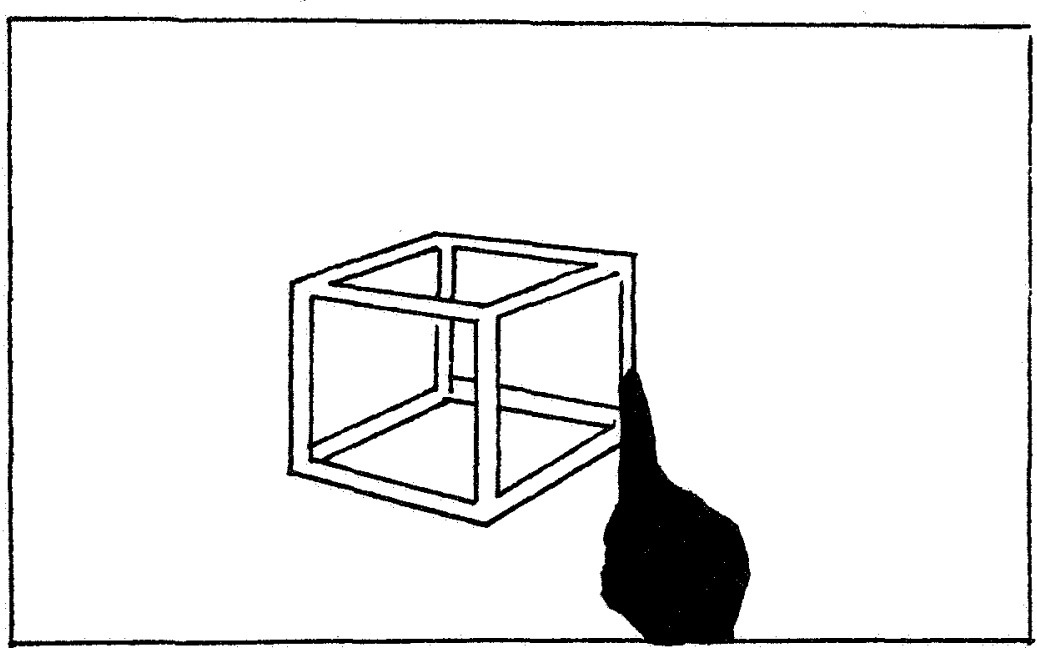

Figure 2. Videodesk drawing.

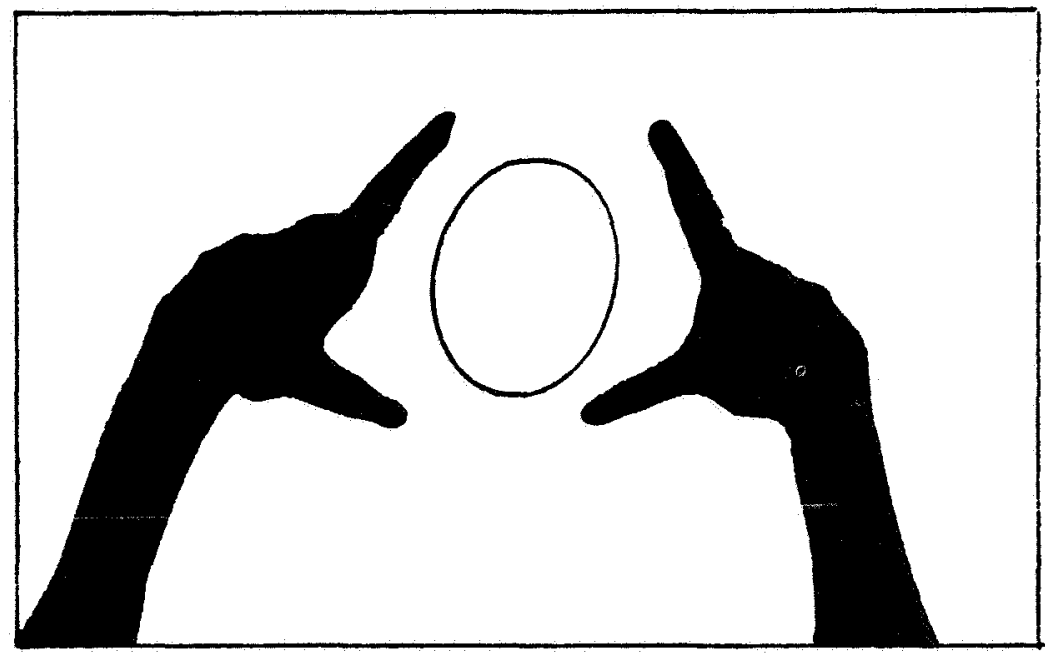

Figure 3. Multipoint control. 


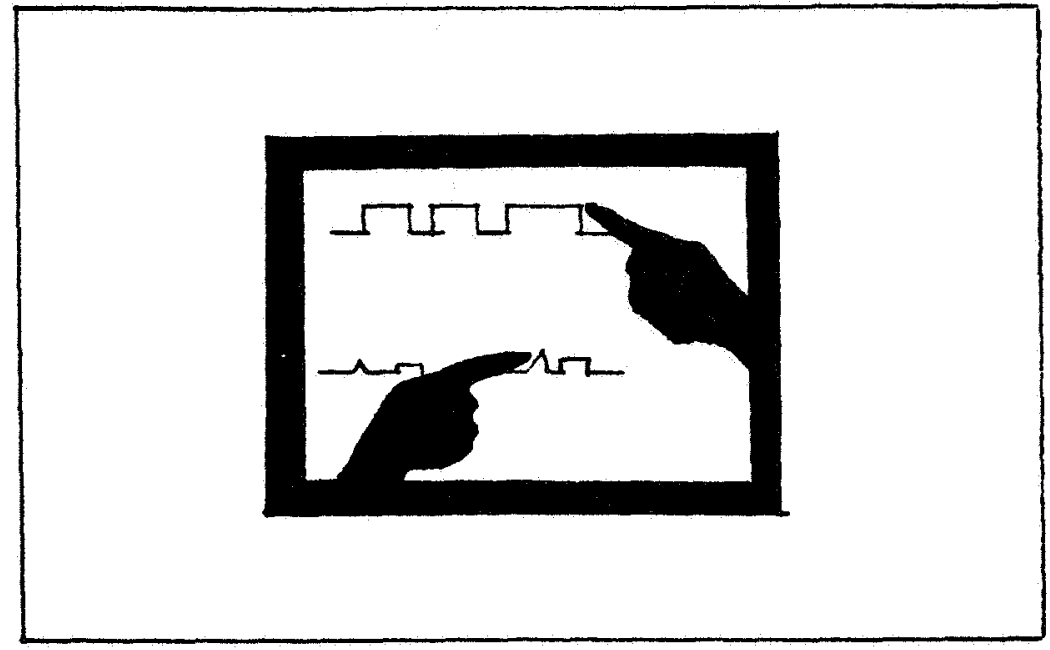

Figure 4. Telecommunication.

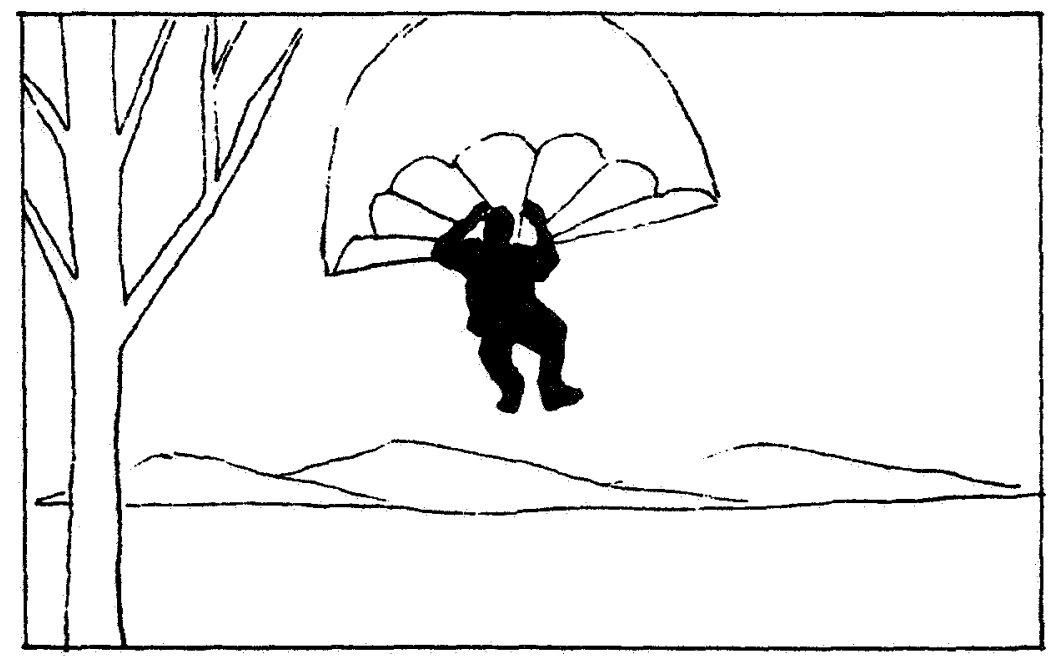

Figure 5. Landing in an artificial reality.

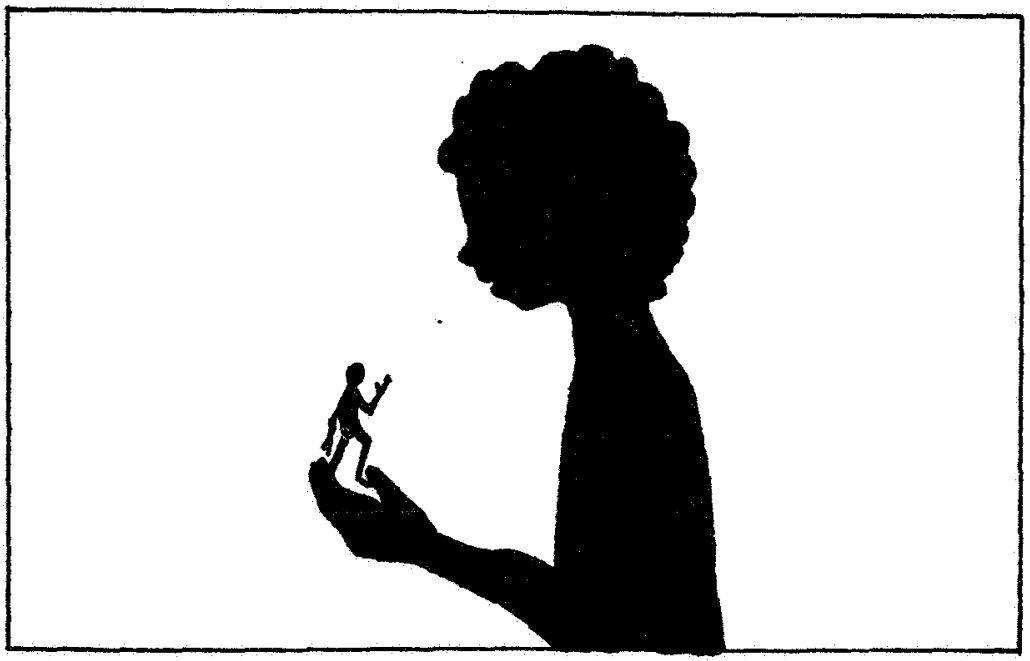

Figure 6. Parent and child size reversal. 


\title{
BINAURAL ROOM SIMULATION
}

\author{
H. Lehnert, J. Blauert, and W. Pompetzki \\ Ruhr Universitat Bochum \\ Bochum, FR GERMANY
}

In every-day listening the auditory event perceived by a listener is determined not only by the sound signal that a sound emits but also by a variety of environmental parameters. These parameters are the position, orientation and directional characteristics of the sound source, the listener's position and orientation, the geometrical and acoustical properties of surfaces which affect the sound field and the sound propagation properties of the surrounding fluid. A complete set of these parameters can be called an Acoustic Environment. If the auditory event perceived by a listener is manipulated in such a way that the listener is shifted acoustically into a different acoustic environment without moving himself physically, a Virtual Acoustic Environment has been created. Here, we deal with a special technique to set up nearly arbitrary Virtual Acoustic Environments, the Binaural Room Simulation. The purpose of the Binaural Room Simulation is to compute the binaural impulse response related to a virtual acoustic environment taking into account all parameters mentioned above.

One possible way to describe a Virtual Acoustic Environment is the concept of the virtual sound sources. Each of the virtual sources emits a certain signal which is correlated but not necessarily identical with the signal emitted by the direct sound source. If source and receiver are non moving, the acoustic environment becomes a linear time-invariant system. Then, the Binaural Impulse Response from the source to a listener's eardrums contains all relevant auditory information related to the Virtual Acoustic Environment. Listening into the simulated environment can easily be achieved by convolving the Binaural Impulse Response with dry signals and representing the results via headphones.

Our simulation system contains the following steps. First the parameters of the environment to be simulated are entered into the computer using a CAD program geometric data of the room and databases for acoustic data of the walls and the directional characteristics. Position and orientation of source and receiver are defined by local coordinate systems. Then, the positions and orientations of the virtual sound sources are computed by ray tracing or mirror imaging algorithms (refs. 1, 2, and 3). The signals of the virtual sources are derived by convoluting all impulse responses of the walls involved in the sound path of the virtual source. The binaural room impulse response is then computed by propagating the signal of each virtual source to the listener and convolving the result with the external-ear impulse response of the listener for the respective direction of incidence.

\section{REFERENCES}

1. J. Borish, "Extension of the image model to arbitrary polyhedra," J. Acoust. Soc. Am., Vol 75(6), pp. 1827-1836, June 1984. 
2. J. P. Vian et al., "Calculation of the room impulse response using a ray-tracing method," 12 th ICA, Vancouver Symposium, pp. 74-78, 1986.

3. M. Vorlander, "Ein Strahlverfolgungsverfahren zur Berechnung von Schallfeldern in Raumen (A ray-tracing program for the calculation of sound fields of rooms)," Acustica, Vol. 65, pp. 138-148, 1988 (in German). 


\section{ACTIVE LOCALIZATION OF VIRTUAL SOUNDS}

\section{$5 \% \%$ \\ $1050 \mathrm{H}$ \\ 12911}

\author{
J. M. Loomis, C. Hebert, and J. G. Cicinelli \\ University of California \\ Santa Barbara, California
}

We describe a virtual sound display built around a $12 \mathrm{MHz} 80286$ microcomputer and specialpurpose analog hardware. The display implements most of the primary cues for sound localization in the ear-level plane. Static information about direction is conveyed by interaural time differences and, for frequencies above $1800 \mathrm{~Hz}$, by head sound shadow (interaural intensity differences) and pinna sound shadow. Static information about distance is conveyed by variation in sound pressure (first power law) for all frequencies, by additional attenuation in the higher frequencies (simulating atmospheric absorption), and by the proportion of direct to reverberant sound. When the user actively locomotes, the changing angular position of the source occasioned by head rotations provides further information about direction and the changing angular velocity produced by head translations (motion parallax) provides further information about distance. Judging both from informal observations by users and from objective data obtained in an experiment on homing to virtual and real sounds, we conclude that simple displays such as this are effective in creating the perception of external sounds to which subjects can home with accuracy and ease. 


\title{
HUMAN-MACHINE INTERFACE HARDWARE: THE NEXT DECADE
}

\author{
E. A. Marcus \\ EXOS, Inc. \\ Lexington, Massachusetts
}

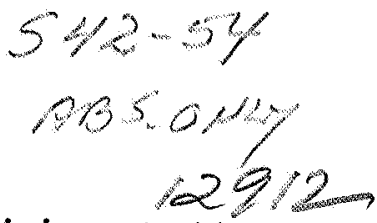

In order to understand where human-machine interface hardware is headed, it is important to understand where we are today, how we got there, and what our goals for the future are.

As computers become more capable, faster, and programs become more sophisticated, it becomes apparent that the interface hardware is the key to an exciting future in computing. One only has to look at $\mathrm{CAD}$ workstation hardware and the array of tablet, stylus, trackball, knob, and mouse devices which are combined with a maze of menus and function keys to understand the issues. These programs can create visual representations in 2 or 3-D, illustrate attributes of the object, such as stress and temperature distributions, or model and animate the object as it interacts with other objects. How can a user interact and control this seemingly limitless array of parameters effectively? Today, the answer is most often a limitless array of controls. The link between these controls and human sensory motor capabilities does not utilize existing human capabilities to their full extent.

Imagine if the developers of the first car had electronic controls at their disposal and decided to control all car functions through a tablet or an array of switches or dials. They might have argued against a steering wheel as follows, "If a steering wheel is used, both hands are completely occupied at all time. How would you control the speed, or any of the other functions?" The rebuttal might have been, "Use foot pedals which is natural due to experience with walking or cycling." The tablet advocate would have said, "No one will want to use their feet, it's just not natural. Even if people would try it, it wouldn't be smooth and it would damage the whole concept of the car." As you can see, if cars evolved this way, they would be a maze of dials, menus and controls which saturate human sensory capabilities. We'd probably have speed limits of $30 \mathrm{mph}$ due to the limitations in the reaction time as set by the interface hardware. Luckily, the car evolved to take more advantage of human capabilities.

To use the car analogy again, when the car was born, they didn't ask what a car in the year 2000 would look like and leave "hooks" and "scars" for improved technology. If they had, they would have been wrong, as few if any people are able to anticipate technology breakthroughs. They did, however, provide the best that was possible at the time and got the product out to as many people as possible and let time, experience, market demand, and technology innovation lead to the future.

Interface hardware for teleoperation and virtual environments is now facing just such a crossroad in design. Therefore, we as developers need to explore how the combination of interface hardware, human capabilities, and user experience can be blended to get the best performance today and in the future. 


\title{
COORDINATION OF HUMAN UPPER ARM AND FOREARM MOTION
}

\section{IN RAPID EXTENSIONS}

\author{
Mahmood Nahvi \\ California Polytechnic State University \\ San Luis Obispo, California
}

P

In many movements of the upper limb such as reaching, positioning, and diplacing the objects, the hand moves smoothly along a uni-directional planar trajectory with a bell-shaped speed profile.

Because of variability of the load, gravitational and velocity interaction forces between its segments, the dynamics of the arm during the motion is very complex. Motion of the upper arm and the forearm are coordinated to produce smooth movements despite such complex dynamics. This coordination constitutes an important organizational feature of arm movement. The present paper describes some experimental results related to the above and interprets their role in producing smooth motion of the hand.

Trajectories of the right upper limb in vertical plane and the simultaneous muscles' EMG activities were recorded for extensions of various amplitudes under four loads. The forearm trajectories are smooth with bell-shaped speed profiles. The upper arm trajectories may have bimodal speed profiles and three segments, resembling an inverted "Z." Segmentation is sharper for the points near the shoulder joint, and is accentuated by load. As one moves from the central points near the shoulder joint to the peripheral points near the hand, the three segments merge and result in smooth curves with single-peaked speed profiles. The three segments have a coherent time course and can be identified rather accurately and non-ambiguously.

The observed trajectories and the EMG patterns reveal an effective coordination strategy which utilizes the structure and the dynamics of the moving arm to produce smooth movement. 


\section{MANUAL DISCRIMINATION OF FORCE}

\author{
X. D. Pang, H. Z. Tan, and N. I. Durlach \\ Massachusetts Institute of Technology \\ Cambridge, Massachusetts
}
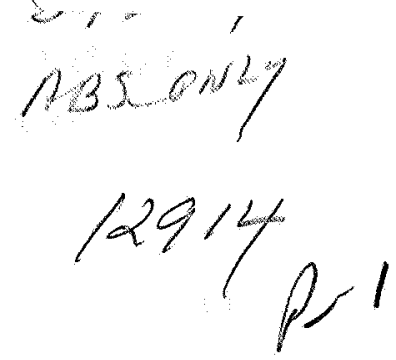

Optimal design of human-machine interfaces for teleoperators and virtual-environment systems which involve the tactual and kinesthetic modalities requires knowledge of the human's resolving power in these modalities. The resolution of the interface should be appropriately matched to that of the human operator. We report some preliminary results on the ability of the human hand to distinguish small differences in force under a variety of conditions. Experiments were conducted on force discrimination with the thumb pushing an interface that exerts a constant force over the pushing distance and the index finger pressing against a fixed support. The dependence of the sensitivity index $d$ ' on force increment can be fit by a straight line through the origin and the just-noticeable difference (JND) in force can thus be described by the inverse of the slope of this line. The receiver-operating-characteristic (ROC) was measured by varying the a priori probabilities of the two alternatives, reference force and reference force plus an increment, in one-interval, two-alternative, forced-choice experiments. When plotted on normal deviate coordinates, the ROCs were roughly straight lines of unit slope, thus supporting the assumption of equal-variance normal distributions and the use of the conventional $d$ ' measure. The JND was roughly $6-8 \%$ for reference force ranging from 2.5 to 10 newtons, pushing distance from 5 to $30 \mathrm{~mm}$, and initial finger-span from 45 to $125 \mathrm{~mm}$. Also, the JND remained the same when the subjects were instructed to change the average speed of pushing from 23 to $153 \mathrm{~mm} / \mathrm{sec}$. The pushing was terminated by reaching either a wall or a well, and the JNDs were essentially the same in both cases. 


\title{
IMPLEMENTATION OF A THREE DEGREE OF FREEDOM, MOTOR/BRAKE HYBRID FORCE OUTPUT DEVICE FOR VIRTUAL ENVIRONMENT CONTROL TASKS
}

\author{
Massimo Russo, Alfred Tadros, Professor Woodie Flowers, and Professor David Zelter \\ Massachusetts Institute of Technology \\ Cambridge, Massachusetts
}

The advent of high resolution, physical model based computer graphics has left a gap in the design of input/output technology appropriate for interacting with such complex virtual world models. Since virtual worlds consist of physical models, it is appropriate to output the inherent force information necessary for the simulation to the user. The detailed design, construction, and control of a three degree freedom force output joystick will be presented.

A novel kinematic design allows all three axes to be uncoupled, so that the system inertia matrix is diagonal. The two planar axes are actuated through an offset gimbal, and the third through a sleeved cable. To compensate for friction and inertia effects, this transmission is controlled by a force feedforward and a closed force feedback proportional loop. Workspace volume is a cone of 512 cubic inches, and the device bandwidth is maximized at $60 \mathrm{~Hz}$ for the two planar and $30 \mathrm{~Hz}$ for the third axis. Each axis is controlled by a motor/proportional magnetic particle brake combination fixed to the base. The innovative use of motors and brakes allows objects with high resistive torque requirements to be simulated without the stability and related safety issues involved with high torque, energy storing motors alone. Position, velocity, and applied endpoint force are sensed directly. Different control strategies are discussed and implemented, with an emphasis on how virtual environment force information, generated by the MIT Media Lab Computer Graphics and Animation Group BOLIO system, is transmitted to the device controller.

The design constraints for a kinesthetic force feedback device can be summarized as:

How can the symbiosis between the sense of presence. in the virtual environment be maximized without compromising the interaction task, under the constraints of the mechanical device limitations?

Research in this field will yield insights to the optimal human sensory feedback mix for a wide spectrum of control and interaction problems. A flexible research tool that is designed as an easily reproducible product prototype has been constructed to explore the variety of possible force interaction. 


\section{Introduction}

The advent of high resolution, physical model based computer graphics has left a gap in the design of input/output technology appropriate for interacting with such complex virtual world models. Visual display technology has improved dramatically, driven by developing communication through the most information rich sensory channel. Advances in stereographic displays and more proprioceptive input devices that contain higher degreee of freedom position information, such as full hand or body gesture recognition, still rely only on the visual sense. The user operates in a world of relative paralasys, not able to feel, grasp, touch, smell, or poke the objects that she interacts with.

Since virtual worlds are based on physical models, it is appropriate to output the inherent force information necessary for the simulation to the user. Before one commits large resources to the construction of such force output machines, the appropriate design constraints and problem definitions for simulated environment interactions must be addressed. The problem can be summarised as:

How can the symbiosis between the sense of presence in the virtual environment be maximised without compromising the interaction task, under the constraints of the mechanical device limitations?

Research in this field will yield insights to the optimal human sensory feedback mix for a wide spectrum of control and interaction problems: telepresence and supervisory control in remote manipulator tasks, advanced computer assisted engineering design tools, and surgical or flight simulators among many others. A flexible research tool to explore the variety of possible force interactions and is nevertheless designed as an easily reproducable product prototype has been constructed.

\section{Overview and Design Specification}

Traditional force output devices have concentrated on large, global movements, motivated by the scale of the teleoperated robot motion. In general, an analog slave sensor feedback signal servos the master joint motors. The geometric configuration and physical dimensions of the controlled manipulator and of the master are the same. In many cases, however, geometric equality is not necessary and can even hinder a particular successful task completion. Recently, in the flexible, unspecified tasks that are possible with virtual environments, the goal is to design the force output device so that its own dynamics are transparent and yet also can simulate as wide a frequency band of forces as possible. The range of motion used in interacting with a computer can be limited to a small surface or volume, one need only think of the small "mouse" interface device movement. The design of a force output device for virtual environments should maximize the device fidelity under the general constraints of size, cost, limited computation speed, and safety. These are the general constraints that drove the design of the presented force feedback interface. The design catagories are detailed below. 


\title{
Design Categories:
}

\author{
Engineering Development: \\ Design Simplicity: \\ Appearance \\ Limited Size \\ Robust \\ Versatile \\ Modular \\ Expandable \\ Safety \\ Difficulty of implementation \\ Technological base \\ Cost \\ Controllability: \\ Interface Transparency: \\ Can be counterbalanced (Electronically or Mechanically) Backdrivable \\ High DOF \\ Uncoupled axes \\ Low degree of computation: \\ Full State measurement \\ Integrated into system w/o control/graphic display interference \\ Limited control logic \\ High Position Resolution \\ Uncoupled axes \\ Closed or open loop control: \\ Full State measurement \\ Stability: \\ Stiff \\ High natural frequency \\ Human-Device Interaction: \\ Secondary Function Control \\ Non-fatiguing \\ Safety \\ Human arm limitations never exceeded \\ Interface Transparency \\ Variable force feedback ratio
}

The described force output joystick incorporates linear but no rotational force output. Many manual tasks in which force information is of utmost importance and that are completed with the use of tools can be limited to three linear degrees of freedom. The metaphor used as a design guideline, which allows for a tractable problem within the design requirements, is a tool endpoint force simulator. To maintain design simplicity and limit the size without compromising device versatility, the joystick endpoint is limited to the three degrees of linear motion. It can be upgraded to include a 
small three degrees of freedom torque output mechanism, so a full 6 degrees of freedom force and torque output could be realised.

Any point contact can by modulated according to the characteristics imposed by the simulation. The joystick endpoint is a single probe with which to explore the virtual computer space. How effective such an approach is in transmitting tactile information is a matter of ongoing research (Minsky). Full, high resolution tactile displays have a large number of independent degrees of freedom, determined by the size and resolution of the display. Each tactile "pixel" must be independently controlled, so devices of this kind are difficult to implement. Examples include Braille machines for the blind, which are the state of the art for such devices, and it is an open question if their resolution is sufficient for effective, more general representation tactile displays.

A fine tool is not grasped with the whole hand, but rather like a pen between one's fingers. The force magnitude in such and interaction is much lower than in full arm movement. The restriction to finer forces and motions, which is appropriate for the limited workspace requirement of the human arm and hand, allows the joystick to be constrained in size since the actuator brakes and motors can have lower torque ouputs. Also, the smaller motors more easily meet the safety requirements and the limitation to three degrees of freedom decreases the difficulty of implementation and cost. The three degrees of freedom can be chosen such that the axes are perpendicular and all the actuators are attached to ground, thus eliminating cross coupling between the endpoint principal directions and the actuator torques. Rotary, no torque, ripple, low inertia motors and proportional magnetic particle brakes with a simple cable transmission for the third axis, and most of the sensors are readily available from existing technology. Only a three axis linear load cell of sufficiently small size and mass had to be specifically designed and constructed.

\section{Control Requirements}

The detailed mechanical design of the joystick is dominated by the control requirements of the simulation. To both impose the arbitrary simulation dynamics onto the joystick endpoint and minimize user fatigue when the joystick is used as a simple position input device, the actual characteristic or plant dynamics should not interfere with the simulation. This interface transparency is from the perspective of the user interacting with the joystick. One input to the plant is the force exerted by the user on the endpoint. The simulation, however, controls the desired dynamics through the input signals to the motors and brakes. The control strategy needs to maintain the simulation dynamics under varying user force inputs, or under the changing coupling characteristics between the user's arm and plant dynamics. The simulation essentially modulates the brake and motor signals so as to match the endpoint impedance or effective mass, stiffness, and damping characteristics to that of the virtual dynamics. The mechanical design maximizes the inherent device transparency and so minimized the control effort needed to backdrive the joystick. It has a bandwidty of $60 \mathrm{~Hz}$ for the gimbal driven axes and $30 \mathrm{~Hz}$ for the cable drive. Because of closed loop force control stability issues, open loop backdriveability is obtained wherever possible. This necessitates a direct drive between the brakes, motors, and joystick endpoint to minimize stiction, viscous friction and effective actuator inertia, which are all amplified by a transmission ratio. Direct drive in turn requires large motors for a significant torque. All the motors and brakes are fixed to ground and thus their weight and reaction torques need not be compensated for. Because of the much higher torque output of the brake for a given size and power input, any high torque requirements of the simulation will be met by the brake, an 
inherently passive, no energy storing and therefore safe torque device. Thus, both backdriveability and output force are maximized within the size and safety constraints.

No known research has been conducted in the appropriate strategy for hybrid, independent proportional brake and motor control. This novel combination promises to yield new methods of increasing stability and safety bound in actively controlled mechanisms without decreasing the closed loop device stiffness. The control of brakes necessitates a direct measurement of the contact force between the endpoint and user. Both for this reason and to actively backdrive the R-axis transmission, a loadcell 3-axis force sensor is implemented in the device.

The actual joystick endpoint position, velocity, and force are necessary to determine the characteristics of the interface dynamics. The servo loop sampling frequency is limited by the bandwidth of the simulated environment. As all the joystick control must operate in real time, the degree of computation lag in the control loop must be minimized. To achieve this and to eliminate quantization and noise errors at low velocities introduced by digital differentiation, both motor/brake shaft position and velocity are measured directly. The endpoint position and velocity can then be found through a simple matrix position and Jacobian transformation. The joystick linkage is chosen such that all actuator induced endpoint velocities are perpendicular. The axes are therefore uncoupled and any actuator exerts a force in one of the endpoint principle directions. The system inertia matrix is thus only diagonal, though time variant.

\section{Future Work}

Some simple texture and object simulation code has been implemented successfully. Present research efforts are concentrated on expanding the complexity of a real-time virtual world interaction by utilising the physically based graphics animation package in the MIT Media Lab Computer Graphics and Animation Group. Various studies of the effect of multiple sensory interaction on realism in simulations and the associated mechanical device complexity cost are proposed, with the present device as a research tool to quantify the effect of force information in successful task completion and enhanced apparent realism. 


\title{
HUMAN FACTORS REQUIREMENTS FOR TELEROBITIC COMMAND
} AND CONTROL: THE EUROPEAN SPACE AGENCY EXPERIMENTAL

\section{PROGRAMIME}

\author{
Robert J. Stone \\ The National Advanced Robotics Research Centre \\ Salford, United Kingdom
}

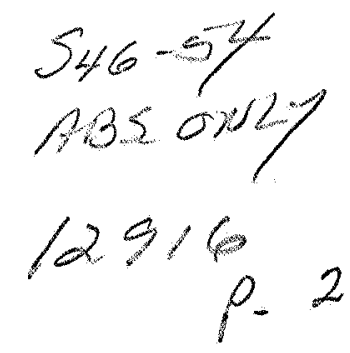

Space Telerobotics research, performed under contract to the European Space Agency (ESA), concerning the execution of human factors experiments, and ultimately leading to the development of a telerobotics test bed, has been carried out since 1985 by a British Consortium consisting of British Aerospace, the United Kingdom Atomic Energy Authority and, more recently, the UK National Advanced Robotics Research Centre. The principal aim of the first study of the series was to derive preliminary requirements for a teleoperation servicing system, with reference to two mission model scenarios. The first scenario introduced the problem of communications time delays, and their likely effect on the ground-based operator in control of a manipulator system on board an unmanned servicing vehicle in Low Earth Orbit. In the second scenario, the operator was located on the NASA Orbiter aft flight deck, supervising the control of a prototype manipulator in the "servicing" of an experimental payload in the cargo bay area. Human factors analyses centred on defining the requirements for the teleoperator workstation, such as identifying basic ergonomic requirements for workstation and panel layouts, defining teleoperation strategies, developing alphanumeric and graphic screen formats for the supervision or direct control of the manipulator, and the potential applications of expert system technology.

The second study for ESA involved an experimental appraisal of some of the important issues highlighted in the first study, for which relevant human factors data did not exist. Of central importance during the second study was the issue of communications time delays and their effect on the manual control of a teleoperated manipulator from a ground-based command and control station. An experimental test bed, based around ASEA Irb6 and 60 robots was developed, incorporating a general purpose robot controller, a reconfigurable operator workstation and a modular task box. The programme was divided into a series of short pilot studies and two principal experiments. The aim of the pilot studies was to optimise the characteristics of hand controllers and visual feedback conditions prior to their more formal evaluation and comparison in the later experiments. The experiments were conducted using standard psychological paradigms, involving human performance comparisons over a range of 3 and 6 degree-of-freedom hand controllers, with TV and graphics displays in various configurations. Although a rigorous experimental approach was adopted during Teleoperation and Control Study 2, it was evident that this approach suffers a number of limitations. The most critical concerns the extent to which the data and conclusions from this study can be generalised to future scenarios. Also, it is recognised that the performance measures of time and accuracy alone were not satisfactory, as they each provided only an overall index of performance for a given trial, not an analytical index of performance within the trial. Clearly, more analytical techniques to analyse operator performance in detail are required, together with a methodological framework in which all techniques can be used to judge the effect of "cost" of a particular technology, not only on human 
teleoperation performance, but also on the total telerobotic system, with the future aim of defining operational procedures.

Teleoperation and Control Study 3 is investigating a new technique - console or performance "logging"- for the measurement of teleoperation performance to complement those measures used in the second study, such as primitive and whole-task timings, handling errors, debriefing and subjective workload records (NASA TLX), video and audio recordings. Instrumenting the human-system interface to record and store both multiple gross actions and much finer keystroke-level parameters should provide a more detailed insight into the incidence, cause and effect of interactions between various control activities, and assist the experimenters in analyzing those control behaviours of too fine a nature to.be extracted from video records alone (such as fine individual joystick motions). Such a keystroke level of analysis should provide data suitable for future performance modelling activities in the area of telerobotic command and control.

Included among the parameters to be recorded in Study 3 are all robot control joystick axes, all additional joystick-mounted controls (where used), all panel-mounted switches, all camera joystick controls, all direct voice input utterances (incidence of onset only), all robot motions, as resolved at the end effector (i.e., translation and rotation), elapsed time (pulse code modulated), and experimenter-induced event markers. Areas of interest for the analysis work include an appraisal of individual strategies and their relation to task success, across-condition comparisons of control characteristics (e.g., when stereo vision or predictor displays are used in the experimental designs), error history monitoring (i.e., analysis of "lead-up" events), analysis of discrete event changes (e.g., the effects of discrete time delay changes, or selections of new robot control frames), and possibly an appraisal of the effects of controller "physical" characteristics (e.g., axis cross-talk, deadband, breakout forces). Finally, there are a number of outstanding questions to be addressed with regard to the use and development of the data logging system described above. For instance, is it desirable and even possible to use the data to generate metrics of performance suitable for complementing experimental and subjective results? Do other forms of control data (other than onset, direction and duration of control inputs) exist that are appropriate for logging? How can the concept be extended to record performance-related parameters other than control inputs (such as visual search, attention, mental workload, etc.)? Can a data logging system provide a useful insight into the operational cost of remote space operations? Answers to these questions will become clearer, following the use of the logging system during the experimental phase of the third contract, scheduled for the Summer of 1990. 


\title{
VERDEX: A VIRTUAL ENVIRONMENT DEMONSTRATOR FOR REMOTE DRIVING APPLICATIONS
}

\author{
$542-5 y$ \\ Robert J. Stone \\ The National Advanced Robotics Research Centre \\ Salford, United Kingdom

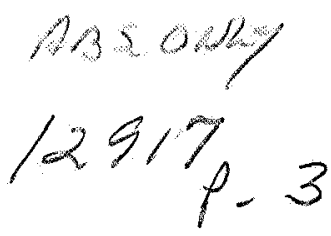

The National Advanced Robotics Centre was founded in June 1988, by the United Kingdom's Department of Trade and Industry and 11 industrial and academic shareholding organisations. The Centre acts as a focal point for the development of the core technologies which facilitate the next generation of robots, and is geared toward projecting the UK to the front of this international arena. One of the key areas of the Centre's enabling technologies research programme is that of the humansystem interface, phase 1 of which started in July 1989 and is currently addressing the potential of virtual environments to permit intuitive and natural interactions between a human operator and a remote robotic vehicle. The aim of the first 12 months of this programme (to September, 1990) is to develop a virtual human-interface demonstrator for use later as a test bed for human factors experimentation. This presentation will describe the current state of development of the test bed, and will outline some human factors issues and problems for more general discussion.

In brief, the virtual telepresence system for remote driving has been designed to take the following form. The human operator will be provided with a helmet-mounted stereo display assembly, facilities for speech recognition and synthesis (using the Marconi Macrospeak system), and a VPL DataGlove Model 2 unit. The vehicle to be used for the purposes of remote driving is a Cybermotion Navmaster K2A system, which will be equipped with a stereo camera and microphone pair, mounted on a motorised high-speed pan-and-tilt head incorporating a closed-loop laser ranging sensor for camera convergence control (currently under contractual development). It will be possible to relay information to and from the vehicle and sensory system via an umbilical or RF link. The aim is to develop an interactive audio-visual display system capable of presenting combined stereo TV pictures and virtual graphics windows, the latter featuring control representations appropriate for vehicle driving and interaction using a graphical "hand," slaved to the flex and tracking sensors of the DataGlove and an additional helmet-mounted Polhemus IsoTrack sensor.

Developments planned for the virtual environment test bed include transfer of operator control between remote driving and remote manipulation, dextrous end effector integration, virtual force and tactile sensing (also the focus of a current ARRL contract, initially employing a 14-pneumatic bladder glove attachment), and sensor-driven world modelling for total virtual environment generation and operator-assistance in remote scene interrogation. 


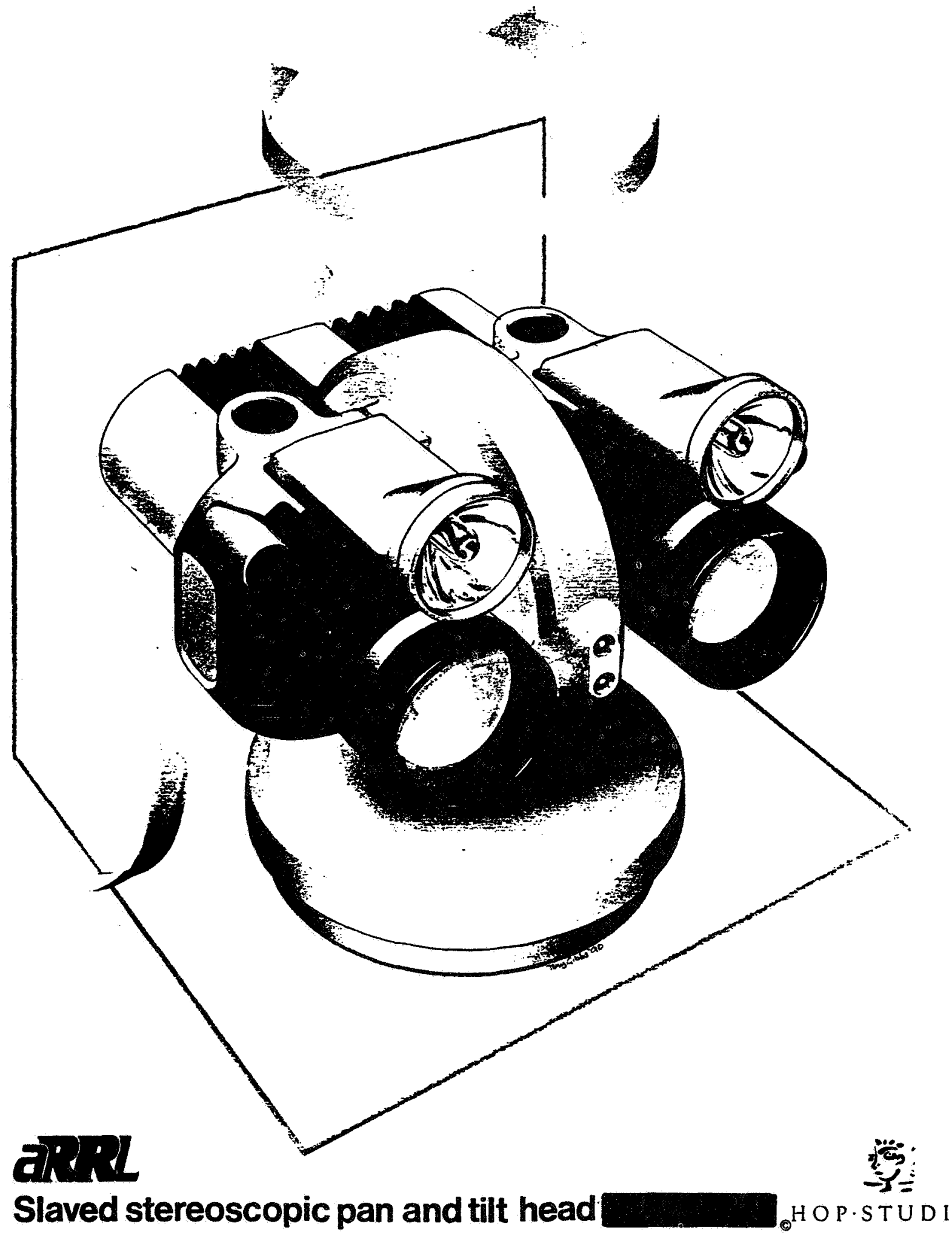




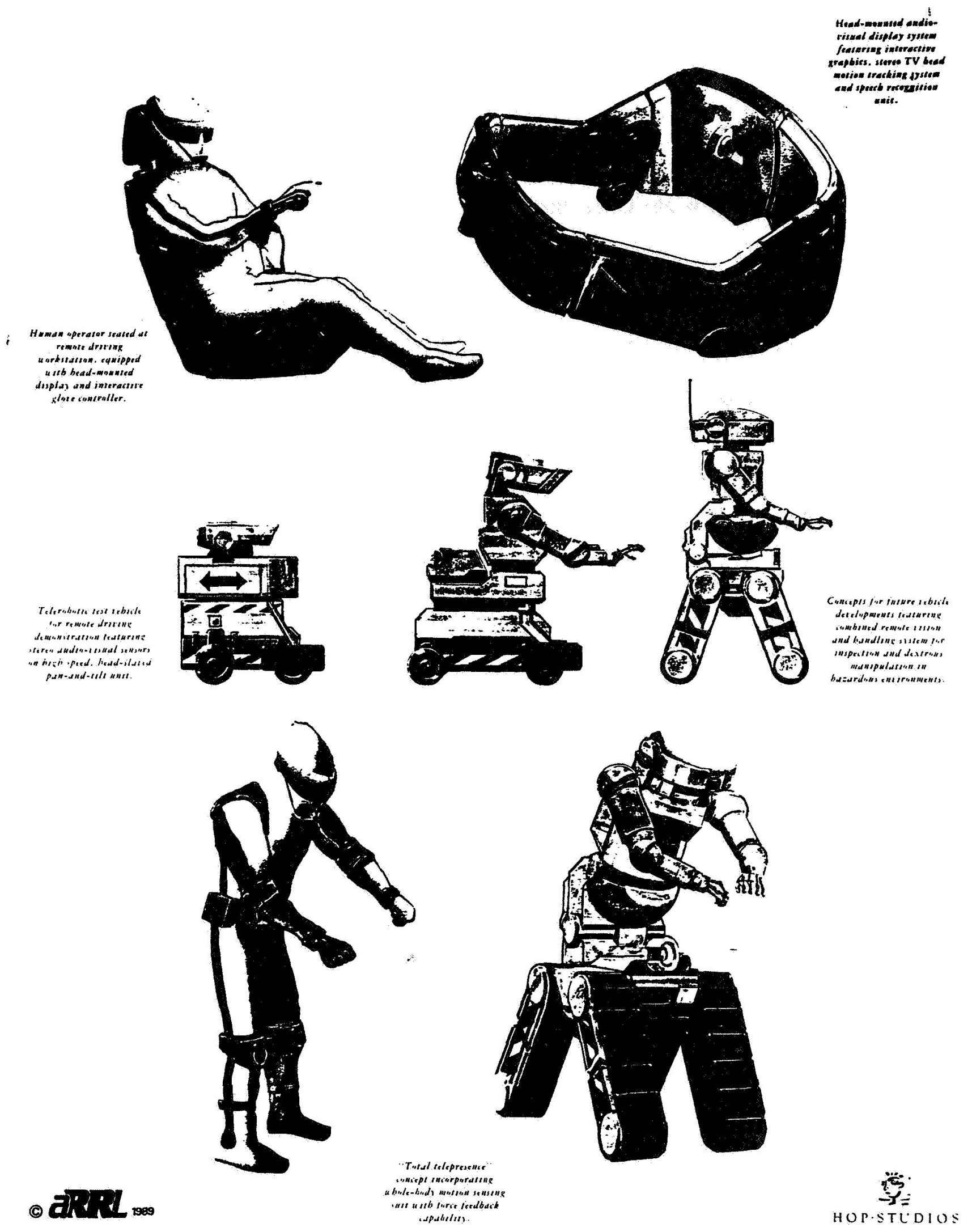

VIRTUAL MMI FOR ADVANCED ROBOTIC TELEPRESENCE 


\title{
DESTABILIZING EFFECTS OF VISUAL ENVIRONMENT MOTIONS SIMULATING EYE MOVEMENTS OR HEAD MOVEMENTS
}

\author{
K. D. White, D. Shuman, 1 J. H. Krantz, ${ }^{2}$ C. B. Woods, and L. A. Kuntz \\ University of Florida \\ Gainesville, Florida
}

Teleoperators are the humans who control devices from a "distance." This distance might be extreme physical separation, as in remote assembly of Space Station Freedom by ground-based personnel. But the distance could be effective distance of scale, for the manipulation of microscopic structures like single living cells or the components within an integrated circuit chip, or even the conceptual distance of safety, where the devices effect their actions inside a nuclear reactor's core or in the ocean's depths. Virtual environments refer to the synthesized realities that can be generated by various types of computerized displays, not only visual displays but also acoustic, tactile, and forcereflective displays. Video games are common but usually limited examples of virtual worlds. An implicit theme is telepresence, a term whose definition is imprecise. It involves the use of virtual environments to improve the efficiency of teleoperators by giving them a compelling sense of "being where the action is." Telepresence operations seem more natural and facile, and thus more easily trained, than other possible models for the human-computer interface. Consider a complex assembly task, controlled by wearing a sensor garment that enslaves a robotic arm to mimic the user's own arm movements, and visualized on video and felt by tactile and force-reflective feedbacks; versus, its control by typing code into a keyboard with feedback via numerical tables. Telepresence seems to endow the user with such a robust mental model that he or she becomes absorbed into the synthetic reality as though absorbed into a vivid dream.

The primary challenge for reduction to practice is to develop a paradigm for human-computer interaction that will enable telepresence to be implemented reliably. The natural movements of the human operator, such as the ability to "look around" in the virtual world, provide an important component for this paradigm inasmuch as the consistency of the virtual world can be enhanced by its enslavement to sensed head or eye movements of the human user.

In the present paper, we explore effects on the human of exposure to a visual virtual environment which has been enslaved to simulate the human user's head movements or eye movements. Specifically, we have studied the capacity of our experimental subjects to maintain stable spatial orientation in the context of moving their entire visible surroundings by using the parameters of the subjects' natural movements. Our index of the subjects' spatial orientation was the extent of involuntary sways of the body while attempting to stand still, as measured by translations and rotations of the head. We also observed, informally, their symptoms of motion sickness.

\footnotetext{
1 Now at Agricultural Research Service, U.S. Department of Agriculture.

${ }^{2}$ Now at Honeywell Phoenix Techology Center.
} 


\section{METHODS}

\section{Subjects}

A total of 93 university students or staff, 50 males and 43 females ranging in age from 18 to 43 years old, participated as unpaid volunteers in one of four experiments. Some students received course credit. Subjects were prescreened for medical histories (self-report) and any with possible vestibular defects were excused. Any subjects requiring refractive correction used their own prescribed lenses.

\section{Apparatus and Procedures}

Head Position Sensing. An acoustic timing principle was used to locate in space the headgear worn by each subject. This lightweight headgear contained two click sources, the wavefronts from which were detected with microsec timing resolution at each of four microphones attached to the ceiling of the testing room. Software algorithms for windowing the microphone outputs and performing redundancy checks, then implementing solutions of the Pythagorean theorem, permitted each click source to be located in space with translation precision below $1 \mathrm{~mm}$ at a sampling rate of $50 \mathrm{~Hz}$ maximum. Resolution of the rotation angle for the line connecting the two click sources was below $0.2 \mathrm{deg}$ ( $25 \mathrm{~Hz}$ maximum sampling rate). The headgear configuration did not permit resolution of rotations around the line connecting the click sources (pitch), but did permit resolution of the other five degrees of freedom for head movements.

Visual Environment. Each subject stood near the axis of a vertical hemicylindrical screen onto which was projected a pattern of vertical stripes. This pattern subtended in excess of $180 \mathrm{deg}$ of azimuth and $120 \mathrm{deg}$ in elevation. The instantaneous azimuth positioning of the pattern was under software control with a resolution of approximately $0.04 \mathrm{deg}$ at a maximum update rate exceeding $1 \mathrm{KHz}$. Impedances within the projection system were compensated for via nonlinear control signals when motions simulating saccadic eye movements were employed. In those particular experiments, the control signals were precomputed to reduce lag time to below $1 \mathrm{msec}$. When motions enslaved to the subject's head movements were employed, it was impractical to precompute the control signals. Thus, there was an additional lag time for computation of about $150 \mathrm{msec}$ and the update rate was reduced to $10 \mathrm{~Hz}$.

Simulations of Saccadic Eye Movements. Two small fixation lights rear-projected onto the surrounding screen could be alternated to direct horizontal saccades of particular azimuth extent $(0.5,1$, $2,3,4,6,8$, or $16 \mathrm{deg}$ ). Motions of the pattern front-projected onto the screen had characteristics of average velocity and motion duration similar to those for each corresponding saccade extent. ${ }^{3}$ In two experiments, the saccade-like stimulus motions occurred independent of the subject's eye movements. In another experiment, the stimulus motions were triggered by subject eye velocity, derived from the electro-oculogram, when the eye had moved 15-20 arcmin from its initial fixation during a guided voluntary saccade.

\footnotetext{
${ }^{3}$ A. T. Bahill, M. R. Clark \& L. Stark, Mathematical Biosciences, 24, 191-204 (1975).

Partially supported by a grant from the National Eye Institute to KDW (R23 EY03640), and by an NSF graduate fellowship to JHK.
} 
Head Movement Feedback Conditions. Each sample of the head's fore/aft and left/right translations plus head yaw angle permitted real time calculation of an equivalent azimuth change for the surrounding visual stimulus. This equivalent azimuth, modified by a gain factor that was the parameter selected for study, controlled motion of the visual stimulus in this experiment. The gain factors used were $+2,+1.5,+1,+0.5,0,-0.5,-1,-1.5$, and -2 . The gain of +1 , for example, could serve in principle to keep the stimulus "straight ahead" by its faithfully tracking the head's movements. Larger gains moved the stimulus farther and faster than would accurate head tracking; smaller gains moved the stimulus less far and more slowly. Zero gain made the stimulus remain still as in the natural environment. Negative gains reversed the left/right correspondence between head movements and stimulus motions.

\section{Data Analysis}

Control Conditions. Although the stability of a standing human has been modelled as an inverted pendulum, since the body's center of gravity is above its support base, the problem with such a model is that the human body is not mechanically rigid. Rather, it is a jointed mechanism controlled by over 400 muscles and several sensory feedback loops, with correspondingly complex dynamics. Furthermore, people grow to various heights, weights and distributions of body mass; with various extent of conditioning for their musculature; and with varied skills of sensorimotor integration. Individual variations in the body's dynamics are, therefore, prominent and, as a consequence, we rely on the use of experimental designs allowing each individual to serve as his/her own control.

Also, it is impractical to open the feedback loops from proprioceptive/kinesthetic or vestibular senses but it is relatively easy to alter those loops by asking the subject to stand only on one foot or else on a compliant surface. As reported in the results below, there are multiple techniques for controlling the visual feedback loop within each subject, including: (a) injecting noise via random motions of the visual environment, (b) stabilizing the environment via motion feedback from head movements, (c) eliminating contour from the visual environment, or (d) eliminating visibility by turning off the lights and (e) by closing the eyes.

Within-Subject Figure of Merit for Body Stability. There is no consensus in the literature for a representative figure of merit to describe such complicated behavioral dynamics as those under consideration. We have not been satisfied, in general, with time-domain analyses such as average movement velocity or RMS position error, since these statistical summaries tend to obscure details of the responses which we find to be correlated with experimental manipulations. We have, however, found one particular form of time-domain analysis to be useful in certain circumstances, and that form is time-locked response averaging. We plan to explore in future work the more general utility of a "chaotic" time-domain (phase-sensitive) analysis.

We have enjoyed considerable success in accounting for individual variations among subjects by using frequency-domain analysis, however. Specifically, each subject is measured: (a) under control conditions to determine his/her individual power spectral density (PSD) functions for body movements over time, and (b) under experimental conditions, to determine any changes in the PSD functions induced experimentally. The ratio between PSD functions (a) and (b) is the gain, as a 
function of the frequency, induced by the experimental manipulation. We find these gains to be relatively consistent betwen individuals (standard errors of measurement typically less than $1 \mathrm{~dB}$ ).

\section{RESULTS}

Figure 1 shows sample records of two subjects' lateral head movements while viewing moving surroundings (middle two traces). For the top trace, the motion of the stimulus was saccade-like, while for the bottom trace, the stimulus tracked the subject's head movements (gain $=+1.5$ ). These sample records have been displaced vertically for clarity.

The top trace of Figure 1 shows typical lateral sways made by subject $S 1$ when the entire visible surroundings suddenly moved with the velocity $(66 \mathrm{deg} / \mathrm{sec})$ and duration $(30 \mathrm{msec})$ characteristic of a 2 deg saccadic eye movement. Note the relatively pronounced fluctuations in head position. By a comparison against the second trace, S 1's lateral sways in still surroundings, it is clear that the stimulus motion exerts a strong destabilizing effect.

The bottom trace of Figure 1 shows lateral sways made by subject $S 2$ while the azimuth position of the visible stimulus tracked S 2's own head movements. By comparison against the third trace, subject S 2's lateral sways in still surroundings, the destabilizing effect of head tracking is apparent. Individual variations in stability can be noted by comparing the third trace (S 2) against the second trace (S 1), sways made in still surroundings.

Figure 2 depicts averaged yaw movements (i.e., head rotations) made in reponse to saccade-like motions of the stimulus, plotted as a function of time after the motion began. Each plotted point is based on 10 repetitions per subject times 18 subjects, or the average of 180 measures of head yaw angle per condition. The measures were carried out in three conditions: (1) saccade-only, the subject

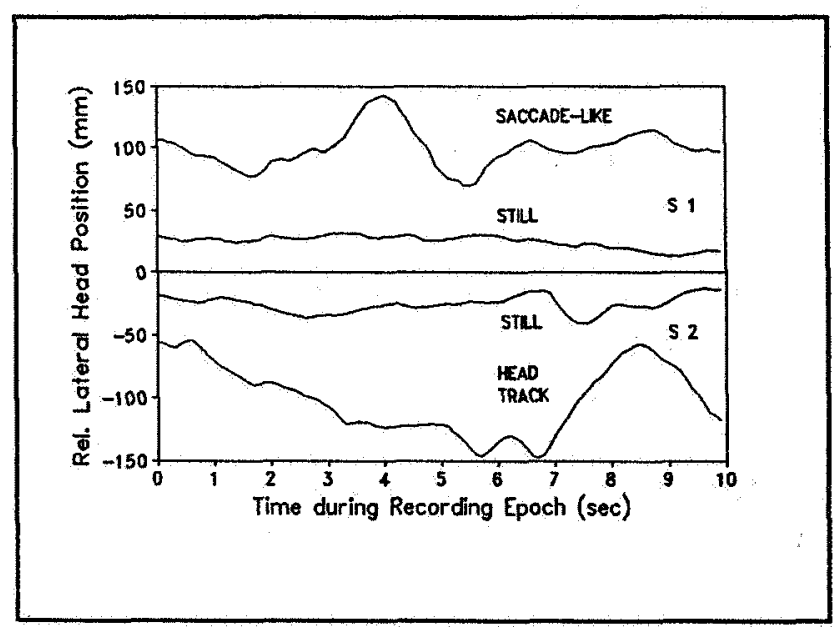

Figure 1. Sample records of head movements in still vs. moving environments (saccade-like or head tracking motions), for two subjects. 


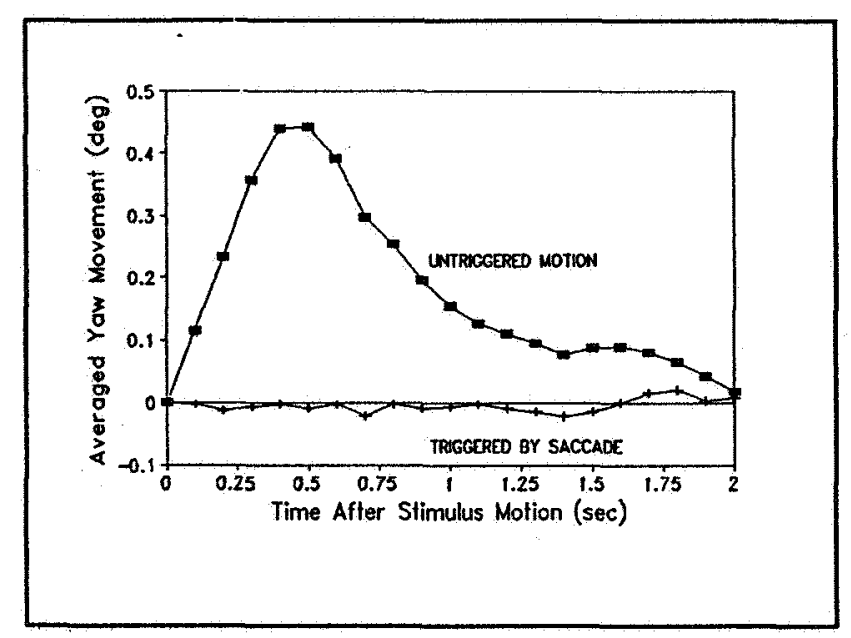

Figure 2. Response averages showing head yaw elicited by saccade-like stimulus motions. Motions triggered by saccades are less effective than untriggered motions for eliciting these responses.

moved their eyes as directed by fixation lights but there was no additional motion of the stimulus, (2) motion triggered by the saccade, in which a saccade-like stimulus motion took place while the subject's eyes were moving in a saccade, and (3) untriggered motion, in which a saccade-like stimulus motion briefly preceded ( $<100 \mathrm{msec})$ the subject's execution of a saccadic eye movement. In the saccade-only condition, subjects showed a slight tendency to yaw the head as well as to move the eyes. Average head yaw for saccade-only is taken as the baseline in this plot. This baseline motion is subtracted from the head yaws in response to stimulus motions.

It is clear in Figure 2 that untriggered saccade-like stimulus motions elicit a small but reliable yaw (the peak at $0.5 \mathrm{sec}$ represents 10 standard errors difference). This may be qualitatively similar to the lateral head translations illustrated in the top trace of Figure 1. However, Figure 2 also shows that the effect of the saccade-like stimulus motions can be lessened dramatically by causing them to coincide with the subject's own voluntary saccades. In this latter case, possibly a signal generated internally by the subject's oculomotor system (viz., corollary discharge or efference copy) alters the consequences that otherwise accompany the stimulus motion.

Figure 3 is a plot derived from fast Fourier transforms of sway records analogous to those shown in Figure 1. For the plot shown here, the power spectral density function (PSD) for each subject was obtained in still surroundings and this was taken as the baseline for that individual. PSDs for that subject under other conditions of stimulation were divided by his/her own baseline PSD. This resulted in gain profiles ( $\mathrm{dB}$ relative to baseline) as a function of sway frequency $(\mathrm{Hz})$. Each point on Figure 3 represents the average gain across 24 subjects ( 18 for saccades condition) within a frequency band $0.5 \mathrm{~Hz}$ wide. Typical standard errors associated with each point are under $1 \mathrm{~dB}$.

The six curves shown in Figure 3 depict the effects on subjects' stability of (1) closing their eyes, (2) eliminating visible contour (subjects wore diffusing goggles), (3) eliminating illumination (subjects wore opaque goggles, (4) moving the azimuth of the surroundings to track the subject's head movements (gain \pm 1.0 ), (5) moving the azimuth of the surroundings randomly, and (6) the 


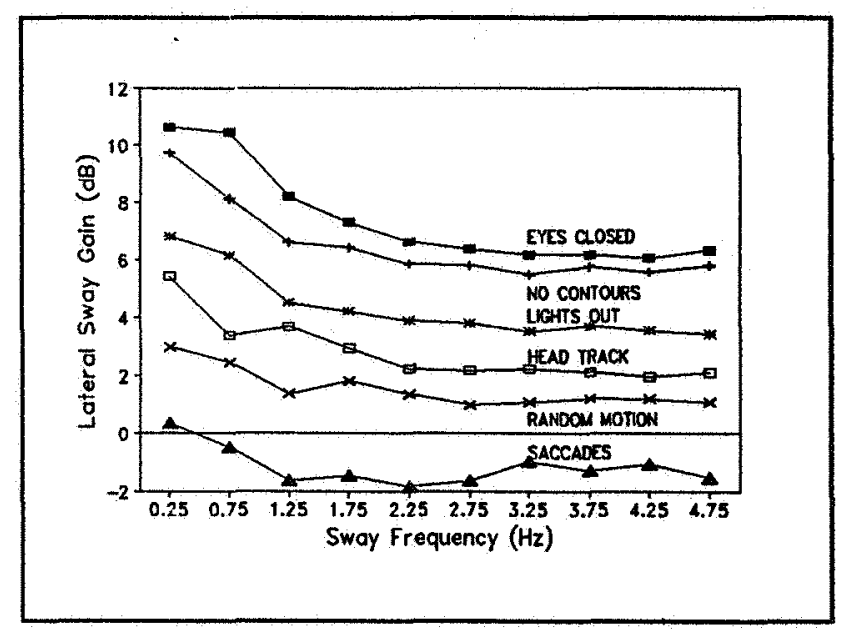

Figure 3. Comparisons of the gain in lateral sway as a function of sway frequency, for experimental and control condition.

subjects made directed saccadic eye movements across the still surroundings. Two of the curves on Figure 3 were moved vertically to reduce overlap: the "lights out" curve was moved down 2 dB and the "head track" curve was moved down $3 \mathrm{~dB}$. "Eyes closed" caused significantly larger sways than either "no contours" or "lights out," which did not differ reliably. "Head track" resulted in significantly smaller sways than in those conditions.

All six curves show a general trend of decreasing gain with increasing frequency, particularly so below $2 \mathrm{~Hz}$. To a frrst approximation, the gain profile associated with any of these six conditions appears nearly interchangeable with the profile from another condition by simply shifting the curve up or down on the graph. Since all of these conditions might serve to reduce or degrade visual feedback from the environment, this first approximation similarity is not overly surprising. In detail, however, it is interesting to note that the saccades condition (6) actually made the subjects sway less than did fixating in still surroundings. Also note that the head track condition (4) is the only curve with a large gain decrease at $0.75 \mathrm{~Hz}$ (midpoint for the 0.5 to $1.0 \mathrm{~Hz}$ band). The frequency dependence of gain profiles with head tracking is more apparent in the following graphs.

Figures 4 and 5 compare the effects of four different gain factors for head tracking. The gain factor parameter relates azimuth positioning of the visible surround to the combined fore-aft and leftright translations plus yaw angle sensed for the head. Each point on these figures represents the average movement gain across 33 subjects, calculated within $0.5 \mathrm{~Hz}$ bandwidths. These gains, the ratios of PSD profiles in tracking vs. baseline conditions, were referenced to "random motion" baseline measures with corresponding gain factors. These baselines were necessary because the visible surround exhibited a slight random jitter due to system noise even when attempting to track a fixed artificial head.

There are two noteworthy features shown in Figures 4 and 5. First, the gain profiles are clearly not flat but instead rise or fall in a frequency-dependent manner. Thus, we must conclude that moving the visual stimulus by tracking the subject's head differs qualitatively from the various other forms of altered visual feedback depicted in Figure 3, because those manipulations have yielded 


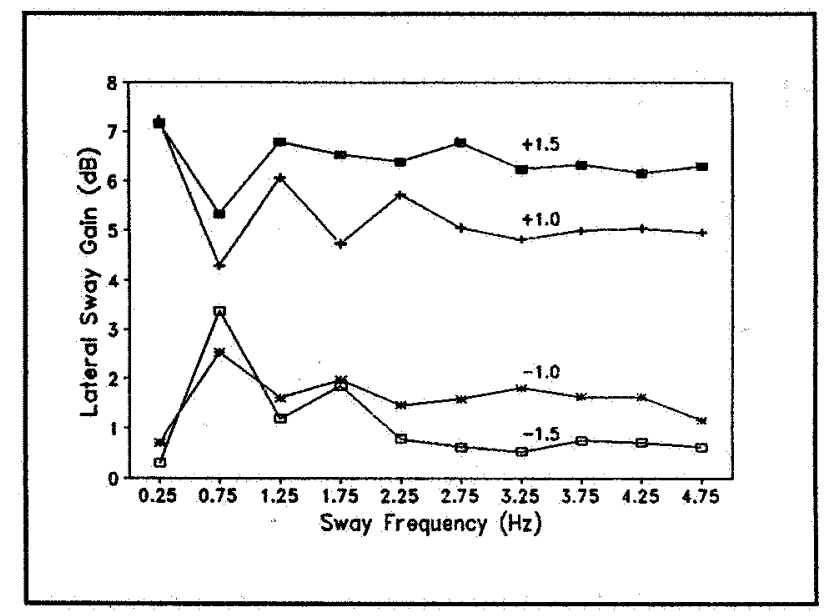

Figure 4. Lateral sway gain as a function of frequency when the azimuth of the surrounds tracked the subject's head movements. The four curves represent different gain factor.

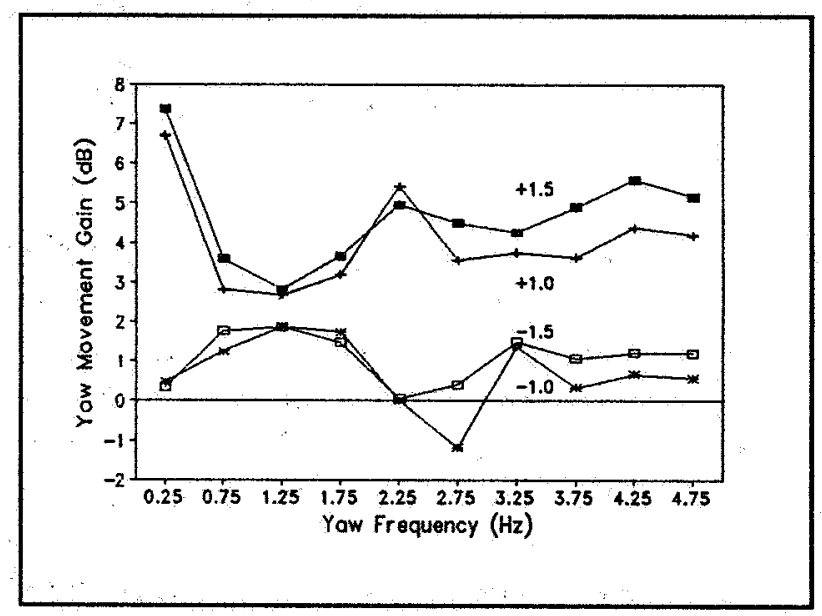

Figure 5. Yaw movement gain as a function of frequency, analogous to Figure 4.

results that are highly similar across frequency. Second, whether each curve in Figures 4 and 5 rises or falls in each frequency band is highly dependent on the sign of the gain factor parameter. For both lateral sway (Fig. 4) and yaw movements (Fig. 5), a gain peak associated with positive parameter values becomes a gain trough when associated with negative parameter values, and vice versa.

Even though subjects in the head tracking experiment showed greater stability than did subjects who simply closed their eyes, 10 out of the 33 reported symptoms of motion sickness. For 4 of those subjects, symptoms persisted more than an hour, and for one subject, persisted 5 hours. Another 8 subjects found the participation unpleasant but not nauseogenic. In contrast, 3 subjects reported extreme exhilaration apparently akin to taking an amusement park ride. Only one subject guessed 
that the stimulus tracked her head movements, while another subject guessed that her head had been captured by motion of the visual stimulus.

\section{CONCLUSIONS}

We find that exposure to a visual virtual environment enslaved to simulate a subject's own eye movements or head movements can have deleterious effects on the subject's spatial stability. When the entire visible surroundings were moved with the same parameters of angular velocity and motion duration which characterize natural saccadic eye movements, subjects tended to lose lateral stability (Fig. 1) and to make head yaw movements (Fig. 2, untriggered motions). This pattern of response was cancelled, however, when saccade-like stimulus motions were made to coincide with the subject's own voluntary eye movements (also in Fig. 2, triggered by saccade). It seems plausible to interpret these findings in the light if theoretical frameworks which propose that a subject generates a hypothetical internal signal regarding their own voluntary eye movements. Such an internal signal (corollary discharge or efference copy) would presumably serve in the natural world to mitigate the effects of retinal image motion accompanying one's voluntary eye movement. In the present virtual world, the same hypothetical internal signal presumably mitigates the effects of external stimulus motion if it is made at the same time when this internal signal is active.

The effects on a subject's spatial stability when the visual surroundings tracked head movements are less readily explained. First, any subject intentions to move the head were discouraged, inasmuch as (a) they were instructed to stand still and (b) they became significantly at risk of falling if they moved the head voluntarily. It must be questioned whether an efference copy or corollary discharge internal signal could exist without subjects' internal plans to make voluntary movements. Second, subjects' movement gain profiles in Figures 4 and 5 are reminiscent of the changes in poles and zeros in the complex domain when a device receiving feedback undergoes a change in its feedback's sign. It is tempting to speculate that head tracking conditions effectively reveal some of the subjects' own internal feedback mechanisms. Unfortunately, the lag time required to update stimulus azimuth after sensing head position and orientation $(150 \mathrm{msec})$ was sufficient to complicate detailed interpretations along these lines. Third, it may be important to understand why so many subjects in the head tracking experiments experienced motion sickness symptoms or other discomforts, even though they did not become very greatly disoriented. We conjecture that the head-tracking visual feedback must have been in conflict with the subjects' own internal models of their orientation in space. Such models are probably multisensory, though highly dependent on visual feedback, and probably established below the level of one's conscious awareness.

We believe that the design of virtual environments which are enslaved to the eye or head movements of a teleoperator must take into account the effects on the human. In the present experiments, we found that these effects can include loss of stability, motion sickness, or other undesirable influences on situational awareness. These presumably could be mitigated by carefully matching sensor resolutions and real time controls of the virtual environment to the capacities of the human participants. 

Poster Presentations Withdrawn:

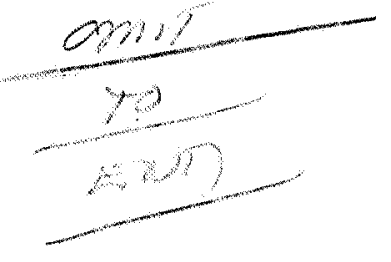

\title{
DESIGN WORK STATION FOR TELEOPERATION TASK ANALYSIS
}

\author{
K. Corker \\ Bolt, Beranek, and Newman, Inc. \\ Cambridge, Massachusetts
}

\section{SUPER AUDITORY LOCALIZATION FOR HUMAN MACHINE INTERFACES}

\author{
N. I. Durlach \\ Massachusetts Institute of Technology \\ Cambridge, Massachusetts
}

\section{CURRENT RESEARCH ON COCKPIT APPLICATIONS OF 3-D AUDITORY DISPLAYS}

\author{
K. O'Donnel and W. T. Melton \\ NASA Ames Research Center \\ Moffett Field, California
}





\section{LIST OF PARTICIPANTS}

Bernard Adelstein*

937 Creston Road

Berkeley, California 94708

Tel-415-524-9129

Harold L. Alexander

Dept. of Aero. and Astro.

Room 33-119

Massachusetts Institute of Technology

Cambridge, Massachusetts 02139 .

Tel-617-258-8799

E-Mail-alex@athena.mit.edu

Haruo Amari**

Researcher

Tokyo Electric Powe Co.

4-10, Irifune, 1-Chome, Chuo-Ku

Tokyo, 104, Japan

Tel-81-3-501-8111, Ext. 6870

Fax-81-3-297-1647

(Co-author with Hirose)

Takashi Aoki

Massachusetts Institute of Technology

Cambridge, Massachusetts 02139

Walter A. Aviles

Naval Ocen Sytems Center

Code 531

P.O. Box 997

Kailua, Hawaii 96734-0997

Tel-808-257-1666

E-Mail-aviles@humu.nosc.mil

Fax-808-257-5231
Ruzena Bajcsy

Dept. of Computer and Information Science

Moore Building

University of Pennsylvania

200 South 33rd Street

Philadelphia, Pennsylvania 19104-6389

Tel-215-898-6222

E-Mail-bajcsy@cis.upenn.edu

Antal K. Bejczy

Jet Propulsion Laboratory

4800 Oak Grove Drive

Pasendena, California 91109

Tel-818-351-9742

C. Thomas Bennett*

Research Psychologist

MS 239-5

NASA Ames Research Center

Moffett Field, California 94035-1000

Tel-415-604-5906

Massimo Bergamasco*

Scuola Superiore S. Anna

Via Carducci, 40

Pisa, I

56100 Italy

K. B. Biggers

Center of Engineering Design

Merrill Engineering Bldg., Room 3176

University of Utah

Salt Lake City, Utah 84112

Jens Blauert

Ruhr-Universitaet Bochum

POB 102148

Bochum, West Germany

Tel-49 234-700-2496

Fax 49 234-700-5568

Telex 0825860 
Frederick P. Brooks

Dept. of Computer Science

CB-3175, Sitterson Hall

University of North Carolina

Chapel Hill, North Carolina 27599

Tel-919-962-1930

E-Mail-brooks@cs.unc.edu

George C. Burdee

Rutgers University

New Jersey

Steve Charles

Center for Engineering Applications

6401 Poplar Avenue/Suite 190

Memphis, Tennessee 38119

Tel-901-767-6662

Francis J. Clark

Dept. of Physiology

College of Medicine

University of Nebraska

Omaha, Nebraska 68105

Tel-402-559-6478

E-Mail-fclark\%unmcvm.bitnet

@umrvmb.umr.edu

Fax-402-559-4438

H. Steve Colburn

Dept. of Biomedical Engineering

Boston University

44 Cummington Street

Boston, MassachusettsA 02215

Tel-617-353-4342

E-Mail-colburn@buenga.bu.edu

C. C. Davis

Center ofr Engineering Design

Merrill Engineering Bldg., Room 3176

Universtiy of Utah

Salt Lake City, Utah 84112
Steven V. DeGennaro

Continuous Speech Recognition Group

Computer Sciences Department

T. J. Watson Research Center

P.O. Box 218

Yorktown Heights, New York 10598

Tel-914-945-3684 or 3638

E-Mail-degenn@IBM.com

Nathaniel I. Durlach

Sensory Communication Group

Room 36-709

Research Laboratory of Electronics

Massachusetts Institute of Technology

Cambridge, Massachusetts 02139

Tel-617-253-2534

Fax-617-258-7864

E-Mail-durlach@cbgrle.mit.edu

Stephen R. Ellis

Aerospace Human Factors Division

MS 239-3

NASA Ames Research Center

Moffett Field, California 94305-1000

Tel-415-604-6147 or 5240

E-Mail-silly@eos.arc.nasa.gov

Mica Endsley*

Northrup Corporation

25900 Oak Street, \#123

Lomita, California 90717

Tel-213-332-0817

Thomas Etter

ANPA West

25 Buena Vista

Mill Valley, California 94941

Tel-415-388-4820

Scott S. Fisher

11571 Buena Vista Dr

Los Altos Hills, California 94022

(415) 941-2272 
Woodie Flowers**

Dept. of Mechanical Engineering

Room 3-461A

Massachusetts Institute of Technology

Cambridge, Massachusetts 02139

(Co-author with Russo)

Thomas A. Furness

Dept. of Industrial Engineering

Mail Code FU-20

University of Washington

Seattle, Washington 98195

Tel-206-543-4600 or 4608

E-Mail-tfurness@blake.acs.washington.edu

Kaigham J. Gabriel

Institute of Industrial Science

University of Tokyo

7-22-1 Roppongi, Minato-ku

Tokyo 106, Japan

Tel-(81) (3) 402-6231

E-Mail-Ken@tkl.iis.u-tokyo.ac.jp

S. K. Ganapathy

Machine Perception Laboratory

4E-622

AT\&T Bell Laboratories

Holmdel, New Jersey 07733

Tel-201-949-7860

Fax-201-949-0399

E-Mail-skg@vax135i.att.com

Julia E. Greenberg

Massachusetts Institute of Technology

Cambridge, Massachusetts 02139

Blake Hannaford

Dept. of Electrical Engineering

Mail Code FT-10

University of Washington

Seattle, Washington 98195

Tel-206-543-2197

E-Mail-blake@blake.acs.washington.edu
Richard M. Held

Dept. of Brain and Cognitive Sciences

Room E10-145B

Massachusettes Institute of Technology

Cambridge, Massachusetts 02139

Tel-617-253-5745

E-Mail-held@psyche.mit.edu

Michitaka Hirose*

University of Tokyo

Currently visiting Prof. Stark

School of Optometry

U.C. Berkeley

Berkeley, California 94720

E-Mail-hirose@opus.berkeley.edu

Neville Hogan

Dept. of Mechanical Engineering

Room 3-449D

Massachusetts Institute of Technology

Cambridge, Massachusetts 02139

Tel-617-253-2277

E-Mail-neville@athena.mit.edu

John M. Hollerbach

Biomedical Engineering Department

McGill University

3775 University Street

Montreal PQ

Canada H3A 2B4

Tel-514-398-8080

Ian W. Hunter

Biomedical Engineering Department

McGill University

3775 University Street

Montreal PQ

Canada H3A 2B4

Tel-514-398-6740 
Professor Gideon Inbar*

Dept of Electrical Engineering

Technion - Israel Institute of Technology

Haifa, Israel 32000

Tel-972-4-293240

Fax-011972-4-221581

E-Mail-inbar@techsel.bitnet

E. K. Iversen

Center for Engineering Design

Merrill Engineering Bldg., Room 3176

University of Utah

Salt Lake City, Utah 84112

Stephen C. Jacobsen

Center for Engineering Design

Merrill Engineering Bldg., Room 3176

University of Utah

Salt Lake City, Utah 84112

Tel-801-581-6499

E-Mail-jacobsen@ced.utah.edu

Fax 801-581-5304

Heidi Jacobus*

President

Charles Systems Corp.

820 Heatherway

Ann Arbor, MI 48104

Tel-313-668-2567

Fax-313-668-8780

E-Mail-Heidi-Jacobus@um.cc.umich.edu

Demetrius G. Jalatis

Central Research Lab

Red Wing, MN

Henry Jex

Systems Technology, Inc.

13766 South Hawthorne Blvd.

Hawthorne, California 90250

Tel-213-679-2281
Kenneth O. Johnson

Dept. of Neuroscience

School of Medicine

Johns Hopkins University

725 North Wolfe St.

Baltimore, Maryland 21205

Tel-301-955-2730

E-Mail-kenneth.johnson@bard.med.jhu.edu

Lynette A. Jones

Physical and Occupational Therapy

Faculty of Medicine

McGill University

3654 Drummond Street

Montreal, PQ

Canada H3G 1 Y5

Tel-514-398-4499 or 4455

E-Mail-lyn @palvax.medcor.mcgill.ca

Seong K. Kang

Northwestern Unversity

Evanston, Illinois

H. Kazerooni*

Mechanical Engineering Dept.

111 Church Street S.E.

University of Minnesota

Minneapolis, MN 55455

Tel-612-625-2398

Fax-612-625-3424

Myron Krueger

Artificial Reality Corp.

Box 786

Vernon, Connecticut 06066

Tel-203-871-1375

James R. Lackner

Graybiel Laboratory

Brandeis University

Waltham, Massachusetts 02254-9110

Tel-617-736-2030

Lois Ann Kuntt

University of Florida 
R. H. LaMotte**

Department of Anesthesiology

Yale University School of Medicine

New Haven, Connecticut

(Co-author with Srinivasan)

Jaron Lanier

VPL Research

656 Bair Island Rd/Suite 304

Redwood City, California 94063

Tel-415-361-1710

Fax-415-361-1845

Larry Leifer

Department of Mechanical Engineering

Stanford University

831 Esplanada Way

Stanford, California 94305

Tel-415-723-1869

Tel-415-493-6481

E-Mail-leifer@sunrise.stanford.edu

Daniel T. Ling

IBM T. J. Watson Research Center

Room H2-B64

P.O. Box 704

Yorktown Heights, New York 10598

Tel-914-784-7030

Fax-914-784-7455

E-Mail-dtling@ibm.com

Jack Loomis

Department of Psychology

University of California

Santa Barbara, California 93106

Tel-805-961-2475

Beth A. Marcus

EXOS, Inc.

78 Woburn Street

Lexington, Massachusetts 02173

Tel-617-862-4848

Tel-617-229-2075
Michael McGreevy

MS 262-2 (Room 109)

NASA Ames Research Center

Moffett Field, California 94035-1000

Tel-415-604-5784

E-Mail-mcgreevey@eos.arc.nasa.gov

William T. Melton**

San Jose State University

San Jose, California

(Co-author with O'Donnell)

Paul A. Millman

Northwestern University

Evanston, Illinois

Marvin L. Minsky

Dept. of Electrical Engineering and Computer

Science

Room NE43-820

Massachusetts Institute of Technology

Cambridge, Massachusetts 02139

Tel-617-253-5864

E-Mail-minsky@ai.mit.edu

Keisuke Miyata

University of California at Berkeley

Berkeley, California

Steven A. Murray

Naval Ocean Systems Center

San Diego, California

Mahmood Nahvi*

E1/EE Department

Cal. Polytechnic Institute

San Luis Obispo, California 93407

Tel-805-756-2308

Fax-805-756-6503

Kenneth E. Nemira

NASA Ames Research Center

Moffett Field, California 94035-1000 
Kathleen O'Donnell*

Engineering Research Psychologist .

MS 239-5

NASA Ames Research Center/U.S. Army

Moffett Field, California 94035-1000

Tel-415-604-6249

Fax-415-604-3729

Xiao-Dong Pang**

Sensory Communication Group

Room 36-759

Research Laboratory of Electronics

Massachusetts Institute of Technology

Cambridge, Massachusetts 02139

Misha Pavel

Dept. of Psychology

Bldg. 420

Stanford, California 94305

Tel-415-725-2430

E-Mail-mis@psych.stanford.edu

Ross L. Pepper

Science Applications International, Inc.

41 Hitchcock Way

Santa Barbara, California 93105

Tel-805-569-5549

Tel-805-546-8761

William Rabinowitz

Massachusetts Institute of Technology

Cambridge, Massachusetts 02139

Howard L. Rheingold

306 Poplar

Mill Valley, California

94941

415 388-3912

hlr@well.sf.ca.us

Catherine H. Richards

41 Delaware Ave.

Ottawa Ont

CANADA K2P OZ2

6132338316
Alan Riggs**

Charles Systems Corp

820 Heatherway

Ann Arbor, Michigan 48104

(Co-author with Jacobus)

Michael Rosen**

Dept. of Mechanical Engineering

Room 3-137D

Massachusetts Institute of Technology

Cambridge, Massachusetts 02139

(Co-author with Adelstein)

Massimo Russo

Media Laboratory

Room E15-054

Massachusetts Institute of Technology

Cambridge, Massachusetts 02139

Tel-617-253-0664

E-Mail-marusso@athena.mit.edu

Hagen Schempf

Woods Hole Oceanographic Institute

Woods Hole, Massachusetts 02543

Martin Schwirzke**

NASA Ames Research Center

Moffett Field, California 94035-1000

(Co-author with Bennett)

Thomas B. Sheridan

Dept. of Mechanical Engineering

Room 3-346

Massachusetts Institute of Technology

Cambridge, Massachusetts 02139

Tel-617-253-2228

Carl E. Sherrick

Department of Psychology

Green Hall

Princeton University

Princeton, New Jersey 08544

Tel-609-452-5277 
Kuman V. Siva

AEA Harwell Lab

United Kingdom

Thomas Speeter

AT\&T Bell Laboratories

Rm. 4E-638

Crawfords Corner Road

Holmdel, New Jersey 07733

Tel-201-949-9014

M. A. Srinivasan

Sensory Communication Group

Room 36-709

Research Laboratory of Electronics

Massachusetts Institute of Technolocy

Cambridge, Massachusetts 02139

Lawrence Stark

Telerobotics Unit

481 Minor Hall (OPT)

University of California Berkeley

Berkeley, California 94720

Tel-415-642-7196 or 3621

Robert Stone*

Advanced Robotics Centre

University Road

Salford, Manchester

United Kingdom M5 4PP

Tel-(61)-745-7384

Fax-(61)-745-8264

Alfred Tadros**

Media Laboratory

Massachusetts Institute of Technology

Cambridge, Massachusetts 02139

(Co-author with Russo)

James S. Tittle**

University of California, Irvine

Irvine, California

(Co-author with Bennett)
Susumu Tachi

Biorobotics Division

Mechanical Engineering Lab., MITI

1-2 Namiki, Tsukuba Science City

Ibaraki 305 JAPAN

Tel-81-298-54-2680

E-Mail-m1730@mel.go.jp

Fax-81-298-54-2518

Hong Tan**

Sensory Communication Group

Room 36-359

Research Laboratory of Electronics

Massachusetts Institute of Technology

(Co-author with Xiao-Dong Pang)

Heinz Unbehauen*

Ruhr-Universitaet Bochum

POB 102148

Bochum, West Germany

Tel-49 234-700-4071

Fax-49 234-700-5568

Telex-0825860

William L. Verplank

ID Two

San Francisco, California

Robert B. Welch

Adaptation

NASA Ames Research Center

Mail Stop 239-7

Moffett Field, California 94035-1000

Tel-415-604-5749

Elizabeth M. Wenzel

Mail Stop 262-2

NASA Ames Research Center

Moffett Field, California 94035-1000

Tel-415-604-6290

E-Mail-beth@eos.arc.nasa.gov

Keith D. White

University of Florida 
Dana R. Yoerger

Woods Hole Oceanographic Institution

Deep Submergence Laboratory

Blake 203

Woods Hole, Massachusetts 02543

Tel-508-548-1400 (Ext. 2608 or 2851)

Albert S. Yen

Jet Propulsion Laboratory

California Institute of Technology

4800 Oak Grove Drive

Pasadena, California 91109

Laurence R. Young

Dept. of Aeronautics and Astronautics Room 37-219

Massachusetts Institute of Technology

Cambridge, Massachusetts 02139

Tel-617-253-7759

E-Mail-lry@space.mit.edu
Greg L. Zacharias

Charles River Analytics, Inc.

55 Wheeler Street

Cambridge, Massachusetts 02139

Tel-617-491-3474

David Zeltzer

Media Laboratory

Room E15-324

Massachusetts Institute of Technology

Cambridge, Massachusetts 02139

Tel-617-253-5995

E-Mail-dz@media-lab.media.mit.edu

* Presenting posters

** Co-authors of posters 


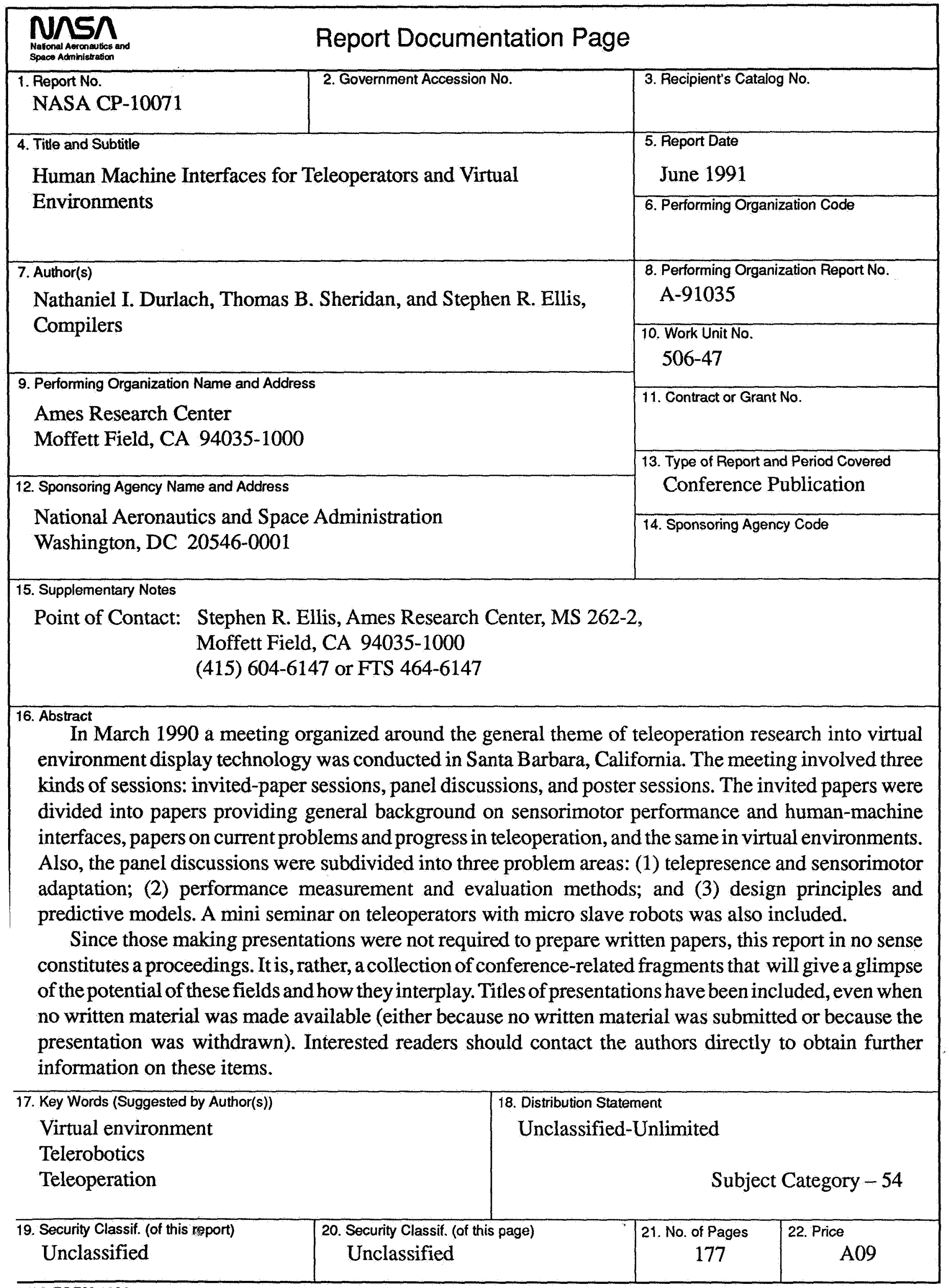

